\title{
Shot noise and spin-orbit coherent control of entangled and spin-polarized electrons
}

\author{
J. Carlos Egues, ${ }^{1,2}$ Guido Burkard, ${ }^{1}$ D. S. Saraga, ${ }^{1}$ John Schliemann, ${ }^{1}$ and Daniel Loss ${ }^{1}$ \\ ${ }^{1}$ Department of Physics and Astronomy, University of Basel, Klingelbergstrasse 82, CH-4056 Basel, Switzerland \\ ${ }^{2}$ Departamento de Física e Informática, Instituto de Física de São Carlos, Universidade de São Paulo, 13560-970 São Carlos, \\ São Paulo, Brazil
}

(Received 4 September 2005; published 20 December 2005)

\begin{abstract}
We extend our previous work on shot noise for entangled and spin polarized electrons in a beam-splitter geometry with spin-orbit (SO) interaction in one of the incoming leads (lead 1). In addition to accounting for both the Dresselhaus and the Rashba spin-orbit terms, we present general formulas for the shot noise of singlet and triplets states derived within the scattering approach. We determine the full scattering matrix of the system for the case of leads with two orbital channels coupled via weak SO interactions inducing channel anticrossings. We show that this interband coupling coherently transfers electrons between the channels and gives rise to an additional modulation angle-dependent on both the Rashba and Dresselhaus interaction strengthswhich allows for further independent coherent control of the electrons traversing the incoming leads. We derive explicit shot noise formulas for a variety of correlated pairs (e.g., Bell states) and lead spin polarizations. Interestingly, the singlet and each of the triplets defined along the quantization axis perpendicular to lead 1 (with the local SO interaction) and in the plane of the beam splitter display distinctive shot noise for injection energies near the channel anticrossings; hence, one can tell apart all the triplets, in addition to the singlet, through noise measurements. We also find that spin-orbit induced backscattering within lead 1 reduces the visibility of the noise oscillations, due to the additional partition noise in this lead. Finally, we consider injection of two-particle wavepackets into leads with multiple discrete states and find that two-particle entanglement can still be observed via noise bunching and antibunching.
\end{abstract}

DOI: 10.1103/PhysRevB.72.235326 PACS number(s): 73.23. - b, 71.70.Ej, 72.70. $+\mathrm{m}$, 72.25. $-\mathrm{b}$

\section{INTRODUCTION}

Spin-related effects underlie promising possibilities in the emerging field of semiconductor spintronics and spin-based quantum computing. ${ }^{1,2}$ Spin-entangled electron pairs in unconventional geometries, e.g., electron beam splitters, ${ }^{3}$ offer a unique setting in which to investigate fundamental nonlocal electron correlations in solids. ${ }^{4}$ Several schemes for creating and injecting entangled pairs in mesoscopic systems have recently been proposed involving quantum dots, superconductors, and interference in the electron flow. ${ }^{5-25}$ Detection, coherent manipulation, and transfer of spin entanglement ("flying qubits") in nanostructures are crucial ingredients for quantum-information processing and communication. Nonequilibrium noise, shot noise, is a useful probe for detecting entanglement. ${ }^{6,26}$

More recently, the Rashba spin-orbit interaction present in confined electron systems lacking structural inversion symmetry ${ }^{27}$ has been proposed as a convenient means to spin rotate entangled pairs. ${ }^{28}$ Interestingly, it was found that a local Rashba spin-orbit interaction acting upon a nonlocal portion of spatially separated entangled electron pairs injected into a beam splitter gives rise to sizable modulation of the shot noise in the outgoing leads. ${ }^{28}$ The use of the Rashba interaction to controllably rotate the electron spin was first proposed by Datta and Das. ${ }^{29}$ Motivated by this earlier proposal and its potential impact on semiconductor spintronics, many researchers are actively investigating spin-orbitrelated physics in a variety of semiconductor nanostructures. ${ }^{30,31,34-45}$

Here we extend our previous investigation on the coherent SO control of entangled and spin-polarized electrons and their shot noise for transport in a beam-splitter configuration (Fig. 1) with local spin-orbit interactions, i.e., interactions acting within only a finite region of one of the two onedimensional incoming leads. ${ }^{28}$ We include both the Rashba ${ }^{27}$ and the Dresselhaus ${ }^{46}$ spin-orbit terms. ${ }^{47}$ Since the Rashba part of the SO coupling is gate tunable, ${ }^{48}$ one can controllably spin rotate the incoming correlated spinor pairs thus changing the degree of symmetry of the spin part of pair wave function. The stringent requirement of antisymmetry for fermions-the Pauli principle-intrinsically links the spin and the orbital (charge) degrees of freedom. ${ }^{5}$ Thus the spin-orbit induced spin rotation affects the spatial charge distribution of the pair which can be probed via currentfluctuation measurements: charge shot noise.

We consider a beam splitter with quasi-one-dimensional incoming leads with one and two channels. (i) For singlemoded leads and within the scattering approach we generalize our previous results ${ }^{28}$ by deriving general expressions for the shot noise of singlet and triplet pairs injected into the beam splitter. We present explicit formulas for the particular beam-splitter scattering matrix of the experiment in Ref. 3 and a variety of incoming electron pairs: singlet and entangled and unentangled triplet states defined along distinct quantization axes. (ii) The case with two channels is particularly interesting as the SO terms give rise to interchannel coupling which results in anticrossings of the bands. For incoming energies near these avoided crossings, we find similarly to Ref. 28 an additional spin phase due to the coherent transfer of carriers between the SO coupled bands. Here, however, this modulation angle depends on both the Rashba and the Dresselhaus coupling strengths. Interestingly, for singlet and triplets defined along the $y$ quantization axis (Fig. 1) 


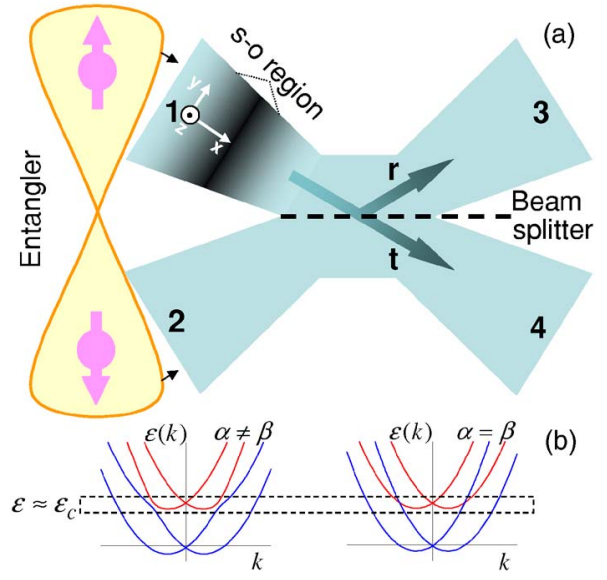

FIG. 1. (Color online) (a) Spin-entangled electrons injected into a beam-splitter setup with spin-orbit interactions, Rashba and Dresselhaus, within a finite region $L$ of lead 1. The strength $\alpha$ of the Rashba interaction can, in principle, be controlled via a top gate so as to be equal or unequal to the Dresselhaus coupling $\beta$. For two orbital channels in lead 1 and $\alpha=\beta$, no SO-induced band mixing occurs, right panel (b). For $\alpha \neq \beta$ (or when either $\alpha=0$ or $\beta=0$ ) the bands anti cross, left panel (b). Only a single spin rotation $\theta_{\mathrm{SO}}$ $=2 m \sqrt{\alpha^{2}+\beta^{2}} L / \hbar^{2}$ is present for $\alpha=\beta$, while an additional "mixing" spin rotation $\theta_{d}$ modulates the electron transport in lead 1 for $\alpha$ $\neq \beta$ and impinging energies near the crossing $\varepsilon \approx \varepsilon_{c}$. This modulation appears in the current fluctuations (shot noise) measured in lead 3. In particular, each of the triplets - for a quantization axis along the $y$ direction-exhibits a distinctive noise as a function of $\left(\theta_{\mathrm{SO}}, \theta_{d}\right)$.

and injected into only one of the two channels of the incoming leads, we find that each of the triplet (besides the singlet) pairs displays distinctive noise modulations. This provides a way of distinguishing all of these triplet pairs via noise measurements. The interband coupling controlling the extra phase can, in principle, be varied via independent side gates which change the width of the incoming channels ${ }^{28}$ this provides an additional mechanism for electric spin control. Moreover, for tuned SO couplings (i.e., equal strengths) the Rashba and Dresselhaus terms partially cancel themselves out, thus giving rise to parabolic-band crossings for arbitrary strength of the SO interaction, ${ }^{49}$ Fig. 1(b). This allows for the propagation of electron spins protected against nonmagnetic scattering, i.e., robust entangled or unentangled spin pairs.

We also consider spin-polarized injection ${ }^{50,51}$ into the beamsplitter. Here we find that noise measurement can probe the spin polarization of the Fermi-liquid leads along distinct quantization directions. We also discuss the effects of backscattering in the incoming leads, due to, e.g., the potential discontinuities at the entrance and exit of the SO active region in lead 1 (see Appendix B for an explicit evaluation of the transmission coefficient for electrons crossing a 1D lead with SO interaction). Backscattering reduces the visibility of the shot noise oscillations, because of the additional partition noise in the incoming leads. Finally, we investigate transport of injected two-particle wave packets into leads with multiple discrete states but without SO interaction. Similarly to our previous results ${ }^{6}$ with ordinary two-particle pairs (i.e., "plane waves"), we find that two-particle entanglement can also be detected via noise measurements (bunching and antibunching) even with incoming wave packets.

This paper is organized as follows. In Sec. II we introduce the spin-orbit Hamiltonian in one-dimensional (1D) channels. We consider both the Rashba and the Dresselhaus SO terms. We present exact and approximate solutions for wires with, respectively, equal and unequal (Rashba and Dresselhaus) SO coupling strengths. The full SO transfer matrix for wires with one and two (coupled) channels is also derived. The boundary conditions for the two coupled channel case are discussed in detail. In Sec. III we present the basics of the scattering formalism for current and shot noise of spinentangled electron pairs and spin-polarized electrons. We derive general formulas for the shot noise of singlet and triplet pairs injected into a beam splitter with an arbitrary scattering matrix (Sec. III B). The effect of backscattering is also discussed (Sec. III C) for electron pairs in single-moded incoming leads. We present many specific formulas for the noise of Bell pairs, electron pairs defined along distinct quantization axes for both single- and double-moded wires. Noise for spin-polarized injection is discussed in (Sec. III D). We also consider (Sec. III E) the injection of entangled and unentangled wavepackets into leads with multiple energy levels. We summarize our results and conclusions in Sec. IV. Many technical details of our calculation are discussed in the Appendixes A-E.

\section{SPIN-ORBIT COUPLING IN 1D CHANNELS: RASHBA AND DRESSELHAUS}

Quantum wires can be defined from two-dimensional electron gases by further constraining the electron motion to one spatial direction via, for instance, gate electrodes. When the underlying 2DEG has spin-orbit interactions of the Dresselhaus $^{46}$ and Rashba ${ }^{27}$ types, due to bulk inversion asymmetry (BIA) and structural inversion asymmetry (SIA), respectively, the 1D channel so formed will also present such interaction terms. ${ }^{52}$ The Hamiltonian of a $2 D E G$ with spin orbit interaction and an additional gate-induced confining potential $V(y)$ reads

$$
\begin{aligned}
H= & -\frac{\hbar^{2}}{2 m}\left(\frac{\partial^{2}}{\partial x^{2}}+\frac{\partial^{2}}{\partial y^{2}}\right)+V(y) \\
& +i \alpha\left(\sigma_{y} \partial_{x}-\sigma_{x} \partial_{y}\right)+i \beta\left(\sigma_{y} \partial_{y}-\sigma_{x} \partial_{x}\right),
\end{aligned}
$$

where $\partial_{i} \equiv \partial / \partial i, i=x, y$ and the third and fourth terms are the usual Rashba (strength $\alpha$ ) and the linearized Dresselhaus (strength $\beta$ ) SO terms, respectively.

\section{A. Exact solution: $\alpha=\beta$ case}

Similarly to the two-dimensional case treated in Ref. 49, the SO wire problem here is exactly solvable for tuned couplings $|\alpha|=\beta$. Let us first consider the general case of a twodimensional electron gas with an arbitrary scalar potential $V(\vec{r})$ which can, e.g, describe static nonmagnetic impurities, or further confinements creating a quantum wire or a quantum dot. At the symmetry points $\alpha= \pm \beta$ the operator $\Sigma$ $=\left(\sigma^{x} \mp \sigma^{y}\right) / \sqrt{2}$ provides an additional conserved quantity, 
and a general eigenstate of $\mathcal{H}$ and $\Sigma$ reads (for $\alpha=-\beta$ )

$$
\psi_{ \pm}(\vec{r})=\frac{1}{\sqrt{2}}\left(\begin{array}{c}
1 \\
\pm e^{i \pi / 4}
\end{array}\right) \varphi(\vec{r}) e^{\mp i \sqrt{2} \alpha m(x-y) / \hbar^{2}}
$$

in the $\sigma_{z}$ basis. The function $\varphi(\vec{r})$ fulfills the usual spinindependent Schrödinger equation

$$
\left(-\frac{\hbar^{2}}{2 m} \nabla^{2}+V(\vec{r})\right) \varphi(\vec{r})=\left(\varepsilon+\frac{2 \alpha^{2} m}{\hbar^{2}}\right) \varphi(\vec{r}),
$$

and $\varepsilon$ is the energy eigenvalue of the wave function $\psi_{ \pm}(\vec{r})$ with $\Sigma= \pm 1$. Now consider a quantum wire along the $x$ direction, i.e., $V(\vec{r})=V(y)$. At $\alpha=-\beta$ the wave functions are of the form (2) with $\varphi_{n}(\vec{r})=\phi_{n}(y) \exp \left[i\left(k \pm \sqrt{2} \alpha m / \hbar^{2}\right) x\right] / \sqrt{L_{x}}, L_{x}$ is a normalizing length, such that the full wave function reads

$$
\psi_{n, \pm}(\vec{r})=\frac{1}{\sqrt{2}}\left(\begin{array}{c}
1 \\
\pm e^{i \pi / 4}
\end{array}\right) \frac{e^{i k x}}{\sqrt{L_{x}}} \phi_{n}(y) e^{ \pm i \sqrt{2} \alpha m y / \hbar^{2}},
$$

where $\phi_{n}(y)$ obeys the usual Schrödinger equation for the transverse variable $y$ with quantized eigenvalues $\widetilde{\varepsilon}_{n}$. The eigenstates (4) are characterized by the subband index $n$ and the wave number $k$, and the corresponding eigenenergies are given by $\varepsilon_{n}^{ \pm}(k)=\widetilde{\varepsilon}_{n}+\left(\hbar^{2} / 2 m\right)\left(k \pm \sqrt{2} \alpha m / \hbar^{2}\right)^{2}-2 \alpha^{2} m / \hbar^{2}$. Note that, similarly to the two-dimensional case discussed earlier, ${ }^{49}$ the wire energy dispersions here are also parabolic - for any strength of the $|\alpha|=\beta$ coupling, see Fig. $1(b)$.

\section{B. Approximate solutions: $\alpha \neq \beta$ case}

For unequal couplings we first solve the quantum wire problem in the absence of spin orbit coupling and then use this solution as a basis to write down the Hamiltonian matrix with the SO terms. Here we neglect any additional SO terms arising from the further confinement ${ }^{30} V(y)$.

\section{Quantum wire eigenstates}

The solution to Eq. (1) without the SO terms is

$$
\varphi_{k, n, \sigma_{z}}(x, y)=\frac{e^{i k x}}{\sqrt{L_{x}}} \phi_{n}(y)\left|\sigma_{z}\right\rangle,
$$

where $\left|\sigma_{z}\right\rangle \in\left\{|\uparrow\rangle_{z}|\downarrow\rangle_{z}\right\}$ is the electron spin state in the $\sigma_{z}$ basis, with eigenvalues

$$
\varepsilon_{k, n, \sigma_{z}}=\frac{\hbar^{2} k^{2}}{2 m}+\epsilon_{n}
$$

and $n=a, b, \ldots$, denoting the transverse modes with energies $\epsilon_{n}$ (note that $\widetilde{\varepsilon}_{n}=\epsilon_{n}$ in the absence of SO). The transverse confining eigenfunctions $\phi_{n}(y)$ obey the 1D Schödinger equation

$$
-\frac{\hbar^{2}}{2 m} \frac{d^{2} \phi_{n}(y)}{d y^{2}}+V(y) \phi_{n}(y)=\epsilon_{n} \phi_{n}(y) .
$$

The confining potential in Eq. (7) is arbitrary. Later on we consider an explicit form (obtained for hard-wall confinement) so as to obtain simple estimates.

\section{Rashba-Dresselhaus wire}

We can derive a reduced Hamiltonian for our quantum wire with SO by expanding the solution of Eq. (1) in the basis of the wire without $\mathrm{SO},\left\{\varphi_{k, a, \uparrow}, \varphi_{k, a, \downarrow}, \varphi_{k, b, \uparrow}, \varphi_{k, b, \downarrow}\right\}$. Here we consider only two wire modes. We then find

$$
H=\left[\begin{array}{cccc}
\frac{\hbar^{2} k^{2}}{2 m}+\epsilon_{a} & (i \alpha+\beta) k & 0 & (-i \alpha+\beta) d_{a b} \\
(-i \alpha+\beta) k & \frac{\hbar^{2} k^{2}}{2 m}+\epsilon_{a} & (-i \alpha-\beta) d_{a b} & 0 \\
0 & (i \alpha-\beta) d_{a b}^{*} & \frac{\hbar^{2} k^{2}}{2 m}+\epsilon_{b} & (i \alpha+\beta) k \\
(i \alpha+\beta) d_{a b}^{*} & 0 & (-i \alpha+\beta) k & \frac{\hbar^{2} k^{2}}{2 m}+\epsilon_{b}
\end{array}\right]
$$

The matrix element

$$
d_{a b}=-d_{b a}^{*} \equiv\left\langle\phi_{a}|\partial / \partial y| \phi_{b}\right\rangle
$$

in Eq. (8) defines the SO induced interband mixing between the wire modes arising from the SO terms proportional to $p_{y}$ in Eq. (1). For hard-wall confinement $d_{a b}=8 / 3 w$, where $w$ is the wire width. It is convenient to rewrite the above matrix in the basis of the eigenstates corresponding to $d_{a b}=0$. For null interband coupling the Hamiltonian decouples into two sets of SO bands

$$
\varepsilon_{n}^{s}(k)=\frac{\hbar^{2} k^{2}}{2 m}+\epsilon_{n}-s k \sqrt{\alpha^{2}+\beta^{2}},
$$

where $n=a, b$ and $s= \pm$, and eigenvectors 


$$
\varphi_{k, n, s}(x, y)=\frac{e^{i k x}}{\sqrt{L_{x}}} \phi_{n}(y)|s\rangle
$$

with

$$
|s\rangle=\frac{1}{\sqrt{2}}\left(\begin{array}{c}
1 \\
-s \xi
\end{array}\right)=\frac{1}{\sqrt{2}}\left(|\uparrow\rangle_{z}-s \xi|\downarrow\rangle_{z}\right)
$$

where

$$
\bar{H}=\left(\begin{array}{cccc}
\varepsilon_{a}^{+} & 0 & 2 i d_{a b} \frac{\alpha \beta}{\sqrt{\alpha^{2}+\beta^{2}}} & -d_{a b} \frac{\alpha^{2}-\beta^{2}}{\sqrt{\alpha^{2}+\beta^{2}}} \\
0 & \varepsilon_{a}^{-} & d_{a b} \frac{\alpha^{2}-\beta^{2}}{\sqrt{\alpha^{2}+\beta^{2}}} & -2 i d_{a b} \frac{\alpha \beta}{\sqrt{\alpha^{2}+\beta^{2}}} \\
-2 i d_{a b}^{*} \frac{\alpha \beta}{\sqrt{\alpha^{2}+\beta^{2}}} & d_{a b}^{*} \frac{\alpha^{2}-\beta^{2}}{\sqrt{\alpha^{2}+\beta^{2}}} & \varepsilon_{b}^{+} & 0 \\
-d_{a b}^{*} \frac{\alpha^{2}-\beta^{2}}{\sqrt{\alpha^{2}+\beta^{2}}} & 2 i d_{a b}^{*} \frac{\alpha \beta}{\sqrt{\alpha^{2}+\beta^{2}}} & 0 & \varepsilon_{b}^{-}
\end{array}\right) .
$$

The diagonalization of Eq. (15) is straightforward; the eigenenergies are

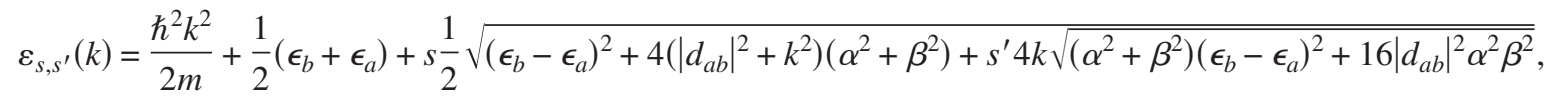

where $s, s^{\prime}= \pm$. The corresponding eigenfunctions are too lengthy to be shown here. Figure 1(b) shows the above energy dispersions for $\alpha \neq \beta$ and $\alpha=\beta$ for nonzero interband coupling $d_{a b}$. In general, the energy dispersions present avoided crossings for $\alpha \neq \beta$. In contrast, the SO tuned $\alpha=\beta$ case has eigenvalues which are quadratic in $k$ with no avoided crossings. This $k$ dependence is easily seen by setting $\alpha=\beta$ in Eq. (16)

$$
\begin{aligned}
\varepsilon_{s, s^{\prime}}(k)= & \frac{\hbar^{2} k^{2}}{2 m}+\frac{1}{2}\left(\epsilon_{b}+\epsilon_{a}\right)+s \sqrt{2} k \alpha \\
& +s^{\prime} \frac{1}{2} \sqrt{\left(\epsilon_{b}-\epsilon_{a}\right)^{2}+8 \alpha^{2}\left|d_{a b}\right|^{2}} .
\end{aligned}
$$

In what follows we discuss in more detail the cases $d_{a b}$ $=0$ and $d_{a b} \neq 0$ corresponding to the uncoupled and interband-coupled channels, respectively. We emphasize again that the interband coupling described by the matrix element $d_{a b}$ is purely induced by the SO. As we will see below, the uncoupled case gives rise to a single spin-rotation modulation. The interband coupled case, on the other hand, will have two independent modulation angles for injected electrons with energies near the band crossings.

\section{Uncoupled 1D channels $\left(d_{a b}=0\right)$ : Single spin rotation $\theta_{R}$}

Here we have in mind a two-terminal geometry with the source and drain connected by a Rashba-Dresselhaus wire. For simplicity, we neglect the band offsets between the various interfaces. That is, we assume a unity transmission through the SO region. ${ }^{53}$ Finite offsets give rise to FabryPerot-type oscillations which further modulate the transport properties $^{31}$ of the system. The uncoupled case $\left(d_{a b}=0\right)$ considered here should be a good approximation also for finite $d_{a b}$, provided that $\alpha\left|d_{a b}\right|$ be much smaller than the interband energy separation $\left(\alpha\left|d_{a b}\right| \ll \epsilon_{b}-\epsilon_{a}\right)$. The solution for $d_{a b}=0$ is straightforward (see Ref. 31 for the case where only the Rashba coupling is active). From Eq. (15), which is diagonal for $d_{a b}=0$, we immediately obtain the two sets of SO bands [Eq. (10)] which we rewrite as

$$
\varepsilon_{a, b}^{(s)}(k)=\frac{\hbar^{2}}{2 m}\left(k-s k_{\mathrm{SO}}\right)^{2}+\epsilon_{a, b}-\frac{\hbar^{2} k_{\mathrm{SO}}^{2}}{2 m}, \quad s= \pm,
$$

where

$$
k_{\mathrm{SO}} \equiv m \sqrt{\alpha^{2}+\beta^{2}} / \hbar^{2}
$$

is the SO wave vector. The corresponding eigenvectors are given in Eqs. (11) and (12). For $d_{a b}=0$ the SO bands cross at 


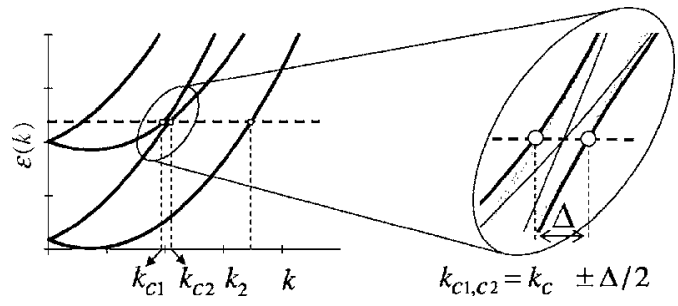

FIG. 2. Schematic of the quantum wire energy dispersions $\varepsilon_{s, s^{\prime}}(k)$ [Eq. (16)] for $\alpha \neq \beta$. The blowup shows the band anticrossing for $d \neq 0$ in more detail. The crossing thin solid lines represent the uncoupled case $d_{a b}=0$. The curves with circles are obtained from Eq. (29) $\left[\varepsilon_{ \pm}(k)\right]$ and are good approximation for the actual dispersions near crossing point $k_{c}^{0}$. The wave vectors $k_{c 1}, k_{c 2}$, and $k_{2}$, used to expand an incoming plane wave within the SO region [Eq. (35)], are also shown in the inset.

$$
k_{c}=\frac{\epsilon_{b}-\epsilon_{a}}{2 \sqrt{\alpha^{2}+\beta^{2}}},
$$

which is obtained by setting $\varepsilon_{a}^{-}\left(k_{c}\right)=\varepsilon_{b}^{+}\left(k_{c}\right)$ (see thin solid line in the inset of Fig. 2); a symmetric crossing also occurs for at $k=-k_{c}$.

As first pointed out by Datta and Das, ${ }^{29}$ injected electrons moving down the 1D channel will spin precess due to the action of the SO interaction. Here the spin rotation is due to the combined effects of the Rashba and Dresselhaus terms. In analogy to the case discussed by Datta and Das, here we find that a spin-up electron, say in channel $a$, crossing the length $L$ of the SO active region will emerge in the state

$$
|\uparrow\rangle_{z} \rightarrow \cos \left(\theta_{\mathrm{SO}} / 2\right)|\uparrow\rangle_{z}-\sin \left(\theta_{\mathrm{SO}} / 2\right)|\downarrow\rangle_{z},
$$

where

$$
\theta_{\mathrm{SO}}=2 m \sqrt{\alpha^{2}+\beta^{2}} L / \hbar^{2}
$$

is the spin rotation angle about the $y$ axis. Similarly, a spin down electron evolves into

$$
|\downarrow\rangle_{z} \rightarrow \sin \left(\theta_{\mathrm{SO}} / 2\right)|\uparrow\rangle_{z}+\cos \left(\theta_{\mathrm{SO}} / 2\right)|\downarrow\rangle_{z} .
$$

The same reasoning applies to impinging electrons in channel $b$. Hence, we can described the SO region in the absence of SO induced channel coupling (uncoupled channels) by the $4 \times 4$ "transfer" matrix $\mathbf{U}_{\text {SO }}^{u}$

$$
\mathbf{U}_{\mathrm{SO}}^{u}=\left(\begin{array}{cc}
\mathbf{U}_{\mathrm{SO}}^{a} & 0 \\
0 & \mathbf{U}_{\mathrm{SO}}^{b}
\end{array}\right)
$$

where

$$
\mathbf{U}_{\mathrm{SO}}^{a}=\mathbf{U}_{\mathrm{SO}}^{b}=\left(\begin{array}{cc}
\cos \left(\theta_{\mathrm{SO}} / 2\right) & \sin \left(\theta_{\mathrm{SO}} / 2\right) \\
-\sin \left(\theta_{\mathrm{SO}} / 2\right) & \cos \left(\theta_{\mathrm{SO}} / 2\right)
\end{array}\right),
$$

defines the single-channel transfer matrix for the uncoupled channels $a$ and $b$. Later on we introduce the scattering matrix approach to calculate current and noise in a beam-splitter geometry. The SO rotation matrix above (and its generalization for two channels) will prove very convenient in accounting for SO effects on the transport properties of the beam splitter within the scattering approach. Note that only the Rashba coupling constant appearing in the rotation angle $\theta_{\mathrm{SO}}$ can be varied externally via a gate electrode, while the Dresselhaus coupling $\beta$ is a material property. As a final point, we note that the above SO rotated states satisfy the proper boundary conditions for the wave function at $x=0$ and $x=L$. This is discussed in some detail in Appendix A for both the one- and two-channel cases.

\section{Coupled $1 D$ channels $\left(d_{a b} \neq 0\right)$ : Additional spin rotation $\theta_{d}$ for $\alpha \neq \beta$}

For nonzero SO induced interband coupling $d_{a b}$, the subbands anticross for distinct coupling strengths $\alpha \neq \beta$. Similarly to the one-channel case, here we also have to find out how incoming spin up (or down) electrons emerge after traversing the $\mathrm{SO}$ active region of length $L$. Here we have in mind incoming electrons with energies near the $d_{a b}=0$ crossing of the bands at $k_{c}$, i.e., $\varepsilon \sim \varepsilon_{a}^{-}\left(k_{c}\right)=\varepsilon_{b}^{+}\left(k_{c}\right)$. This is the relevant energy range where SO induced interband crossing should play a role (unless $\alpha=\beta$ ). In what follows we present a simple analysis of this injection problem by using a perturbative approach ("near free electron model" $" 54$ ) to describe the SO states near the crossings.

For injection energies near the $d_{a b}=0$ crossings, we can approximate the Hamiltonian in Eq. (15) by

$$
H_{\mathrm{app}}=\left(\begin{array}{cccc}
\varepsilon_{a}^{-} & 0 & 0 & 0 \\
0 & \varepsilon_{a}^{+} & d_{a b} \frac{\alpha^{2}-\beta^{2}}{\sqrt{\alpha^{2}+\beta^{2}}} & 0 \\
0 & d_{a b}^{*} \frac{\alpha^{2}-\beta^{2}}{\sqrt{\alpha^{2}+\beta^{2}}} & \varepsilon_{b}^{-} & 0 \\
0 & 0 & 0 & \varepsilon_{b}^{+}
\end{array}\right) \text {, }
$$

i.e., we drop all the off-diagonal matrix elements except those directly coupling the states near the crossing. From the form of $H_{\text {app }}$ it is obvious that the crossing states [middle block of Eq. (26)] will split due to the $d_{a b}$ coupling. The new eigenvalues are

$$
\varepsilon_{ \pm}(k)=\frac{\hbar^{2} k^{2}}{2 m}+\frac{1}{2}\left(\epsilon_{b}+\epsilon_{a}\right) \pm\left|d_{a b}\right| \frac{\alpha^{2}-\beta^{2}}{\sqrt{\alpha^{2}+\beta^{2}}} \sqrt{1+x}
$$

where

$$
x=\frac{\left[\left(\epsilon_{b}-\epsilon_{a}\right)-2 \sqrt{\alpha^{2}+\beta^{2}} k\right]^{2}}{4\left(\left|d_{a b}\right| \frac{\alpha^{2}-\beta^{2}}{\sqrt{\alpha^{2}+\beta^{2}}}\right)^{2}}
$$

can be viewed as an expansion parameter near $k_{c}$ [Eq. (20)]. Expanding $\varepsilon_{ \pm}(k)$ near $k_{c}$ [we should keep only the lowest order in $x$ since the third term of Eq. (27) is already proportional to $d_{a b}$, we find to zeroth order in $x$

$$
\varepsilon_{ \pm}(k)=\frac{\hbar^{2} k^{2}}{2 m}+\frac{1}{2}\left(\epsilon_{b}+\epsilon_{a}\right) \pm\left|d_{a b}\right| \frac{\alpha^{2}-\beta^{2}}{\sqrt{\alpha^{2}+\beta^{2}}} .
$$

The corresponding eigenvectors are 


$$
\left|\psi_{ \pm}\right\rangle=\frac{1}{\sqrt{2}}\left(|-\rangle_{a} \pm|+\rangle_{b}\right),
$$

where $|-\rangle_{a} \rightarrow \varphi_{k, a,-}(x, y)$ and $|+\rangle_{b} \rightarrow \varphi_{k, b,+}(x, y)$ are the eigenstates in Eq. (11). The new eigenstates $\left|\psi_{ \pm}\right\rangle$are zeroth-order linear combinations of the crossing states (remember that the energies are linear in $\alpha d_{a b}$ ). More explicitly,

$$
\left|\psi_{ \pm}\right\rangle=\left[\frac{1}{2}\left(\begin{array}{l}
1 \\
\xi
\end{array}\right) \phi_{a}(y) \pm \frac{1}{2}\left(\begin{array}{c}
1 \\
-\xi
\end{array}\right) \phi_{b}(y)\right] \frac{e^{i k x}}{\sqrt{L_{x}}} .
$$

In a "four-vector notation" we can write

$$
\left|\psi_{ \pm}\right\rangle=\frac{1}{2}\left(\begin{array}{c}
1 \\
\xi \\
\pm 1 \\
\mp \xi
\end{array}\right) \frac{e^{i k x}}{\sqrt{L_{x}}}
$$

As Fig. 2 clearly shows, $\varepsilon_{ \pm}(k)$ [Eq. (29)] approximate well the exact energy dispersions $\varepsilon_{s, s^{\prime}}(k)$ [Eq. (16)] of the problem near $k_{c}$. By using Eq. (29) we can analytically determine the wave vectors $k_{c 1}$ and $k_{c 2}$ relevant for the spin injection problem. This is easily done by imposing $\varepsilon_{F}=\varepsilon_{+}\left(k_{c 1}\right)$ $=\varepsilon_{-}\left(k_{c 2}\right)$ which yields

$$
\frac{\hbar^{2} k_{c 2}^{2}}{2 m}-\frac{\hbar^{2} k_{c 1}^{2}}{2 m}=2\left|d_{a b}\right| \frac{\alpha^{2}-\beta^{2}}{\sqrt{\alpha^{2}+\beta^{2}}} .
$$

For small SO induced interband coupling we look for symmetric solutions around $k_{c}$ [Eq. (20)]: $k_{c 1}=k_{c}-\Delta / 2$ and $k_{c 2}$ $=k_{c}+\Delta / 2$. Equation (33) then gives

$$
\Delta=\frac{2 m\left|d_{a b}\right|}{k_{c} \hbar^{2}} \frac{\alpha^{2}-\beta^{2}}{\sqrt{\alpha^{2}+\beta^{2}}} .
$$

Having determined the wave vectors $k_{c 1}$ and $k_{c 2}$, we can now solve the injection problem. The idea is to expand the incoming electron state, say spin up in channel $a$, in terms of the eigenstates of the SO region. The expansion has to satisfy the boundary conditions (continuity of the wavefunction and flux conservation) at both the entrance and the exit of the SO region.

\section{Boundary conditions}

Here we show that spin injection with energies near the band anticrossing is possible in our system, provided that the SO interband coupling be small compared to the Fermi energy. Details are given in Appendix A.

a. Continuity of the wave function. A spin-up electron in channel $a$ entering the SO region at $x=0$ with an energy $\varepsilon_{F}$ $\sim \varepsilon_{+}\left(k_{c 1}\right)=\varepsilon_{-}\left(k_{c 2}\right)$ has to satisfy

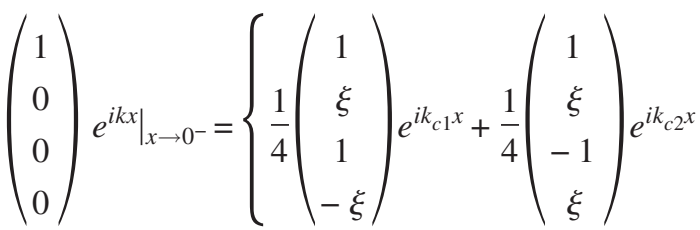

$$
\begin{aligned}
& \left.+\frac{1}{2}\left(\begin{array}{c}
1 \\
-\xi \\
0 \\
0
\end{array}\right) e^{i k_{2} x}\right\}_{x \rightarrow 0^{+}} \text {. }
\end{aligned}
$$

The above condition is clearly fulfilled; a similar condition holds at $x=L$ (see Appendix A).

b. Continuity of the current flow. The continuity of the (non-diagonal) velocity operator ${ }^{55}$ acting on the wave function at $x=0$ which assures current conservation yields

$$
\begin{aligned}
& \left.\left(\begin{array}{c}
\frac{\hbar k_{F}}{m} \\
0 \\
0 \\
0
\end{array}\right) e^{i k x}\right|_{x \rightarrow 0^{-}}=\left\{\begin{array}{c}
\frac{\hbar}{m}\left(k_{c}-\Delta / 2+k_{\mathrm{SO}}\right) \\
\frac{1}{4}\left(k_{c}-\Delta / 2+k_{\mathrm{SO}}\right) \\
\frac{\hbar}{m}\left(k_{c}-\Delta / 2-k_{\mathrm{SO}}\right) \\
-\xi \frac{\hbar}{m}\left(k_{c}-\Delta / 2-k_{\mathrm{SO}}\right)
\end{array}\right) e^{i k_{c 1} x} \\
& +\frac{1}{4}\left(\begin{array}{c}
\frac{\hbar}{m}\left(k_{c}+\Delta / 2+k_{\mathrm{SO}}\right) \\
\frac{\hbar}{m}\left(k_{c}+\Delta / 2+k_{\mathrm{SO}}\right) \\
-\frac{\hbar}{m}\left(k_{c}+\Delta / 2-k_{\mathrm{SO}}\right) \\
\xi \frac{\hbar}{m}\left(k_{c}+\Delta / 2-k_{\mathrm{SO}}\right)
\end{array}\right) e^{i k_{c 2} x} \\
& \left.+\frac{1}{2}\left(\begin{array}{c}
1 \\
-\xi \\
0 \\
0
\end{array}\right) \frac{\hbar}{m}\left(k_{2}-k_{\mathrm{SO}}\right) e^{i k_{2} x}\right\}_{x \rightarrow 0^{+}}
\end{aligned}
$$

which simplifies to 


$$
\left(\begin{array}{c}
\frac{\hbar k_{F}}{m} \\
0 \\
0 \\
0
\end{array}\right)=\frac{1}{2}\left(\begin{array}{c}
\frac{\hbar}{m}\left(k_{c}+k_{2}\right) \\
\xi \frac{\hbar}{m}\left(k_{c}-k_{2}-2 k_{\mathrm{SO}}\right) \\
-\frac{\hbar}{m} \Delta / 2 \\
\xi \frac{\hbar}{m} \Delta / 2
\end{array}\right)=\frac{\hbar k_{F}}{m}\left(\begin{array}{c}
\frac{\left(k_{c}+k_{2}\right)}{2 k_{F}} \\
0 \\
-\frac{\Delta}{4 k_{F}} \\
\xi \frac{\Delta}{4 k_{F}}
\end{array}\right),
$$

where we have used $k_{2}-k_{c}=2 k_{\text {SO }}$ [Eq. (18)]. From Eq. (37) we see that the matching of the derivative is fulfilled provided that $\Delta \ll 4 k_{F}$. As we show later on, this is the case for realistic parameters. The velocity operator matching at $x=L$ holds similarly (see Appendix A).

\section{General spin-rotated state at $x=L$}

After traversing the SO region $a$, say, spin-up electron in channel $a$ is described by the state

$$
\Psi_{\uparrow, a}=\frac{1}{4}\left(\begin{array}{c}
1 \\
\xi \\
1 \\
-\xi
\end{array}\right) e^{i k_{c 1} L}+\frac{1}{4}\left(\begin{array}{c}
1 \\
\xi \\
-1 \\
\xi
\end{array}\right) e^{i k_{c 2} L}+\frac{1}{2}\left(\begin{array}{c}
1 \\
-\xi \\
0 \\
0
\end{array}\right) e^{i k_{2} L}
$$

Straightforward manipulations lead to

$$
\Psi_{\uparrow, a}=\frac{1}{2} e^{i\left(k_{c}+k_{\mathrm{SO}}\right) L}\left(\begin{array}{c}
\cos \left(\theta_{d} / 2\right) e^{-i \theta_{\mathrm{SO}} / 2}+e^{i \theta_{\mathrm{SO}} / 2} \\
\xi\left[\cos \left(\theta_{d} / 2\right) e^{-i \theta_{\mathrm{SO}} / 2}-e^{i \theta_{\mathrm{SO}} / 2}\right] \\
-i \sin \left(\theta_{d} / 2\right) e^{-i \theta_{\mathrm{SO}} / 2} \\
i \xi \sin \left(\theta_{d} / 2\right) e^{-i \theta_{\mathrm{SO}} / 2}
\end{array}\right)
$$

where we have introduced the additional modulation angle

$$
\theta_{d}=\Delta L=\left(\left|d_{a b}\right| / k_{c}\right) \theta_{\mathrm{SO}}\left(\alpha^{2}-\beta^{2}\right) /\left(\alpha^{2}+\beta^{2}\right)
$$

due to SO induced interband mixing. We show in the Appendix A that for $x \geqslant L$, the state

$$
\begin{aligned}
\Psi(x, y)= & \left(\begin{array}{c}
\cos \left(\theta_{d} / 2\right) e^{-i \theta_{\mathrm{SO}} / 2}+e^{i \theta_{\mathrm{SO}} / 2} \\
\xi\left[\cos \left(\theta_{d} / 2\right) e^{-i \theta_{\mathrm{SO}} / 2}-e^{i \theta_{\mathrm{SO}} / 2}\right]
\end{array}\right) \frac{1}{2} e^{i\left(k_{c}+k_{\mathrm{SO}}\right) x} \phi_{a}(y) \\
& +\left(\begin{array}{c}
-i \sin \left(\theta_{d} / 2\right) e^{-i \theta_{\mathrm{SO}} / 2} \\
i \xi \sin \left(\theta_{d} / 2\right) e^{-i \theta_{\mathrm{SO}} / 2}
\end{array}\right) \frac{1}{2} e^{i\left(k_{c}-k_{\mathrm{SO}}\right) x} \phi_{b}(y)
\end{aligned}
$$

satisfies the proper boundary condition for the velocity operator [note that setting $x=L$ in Eq. (41) gives Eq. (39), thus fulfilling the continuity of the wave function at this interface]. Equation (41) shows that upon traversing the SO active region of length $L$, a spin-up electron in the incoming channel $a$, acquires a spin-down component in the same channel and, more importantly, coherently transfers into channel $b$. This coherent transfer from channel $a$ to channel $b$ is solely due to the SO induced interband coupling near $k_{c}$, described by the mixing angle $\theta_{d}$. Hence, a weak SO-induced interchannel mixing-rather than being detrimental to transport-offers a unique possibility for further spin modulating the electron flow.

\section{SO transfer matrix: Coupled channels}

Similarly to the uncoupled-channel case, here we can also define a SO transfer matrix $U_{\mathrm{SO}}^{c}$ describing the effect of the SO interaction on electrons impinging near the band crossing at $k_{c}$. This transfer matrix is readily contructed in terms of the column vectors similar to the one in Eq. (39), which describes how a spin up electron in channel $a$ evolves upon crossing the $\mathrm{SO}$ region. We obtain

$$
\mathbf{U}_{\mathrm{SO}}^{c c}=\frac{1}{2} e^{i k_{c} L}\left(\begin{array}{cccc}
\cos \left(\theta_{d} / 2\right)+e^{i \theta_{\mathrm{SO}}} & \xi^{*}\left[\cos \left(\theta_{d} / 2\right)-e^{i \theta_{\mathrm{SO}}}\right] & -i \sin \left(\theta_{d} / 2\right) & i \xi^{*} \sin \left(\theta_{d} / 2\right) \\
\xi\left[\cos \left(\theta_{d} / 2\right)-e^{\left.i \theta_{\mathrm{SO}}\right]}\right. & \cos \left(\theta_{d} / 2\right)+e^{i \theta_{\mathrm{SO}}} & -i \xi \sin \left(\theta_{d} / 2\right) & i \sin \left(\theta_{d} / 2\right) \\
-i \sin \left(\theta_{d} / 2\right) & -i \xi^{*} \sin \left(\theta_{d} / 2\right) & \cos \left(\theta_{d} / 2\right)+e^{-i \theta_{\mathrm{SO}}} & -\xi^{*}\left[\cos \left(\theta_{d} / 2\right)-e^{\left.-i \theta_{\mathrm{SO}}\right]}\right. \\
i \xi \sin \left(\theta_{d} / 2\right) & i \sin \left(\theta_{d} / 2\right) & -\xi\left[\cos \left(\theta_{d} / 2\right)-e^{\left.-i k \theta_{\mathrm{SO}}\right]}\right. & \cos \left(\theta_{d} / 2\right)+e^{-i \theta_{\mathrm{SO}}}
\end{array}\right),
$$

where the modulation angles $\theta_{\mathrm{SO}}$ and $\theta_{d}$ are given in Eqs. (22) and (40), respectively. We should keep in mind that Eq. (42) describes electrons traversing the SO region with energies near the crossing energy. As we discuss later on, the SO transfer matrix above is also useful for spin-rotating entangled and/or unentangled electron pairs injected into a four-terminal geometry (beam splitter). The idea is that $U^{c c}$ operates on the member of the pair traversing the SO region.
Note that the transfer matrix in Eq. (42) reduces to that of the uncoupled case, Eq. (24), for $\alpha=\beta\left(\theta_{d}=0\right)$. Next we estimate the magnitude of the spin rotations we have described here.

\section{E. Single spin rotation $\theta_{\text {SO }}$ for coupled channels $\left(d_{a b} \neq 0\right)$ with $\boldsymbol{\alpha}=\boldsymbol{\beta}$}

Here the calculation is simpler since the bands do not anticross even for nonzero $d_{a b}$ as we discussed earlier. The 
crossing wave vector $\bar{k}_{c}$ for $\alpha=\beta$ is determined from Eq. (17). For instance, the $k>0$ crossing is obtained by setting $\varepsilon_{+,-}\left(\bar{k}_{c}\right)=\varepsilon_{-,+}\left(\bar{k}_{c}\right)$ which gives

$$
\bar{k}_{c}=\frac{1}{2 \sqrt{2} \alpha} \sqrt{\left(\epsilon_{b}-\epsilon_{a}\right)^{2}+8 \alpha^{2}\left|d_{a b}\right|^{2}} .
$$

For $d_{a b}=0$ and $\alpha=\beta$ the above wave vector reduces to $k_{c}$ defined in Eq. (20).

By expanding the incoming electron states into the exact eigenstates derived in Sec. II A we can obtain the modulation angle $\theta_{\mathrm{SO}}=2 \sqrt{2} \mathrm{~mL} / \hbar^{2}$. Note that $\theta_{d}=0$ for $\alpha=\beta$. Interestingly, the matching of the boundary conditions here and the general state at $x=L$ can be straightforwardly obtained from the $\alpha \neq \beta$ case by setting $\Delta=0$ (or equivalently, $\theta_{d}=0$ ). However, it is important to note that the crossing wave vector is now $\bar{k}_{c}$ (not $k_{c}$ ) and that $k_{2}-\bar{k}_{c}=2 k_{\mathrm{SO}}$, where $k_{\mathrm{SO}}$ is calculated for $\alpha=\beta$. Note also that only one modulation angle $\theta_{\mathrm{SO}}$ is present for the tuned-coupling case $\alpha=\beta$. Hence, this case is similar to the uncoupled channel problem treated by Datta and Das, ${ }^{29}$ even though here $d_{a b} \neq 0$. The identical coupling strengths makes the problem similar to that of the uncoupled channels; however, the rotation angle is now renormalized.

\section{F. Estimates for the modulating angles $\boldsymbol{\theta}_{\mathrm{SO}}$ and $\boldsymbol{\theta}_{\boldsymbol{d}}$}

Simple estimates for the spin-rotation angle $\theta_{\text {SO }}$ and the mixing angle $\theta_{d}$ can be obtained by assuming a hard-wall transverse confinement of width $w$. Using the well-known analytical results for the wire problem, we find $d_{a b}=8 / 3 w$ for the interband mixing and $\epsilon_{b}=3 \pi^{2} \hbar^{2} / 2 m w^{2}$ (assuming $\left.\epsilon_{a} \equiv 0\right)$. The quantity $\epsilon_{\mathrm{SO}} \equiv \hbar^{2} k_{\mathrm{SO}}^{2} / 2 m=m\left(\alpha^{2}+\beta^{2}\right) / 2 \hbar^{2}$ sets an energy scale in our problem. For the sake of concreteness, let us choose $\epsilon_{b}=16 \epsilon_{\mathrm{SO}}$ which leads to $\sqrt{\alpha^{2}+\beta^{2}}$ $=(\sqrt{3 / 2} \pi / 4) \hbar^{2} / m w=2.44 \times 10^{-2} \mathrm{eV} \mathrm{nm} \quad$ (and $\epsilon_{\mathrm{SO}}$ $\sim 0.2 \mathrm{meV}$ ) for $m=0.05 m_{0}$ (see Ref. 48) and $w=60 \mathrm{~nm}$. The energy at the band crossing points is then $\varepsilon_{a}^{-}\left(k_{c}\right)=24 \epsilon_{\mathrm{SO}}$ $\sim 4.8 \mathrm{meV}$; note that for Fermi energies close to this value, $\mathrm{SO}$ induced channel-mixing effects are important. From Eq. (20) we find $k_{c}=8 \epsilon_{\mathrm{SO}} / \sqrt{\alpha^{2}+\beta^{2}}$. Assuming an active SO region of length $L=69 \mathrm{~nm}$ we can estimate the spin-rotation angles; we find $\theta_{\mathrm{SO}}=\pi$. To obtain $\theta_{d}=\left(d_{a b} / k_{c}\right) \theta_{\mathrm{SO}}\left(\alpha^{2}\right.$ $\left.-\beta^{2}\right) /\left(\alpha^{2}+\beta^{2}\right)$ we need an estimate for $\beta$. To estimate the Dresselhaus coefficient in a quantum well geometry we use $\beta=\tilde{\gamma}\left\langle k_{z}^{2}\right\rangle$, where $\left\langle k_{z}^{2}\right\rangle$ denotes the expectation value of the wave vector component along the growth direction. For the lowest infinite-well eigenstate we find $\left\langle k_{z}^{2}\right\rangle=(\pi / w)^{2}$. The coefficient $\tilde{\gamma}$ is typically $\approx 25 \mathrm{eV}^{3}$ (Refs. 56-58) which yields $\beta \approx 10^{-5} \mathrm{eV} \mathrm{nm}$. Hence, for such III-V materials we can neglect $\beta$ and use $\theta_{d}=\left(d_{a b} / k_{c}\right) \theta_{\mathrm{SO}}$, which gives $\theta_{d}$ $=\pi / 2$ since $d_{a b} / k_{c}=2 /\left(3 k_{\mathrm{SO}} w\right) \sim 0.5$.

In order to obtain comparable Rashba and Dresselhaus coupling strengths, we could use a setup with wider wires and materials with a larger effective mass. ${ }^{49}$ In addition, we could consider an inhomogeneous beam splitter with a different material with larger Dresselhaus coupling in one of the incoming arms. Note that the possibility of tuned couplings $\alpha=\beta$ is very attractive since in this case the spin of the electron propagating in the SO coupled channels is insensitive to nonmagnetic impurity scattering (Sec. II A), i.e., the spinor is $k$ independent for $\alpha=\beta$.

We stress that the modulation angles $\theta_{\text {So }}$ and $\theta_{d}$ can, in principle, be tuned independently via a proper gating structure. This could involve, for instance, both side (top) and back gates to induce changes in the channel width $w$ (confining potential) and the Rashba constant. The above conservative estimates suggests that the spin rotations we are considering here are sizable. Finally, we note that for the above parameters $\Delta / 4 k_{F} \sim 0.05 \ll 1$, which justifies the approximation made in the velocity operator matching [Eq. (37)].

\section{TRANSPORT PROPERTIES: CURRENT AND NOISE}

In what follows we calculate the current and its dynamic fluctuations (shot noise) for electrons traversing a beam splitter. We use the scattering approach of Landauer and Büttiker. ${ }^{59}$ We consider injection of (i) electron pairs (singlet and triplets) from an "entangler" tunnel-coupled to the incoming leads of the beam splitter and (ii) spin-polarized electrons from Fermi-liquid leads which are assumed to be thermal reservoirs each held at a given chemical potential. For a calculation of shot noise for entangled electrons in a beam-splitter where a Berry phase provides an additional modulation, see Ref. 60.

\section{A. Scattering approach: basics}

Here we briefly outline the scattering-matrix formulation for current and noise. ${ }^{59}$

\section{Current}

Within the Landauer-Büttiker approach, the transport properties of a mesoscopic system are expressed in terms of the scattering matrix $\mathbf{s}_{\gamma \mu}$ connecting the many incoming and outgoing attached leads. The current operator in lead $\gamma$ is

$$
\hat{I}_{\gamma}(t)=\frac{e}{h \nu} \sum_{\alpha \beta \sigma \sigma^{\prime}} \sum_{\varepsilon \varepsilon^{\prime}} A_{\alpha, \beta}^{\sigma, \sigma^{\prime}}\left(\gamma ; \varepsilon, \varepsilon^{\prime}\right) e^{i\left(\varepsilon-\varepsilon^{\prime}\right) t / \hbar} a_{\alpha \sigma}^{\dagger}(\varepsilon) a_{\beta \sigma^{\prime}}\left(\varepsilon^{\prime}\right),
$$

with

$$
A_{\alpha, \beta}^{\sigma, \sigma^{\prime}}\left(\gamma ; \varepsilon, \varepsilon^{\prime}\right)=\delta_{\sigma, \sigma^{\prime}} \delta_{\gamma, \alpha} \delta_{\gamma, \beta}-\sum_{\sigma^{\prime \prime}} s_{\gamma \alpha ; \sigma \sigma^{\prime \prime}}^{*}(\varepsilon) s_{\gamma \beta ; \sigma^{\prime \prime} \sigma^{\prime}}\left(\varepsilon^{\prime}\right),
$$

where $\sigma=\uparrow, \downarrow$ is the relevant spin component along a proper quantization direction (" $x, y$, or $z$ "). We have introduced the creation (annihilation) fermionic operator $a_{\alpha \sigma}^{\dagger}(\varepsilon)\left[a_{\alpha \sigma}(\varepsilon)\right]$ for an electron with energy $\varepsilon$ in lead $\alpha$, which satisfy the anticommutation relation $\left\{a_{\alpha \sigma}^{\dagger}(\varepsilon), a_{\alpha^{\prime} \sigma^{\prime}}\left(\varepsilon^{\prime}\right)\right\}=\delta_{\alpha \alpha^{\prime}} \delta_{\sigma \sigma^{\prime}} \delta_{\varepsilon \varepsilon^{\prime}}$ We have considered beam splitter leads with discrete longitudinal energy levels $\varepsilon, \varepsilon^{\prime}$. This yields the factor $\nu$ $=\left(L_{x} / 2 \pi \hbar\right) \sqrt{m / 2 E_{F}}$ in Eq. (44), which actually is the 1D density of states for only forward propagating states (positive momenta). In the standard expression for the current with continuous energies, ${ }^{59}$ this factor cancels with the density of 
states appearing when transforming discrete states into continuous ones. For a study of noise in a beam splitter with continuous energies, see Refs. 61 and 62. We discuss in more details the transition from the discrete case to the continuous one in Sec. III E.

\section{Shot noise}

At a time $t$, the current fluctuation about its average in lead $\gamma$ is $\delta \hat{I}_{\gamma}(t)=\hat{I}_{\gamma}(t)-\left\langle\hat{I}_{\gamma}\right\rangle$. In a multiple-lead configuration, the shot noise between leads $\gamma$ and $\mu$ is defined as the Fourier transform of the symmetrized current-current autocorrelation function

$$
S_{\gamma \mu}(\omega)=\frac{1}{2} \int\left\langle\delta \hat{I}_{\gamma}(t) \delta \hat{I}_{\mu}\left(t^{\prime}\right)+\delta \hat{I}_{\mu}\left(t^{\prime}\right) \delta \hat{I}_{\gamma}(t)\right\rangle e^{i \omega t} d t .
$$

The angle brackets in Eq. (46) stand for either (i) a quantum mechanical expectation value between two-particle states or (ii) a standard ensemble average (thermal reservoirs). Note that the nonequilibrium current noise defined above arises physically from the discrete nature of the charge flow in the system. This is strictly true only at zero temperatures; at finite temperatures Eq. (46) contains also thermal noise.

\section{Beam-splitter scattering matrix}

To calculate the noise from Eq. (46) we need to specify the beam splitter scattering matrix. For a symmetric beam splitter without SO interaction and single-mode channels, we have the scattering matrix ${ }^{3}$

$$
\mathbf{s}=\left(\begin{array}{cccc}
0 & 0 & s_{13} & s_{14} \\
0 & 0 & s_{23} & s_{24} \\
s_{31} & s_{32} & 0 & 0 \\
s_{41} & s_{42} & 0 & 0
\end{array}\right)=\left(\begin{array}{cccc}
0 & 0 & r & t \\
0 & 0 & t & r \\
r & t & 0 & 0 \\
t & r & 0 & 0
\end{array}\right),
$$

that is, the beam splitter transmits electrons between leads 1 and 3 and leads 2 and 4 with amplitude $r$ and between leads 2 and 3 and leads 2 and 4 with amplitude $t$. Note that backscattering is neglected in s; see Sec. III C and Appendix C 1 for a beamsplitter including backscattering effects. If the incoming or outgoing leads have more than one mode (i.e., many quantized channels) we can, in a first approximation, assume that the beamsplitter does not mix the orbital channels so that Eq. (47) holds true for each of the modes separately.

Interestingly, in the presence of SO interaction in lead 1, Fig. 1, we can define an extended beam-splitter scattering matrix to incorporate the spin rotation described by the SO transfer matrix $\mathbf{U}_{\text {So. }}$. Since an electron in lead 1 undergoes a spin rotation described by $\mathbf{U}_{\mathrm{SO}}$, we can redefine the matrix elements $s_{13}=s_{31}$ and $s_{14}=s_{41}$ as $4 \times 4$ matrices

$$
\mathbf{s}_{13}^{\mathrm{SO}}=\mathbf{s}_{31}^{\mathrm{SO}}=s_{13} \mathbf{U}_{\mathrm{SO}},
$$

and

$$
\mathbf{s}_{14}^{\mathrm{SO}}=\mathbf{s}_{41}^{\mathrm{SO}}=s_{14} \mathbf{U}_{\mathrm{SO}},
$$

to incorporate the effects of the SO interaction. Note that $\mathbf{U}_{\text {SO }}$ is given by Eqs. (24) and (42) for the uncoupled and the coupled two-channel cases, respectively. The other elements in $\mathbf{s}$ remain unaltered except that they are now $4 \times 4$ matrices, e.g., $\mathbf{s}_{24}=\mathbf{s}_{42}=s_{14} \mathbf{1}$, where $\mathbf{1}$ denotes the $4 \times 4$ unit matrix. Note that the new beam splitter scattering matrix $\mathbf{s}^{\mathrm{SO}}$ incorporating the SO effects in lead 1 as defined above is a $16 \times 16$ object as opposed to the $4 \times 4$ matrix in Eq. (47).

\section{B. Shot noise for electron pairs: Singlet and triplet states}

We assume that an entangler ${ }^{5-25}$ is placed just before leads 1 and 2, Fig. 1(a). ${ }^{63}$ Below we calculate the noise for the states

$$
\left.\begin{array}{c}
|S\rangle \\
\left|T_{e_{i}}\right\rangle
\end{array}\right\}=\frac{1}{\sqrt{2}}\left[a_{1 \uparrow}^{\dagger}\left(\varepsilon_{1}\right) a_{2 \downarrow}^{\dagger}\left(\varepsilon_{2}\right) \mp a_{1 \downarrow}^{\dagger}\left(\varepsilon_{1}\right) a_{2 \uparrow}^{\dagger}\left(\varepsilon_{2}\right)\right]|0\rangle,
$$

$$
\left|T_{u_{\sigma, i}}\right\rangle=a_{1 \sigma}^{\dagger}\left(\varepsilon_{1}\right) a_{2 \sigma}^{\dagger}\left(\varepsilon_{2}\right)|0\rangle, \quad \sigma=\uparrow, \downarrow,
$$

where $|0\rangle$ denotes the ground state (filled) Fermi sea of the leads and $i=x, y, z$ any particular quantization axis. The states $|S\rangle$ and $\left|T_{e_{i}}\right\rangle$ are entangled singlet and triplet, respectively, while $\left|T_{u_{\uparrow, i}}\right\rangle$ and $\left|T_{u_{\downarrow, i}}\right\rangle$ are unentangled triplets. Here we consider zero temperatures, zero applied voltages, and zero frequencies. In this limit the Fermi sea is completely inert (noiseless) and the noise in the system is solely due to the injected pairs above the Fermi surface. ${ }^{6}$ To determine the shot noise we essentially evaluate matrix elements of the general form

$$
\begin{aligned}
& \langle 0| a_{\mu, \sigma_{\mu}}\left(\varepsilon_{\mu}\right) a_{\nu, \sigma_{\nu}}\left(\varepsilon_{\nu}\right) a_{\alpha, \sigma^{\prime}}^{\dagger}(\varepsilon) a_{\beta, \sigma^{\prime}}\left(\varepsilon^{\prime}\right) \\
& \times a_{\alpha^{\prime}, \sigma^{\prime \prime}}^{\dagger}\left(\varepsilon^{\prime \prime}\right) a_{\beta^{\prime}, \sigma^{\prime \prime \prime}}\left(\varepsilon^{\prime \prime \prime}\right) a_{\gamma, \sigma_{\gamma}}^{\dagger}\left(\varepsilon_{\gamma}\right) a_{\eta, \sigma_{\eta}}^{\dagger}\left(\varepsilon_{\eta}\right)|0\rangle,
\end{aligned}
$$

appearing in the noise definition (46). This is most systematically done via Wick's theorem since the object in Eq. (48) resembles a four-particle Green function (see Appendix D for details).

\section{General noise formulas: Single-channel case}

For the injected singlet and the triplets in Eq. (50) we find the following expressions for the zero-frequency noise between leads $\gamma$ and $\mu$ : 


$$
\begin{aligned}
S_{\gamma \mu}^{S / T_{e_{i}}=} & \frac{e^{2}}{2 h \nu}\left[\sum_{\alpha=1,2, \beta=1 \ldots 4, \sigma^{\prime}, \sigma} A_{\alpha, \beta}^{\sigma, \sigma^{\prime}}\left(\gamma ; \varepsilon_{\alpha}, \varepsilon_{\alpha}\right) A_{\beta, \alpha}^{\sigma^{\prime}, \sigma}\left(\mu ; \varepsilon_{\alpha}, \varepsilon_{\alpha}\right)+\sum_{\alpha \neq \beta=1,2, \sigma}\left[ \pm A_{\alpha, \beta}^{\sigma, \sigma}\left(\gamma ; \varepsilon_{\alpha}, \varepsilon_{\alpha}\right) A_{\beta, \alpha}^{-\sigma,-\sigma}\left(\mu ; \varepsilon_{\beta}, \varepsilon_{\beta}\right)\right.\right. \\
& \left.-A_{\alpha, \beta}^{\sigma,-\sigma}\left(\gamma ; \varepsilon_{\alpha}, \varepsilon_{\alpha}\right) A_{\beta, \alpha}^{-\sigma, \sigma}\left(\mu ; \varepsilon_{\beta}, \varepsilon_{\beta}\right)\right] \delta_{\varepsilon_{\alpha}, \varepsilon_{\beta}} \mp \sum_{\alpha \neq \beta=1,2 ; \sigma} A_{\alpha, \alpha}^{\sigma,-\sigma}\left(\gamma ; \varepsilon_{\alpha}, \varepsilon_{\alpha}\right) A_{\beta, \beta}^{-\sigma, \sigma}\left(\mu ; \varepsilon_{\beta}\right) \\
& +\frac{1}{2} \sum_{\alpha \neq \beta=1,2 ; \sigma} A_{\alpha, \alpha}^{\sigma, \sigma}\left(\gamma ; \varepsilon_{\alpha}, \varepsilon_{\alpha}\right) A_{\beta, \beta}^{-\sigma,-\sigma}\left(\mu ; \varepsilon_{\beta}, \varepsilon_{\beta}\right)-\frac{1}{2} \sum_{\alpha, \beta=1,2 ; \sigma} A_{\alpha, \alpha}^{\sigma, \sigma}\left(\gamma ; \varepsilon_{\alpha}, \varepsilon_{\alpha}\right) A_{\beta, \beta}^{\sigma, \sigma}\left(\mu ; \varepsilon_{\beta}\right) \\
& \left.-\frac{1}{2} \sum_{\alpha=1,2 ; \sigma} A_{\alpha, \alpha}^{\sigma, \sigma}\left(\gamma ; \varepsilon_{\alpha}, \varepsilon_{\alpha}\right) A_{\alpha, \alpha}^{-\sigma,-\sigma}\left(\mu ; \varepsilon_{\alpha}, \varepsilon_{\alpha}\right)\right]
\end{aligned}
$$

and

$$
\begin{aligned}
S_{\gamma \mu}^{T_{u_{\sigma, i}}}= & \frac{e^{2}}{h \nu}\left[\sum_{\alpha=1,2, \beta=1 \ldots 4, \sigma^{\prime}} A_{\alpha, \beta}^{\sigma, \sigma^{\prime}}\left(\gamma ; \varepsilon_{\alpha}, \varepsilon_{\alpha}\right) A_{\beta, \alpha}^{\sigma^{\prime}, \sigma}\left(\mu ; \varepsilon_{\alpha}, \varepsilon_{\alpha}\right)\right. \\
& \left.-\sum_{\alpha, \beta=1,2} A_{\alpha, \beta}^{\sigma, \sigma}\left(\gamma ; \varepsilon_{\alpha}, \varepsilon_{\alpha}\right) A_{\beta, \alpha}^{\sigma, \sigma}\left(\mu ; \varepsilon_{\beta}, \varepsilon_{\beta}\right) \delta_{\varepsilon_{\alpha}, \varepsilon_{\beta}}\right] .
\end{aligned}
$$

Note that Eqs. (52) and (53) do not depend on the particular form (47) of the beam-splitter scattering matrix and the quantization axis chosen. In what follows we present explicit formulas for the noise derived from Eqs. (52) and (53). We also determine the noise for entangled and unentangled states defined along distinct quantization axes $(i=x, y$, and $z)$ and for the Bell states. Later on we present similar results for the two-channel case as well.

\section{Specific formulas: Uncoupled-channel case}

In the absence of $\mathrm{SO}$ induced interband coupling (uncoupled-channel case), the channels $a$ and $b$ are independent within lead 1 . That is, if electrons are injected only in the channel $a$ of lead 1, they will remain in that channel while propagating through the length $L$ of the $\mathrm{SO}$ region in that lead. If fact, the channel index remains unaltered as the electrons traverse the beam splitter since we assume the the beamsplitter does not mix the channels. We present below results for electron pairs injected only in channel $a$ of the incoming leads. The case with two pairs injected into channels $a$ and $b$ is straighforward (factor of 2) since no SO interband mixing is considered here. However, as we discuss later on, in the coupled-channel case injection into just one of the channels is significantly different from injection into both channels (not just a factor of 2 as here). Below we detail the calculation of the noise from Eqs. (52) and (53). To cal-

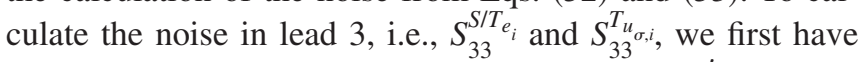
to determine the relevant elements $A_{\alpha, \beta}^{\sigma, \sigma^{\prime}}\left(3 ; \varepsilon_{\alpha}, \varepsilon_{\alpha}\right)$ [Eq. (45)] appearing in these quantities. Since our scattering matrix is assumed to be independent of the energy, so is $A_{\alpha, \beta}^{\sigma, \sigma^{\prime}}\left(3 ; \varepsilon_{\alpha}, \varepsilon_{\alpha}\right)=A_{\alpha, \beta}^{\sigma, \sigma^{\prime}}(3)$.

a. Quantization axis along $z$. For the specific forms

$$
\mathbf{s}_{13}^{\mathrm{SO}}=\left(\begin{array}{cc}
r \cos \left(\theta_{\mathrm{SO}} / 2\right) & r \sin \left(\theta_{\mathrm{SO}} / 2\right) \\
-r \sin \left(\theta_{\mathrm{SO}} / 2\right) & r \cos \left(\theta_{\mathrm{SO}} / 2\right)
\end{array}\right)
$$

and

$$
\mathbf{s}_{14}^{\mathrm{SO}}=\left(\begin{array}{cc}
t \cos \left(\theta_{\mathrm{SO}} / 2\right) & t \sin \left(\theta_{\mathrm{SO}} / 2\right) \\
-t \sin \left(\theta_{\mathrm{SO}} / 2\right) & t \cos \left(\theta_{\mathrm{SO}} / 2\right)
\end{array}\right),
$$

the only nonzero $A_{\alpha, \beta}^{\sigma, \sigma^{\prime}}(3)$ 's are

$$
\begin{aligned}
A_{1,2}^{\uparrow, \uparrow}(3) & =-s_{31 ; \downarrow \uparrow}^{*} s_{32 ; \downarrow \uparrow}-s_{31 ; \uparrow \uparrow}^{*} s_{32 ; \uparrow \uparrow}=-r^{*} t \cos \left(\theta_{\mathrm{SO}} / 2\right) \\
& =A_{1,2}^{\downarrow, \downarrow}(3), \\
A_{1,2}^{\uparrow, \downarrow}(3) & =-s_{31 ; \downarrow \uparrow}^{*} s_{32 ; \downarrow \downarrow}-s_{31 ; \uparrow \uparrow}^{*} s_{32 ; \uparrow \downarrow}=r^{*} t \sin \left(\theta_{\mathrm{SO}} / 2\right) \\
& =-A_{1,2}^{\uparrow, \downarrow}(3), \\
A_{2,2}^{\uparrow, \uparrow}(3) & =-s_{32 ; \downarrow \uparrow}^{*} s_{32 ; \downarrow \uparrow}-s_{32 ; \uparrow \uparrow}^{*} s_{32 ; \uparrow \uparrow}=-|t|^{2}=A_{2,2}^{\downarrow, \downarrow}(3) .
\end{aligned}
$$

Note that in the above we have chosen the $\sigma$ index in $\mathbf{s}^{\text {SO }}$ to be that of the $z$ component of the spin $\sigma \rightarrow \sigma_{z}=\uparrow, \downarrow$, i.e., we have set the quantization axis to be $z$, Fig. 1. Hence, the entangled and non-entangled triplet states here refer to this basis: $S_{33}^{T_{e_{z}}}$ and $S_{33}^{T_{u_{\sigma, z}}}$; the noise for the singlet state $S_{33}^{S}$ is the same for all quantization axes. Plugging in the above $A_{\alpha, \beta}^{\sigma, \sigma^{\prime}}(3)$ 's into Eq. (52) we find

$$
\begin{aligned}
S_{33}^{S / T} e_{z}\left(\theta_{\mathrm{SO}}\right)= & \frac{2 e^{2} R T}{h \nu}\left[1 \pm \cos \left(\theta_{\mathrm{SO}} / 2\right) \cos \left(\theta_{\mathrm{SO}} / 2\right) \delta_{\varepsilon_{1}, \varepsilon_{2}}\right. \\
& \left.-\sin \left(\theta_{\mathrm{SO}} / 2\right) \sin \left(\theta_{\mathrm{SO}} / 2\right) \delta_{\varepsilon_{1}, \varepsilon_{2}}\right],
\end{aligned}
$$

where we have defined the transmission and reflection probabilities $T=|t|^{2}$ and $R=|r|^{2}$, respectively. Since $R+T=1$, further simplifications lead to

$$
\begin{gathered}
S_{33}^{S}\left(\theta_{\mathrm{SO}}\right)=\frac{2 e^{2}}{h \nu} T(1-T)\left[1+\cos \left(\theta_{\mathrm{SO}}\right) \delta_{\varepsilon_{1}, \varepsilon_{2}}\right], \\
S_{33}^{T_{e}}{ }_{z}\left(\theta_{\mathrm{SO}}\right)=\frac{2 e^{2}}{h \nu} T(1-T)\left(1-\delta_{\varepsilon_{1}, \varepsilon_{2}}\right) .
\end{gathered}
$$

Similarly, we find 
$S_{33}^{T_{u_{1, z}}}\left(\theta_{\mathrm{SO}}\right)=S_{33^{\prime, z}}^{T_{u_{1}}}\left(\theta_{\mathrm{SO}}\right)=\frac{2 e^{2}}{h \nu} T(1-T)\left[1-\cos ^{2}\left(\theta_{\mathrm{SO}} / 2\right) \delta_{\varepsilon_{1}, \varepsilon_{2}}\right]$,

for the unentangled triplets with spin polarization along $z$.

The above formulas have been derived for the case where the injected electrons in arm 1 and 2 have the infinitely sharp energies $\varepsilon_{1}$ and $\varepsilon_{2}$. In Sec. III $\mathrm{E}$ we consider the case where the injected electrons are described by Lorentzian wave packets of width $\gamma$ centered on $\varepsilon_{1}$ and $\varepsilon_{2}$. We then find, in the continuous limit, that $\delta_{\varepsilon_{1}, \varepsilon_{2}}$ in Eqs. (60)-(62) is replaced by the function $H(\Delta)=\gamma^{2} /\left(\Delta^{2} / 4+\gamma^{2}\right)$ where $\Delta=\varepsilon_{1}-\varepsilon_{2}$. This function, which interpolates between $H=1$ when $\Delta \ll \gamma$ and $H=0$ when $\Delta \gg \gamma$, corresponds to the one appearing in Eq. (28) of Ref. 61.

b. Quantization axis along $y$. To obtain the corresponding noise expressions for injected electron pairs with spin polarization defined along the $y$ we have to first rewrite $\mathbf{s}^{\mathrm{SO}}$ in the basis of $\sigma_{y}:|\uparrow\rangle_{y}=\left(|\uparrow\rangle_{z}+i|\downarrow\rangle_{z}\right) / \sqrt{2},|\downarrow\rangle_{y}=\left(|\uparrow\rangle_{z}-i|\downarrow\rangle_{z}\right) / \sqrt{2}$. For instance, $\mathbf{s}_{13}^{\mathrm{SO}}$ becomes

$$
\mathbf{s}_{13, y}^{\mathrm{SO}}=\left(\begin{array}{cc}
r \exp \left(i \theta_{\mathrm{SO}} / 2\right) & 0 \\
0 & r \exp \left(-i \theta_{\mathrm{SO}} / 2\right)
\end{array}\right) .
$$

Now the only nonzero elements are $A_{1,2}^{\uparrow \uparrow}(3)$ $=-r^{*} t \exp \left(-i \theta_{\mathrm{SO}} / 2\right)=\left[A_{2,1}^{\downarrow, \downarrow}(3)\right]^{*} \quad$ and $\quad A_{2,1}^{,, \uparrow}(3)$ $=-r t^{*} \exp \left(-i \theta_{\mathrm{SO}} / 2\right)=\left[A_{1,2}^{,,}(3)\right]^{*}$. Substituting these terms into the general Eqs. (52) and (53), we find

$$
S_{33}^{S / T} e_{y}\left(\theta_{\mathrm{SO}}\right)=\frac{2 e^{2}}{h \nu} T(1-T)\left[1 \pm \cos \left(\theta_{\mathrm{SO}}\right) \delta_{\varepsilon_{1}, \varepsilon_{2}}\right]
$$

and

$$
S_{33}^{T_{u \uparrow}, y}\left(\theta_{\mathrm{SO}}\right)=S_{33}^{T_{u_{\downarrow}}}\left(\theta_{\mathrm{SO}}\right)=\frac{2 e^{2}}{h \nu} T(1-T)\left(1-\delta_{\varepsilon_{1}, \varepsilon_{2}}\right) .
$$

c. Bell states. For completeness we also calculate the noise for injected Bell pairs ${ }^{64}$ (maximally entangled states) described by

$$
\begin{gathered}
\left|\Psi_{0}\right\rangle=|S\rangle, \\
\left|\Psi_{1}\right\rangle=\left|T_{e_{i}}\right\rangle, \\
\left|\Psi_{2}\right\rangle=\frac{1}{\sqrt{2}}\left(\left|T_{u \uparrow, i}\right\rangle+\left|T_{u \downarrow, i}\right\rangle\right), \\
\left|\Psi_{3}\right\rangle=\frac{1}{\sqrt{2}}\left(\left|T_{u \uparrow, i}\right\rangle-\left|T_{u \downarrow, i}\right\rangle\right) .
\end{gathered}
$$

The Bell states above are defined with respect to an arbitrary quantization axis. The noise expressions for the first two Bell states are the same as those of the singlet and the entangled triplet derived above. The noise for the states $\left|\Psi_{2}\right\rangle$ and $\left|\Psi_{3}\right\rangle$ can be easily determined in terms of the results for the unentangled triplet states for particular quantization axes. Let us consider the quantization axis along $z$, for concreteness.
The procedure is straightforward.

(i) After traversing the $\mathrm{SO}$ region, the injected unentangled triplet state $\left|T_{u_{\uparrow, z}}\right\rangle$ becomes $\left|T_{u_{\uparrow, z}}\right\rangle^{L}=\mathbf{U}_{\mathrm{SO}}^{a}\left|T_{u_{\uparrow, z}}\right\rangle$ and can be decomposed as

$$
\left|T_{u_{\uparrow, z}}\right\rangle^{L}=\left[\cos \left(\theta_{\mathrm{SO}} / 2\right)|\uparrow\rangle_{1 a, z}-\sin \left(\theta_{\mathrm{SO}} / 2\right)|\downarrow\rangle_{1 a, z}\right] \otimes|\uparrow\rangle_{2 a, z}
$$

or

$$
\left|T_{u_{\uparrow, z}}\right\rangle^{L}=\cos \left(\theta_{\mathrm{SO}} / 2\right)|\uparrow \uparrow\rangle_{a a, z}-\sin \left(\theta_{\mathrm{SO}} / 2\right)|\downarrow \uparrow\rangle_{a a, z},
$$

where we have used the shorthand notation $|\uparrow \uparrow\rangle_{a a, z}$ $=|\uparrow\rangle_{1 a, z} \otimes|\uparrow\rangle_{2 a, z}=\left|T_{u_{\uparrow, z}}\right\rangle$, to denote the tensor product state with one electron injected into the channel $a$ of lead 1 and another in the channel $a$ of lead 2. Similarly,

$$
\left|T_{u_{\downarrow z}}\right\rangle^{L}=\sin \left(\theta_{\mathrm{SO}} / 2\right)|\uparrow \downarrow\rangle_{a a, z}+\cos \left(\theta_{\mathrm{SO}} / 2\right)|\downarrow \downarrow\rangle_{a a, z} .
$$

(ii) Now, we rewrite the rotated Bell state $\left|\Psi_{2}\right\rangle^{L}$ explicitly

$$
\begin{aligned}
\left|\Psi_{2}\right\rangle^{L}= & \cos \left(\theta_{\mathrm{SO}} / 2\right) \frac{1}{\sqrt{2}}\left(|\uparrow \uparrow\rangle_{a a, z}+|\downarrow \downarrow\rangle_{a a, z}\right) \\
& +\sin \left(\theta_{\mathrm{SO}} / 2\right) \frac{1}{\sqrt{2}}\left(|\uparrow \downarrow\rangle_{a a, z}-|\downarrow \uparrow\rangle_{a a, z}\right) .
\end{aligned}
$$

(iii) The noise in lead 3 corresponding to $\left|\Psi_{2}\right\rangle^{L}$ is determined from the expectation value of Eq. (46) in this state. Since the beam-splitter scattering matrix does not include spin-flip processes, this expectation value can be expressed in terms of the shot noise for the unrotated singlet $\left(|\uparrow \downarrow\rangle_{a a, z}-|\downarrow \uparrow\rangle_{a a, z}\right) / 2$ and unentangled triplets $|\uparrow \uparrow\rangle_{a a, z}$, $|\downarrow \downarrow\rangle_{a a, z}$

$$
S_{33}^{\Psi_{2}}\left(\theta_{\mathrm{SO}}\right)=\frac{1}{2} \cos ^{2}\left(\theta_{\mathrm{SO}} / 2\right)\left[\widetilde{S}_{33 ; a a}^{T_{u_{\uparrow}}}+\widetilde{S}_{33 ; a a}^{T_{u_{\downarrow} ; z}}\right]+\sin ^{2}\left(\theta_{\mathrm{SO}} / 2\right) \widetilde{S}_{33 ; a a}^{S},
$$

where

$$
\tilde{S}_{33 ; a a}^{S}=\frac{2 e^{2}}{h \nu} T(1-T)\left(1+\delta_{\varepsilon_{1}, \varepsilon_{2}}\right)
$$

and

$$
\widetilde{S}_{33 ; a a}^{T_{\uparrow}}=\widetilde{S}_{33 ; a a}^{T_{u_{1}}}=\frac{2 e^{2}}{h \nu} T(1-T)\left(1-\delta_{\varepsilon_{1}, \varepsilon_{2}}\right) .
$$

Note that $\widetilde{S}_{33 ; a a}^{S}$ and $\widetilde{S}_{33 ; a a}^{T_{u \sigma z}}$ denote the shot noise for the bare singlet and triplet states, respectively. ${ }^{6}$ Equations (75) and (76) represent the noise expectation value for the bare singlet and triplet states ${ }^{6}$ and follows from Eqs. (61) and (62) with $\theta_{\mathrm{SO}}=0$. Substituting the Eqs. (75) and (76) into (74), we find

$$
S_{33}^{\Psi_{2}}\left(\theta_{\mathrm{SO}}\right)=\frac{2 e^{2}}{h \nu} T(1-T)\left[1-\cos \left(\theta_{\mathrm{SO}}\right) \delta_{\varepsilon_{1}, \varepsilon_{2}}\right] .
$$

Similarly, 


$$
S_{33}^{\Psi_{3}}\left(\theta_{\mathrm{SO}}\right)=\frac{2 e^{2}}{h \nu} T(1-T)\left(1-\delta_{\varepsilon_{1}, \varepsilon_{2}}\right) .
$$

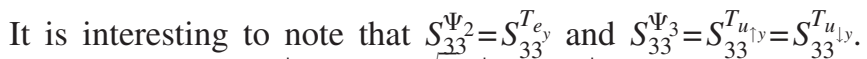
This follows from $\left|T_{e_{y}}\right\rangle=(1 / \sqrt{2})\left(|\uparrow \downarrow\rangle_{y}-|\downarrow \uparrow\rangle_{y}\right)$ being equal to $\left|\Psi_{2}\right\rangle$ and $\left|\Psi_{3}\right\rangle=\left|T_{u_{\uparrow}}\right\rangle$ for the quantization axis along $z$.

d. Local Zeeman and spin-orbit-induced rotations. It is interesting to note that the shot noise modulation induced by the local SO interaction in the uncoupled-channel case discussed above is formally identical to that due to a local Zeeman interaction in lead 1 (e.g., due to a local magnetic field or a distinct $g$ factors in lead 1). For a local Zeeman interaction, $\theta_{\mathrm{SO}}$ should be equal to $g \mu_{B} B L / v$, i.e., the phase acquired by the electron upon traversing, with velocity $v$, the length $L$ of the Zeeman-active region. Note, however, that this formal correspondence between local Zeeman and SO interactions holds only for the uncoupled-channel case (or in a strictly single-channel lead) and when magnetic-fieldinduced orbital effects are neglected.

e. Shot noise for electron pairs: physical picture. Before moving over to the more elaborate case with coupled channels, we provide here a simple picture for the shot noise results we obtained for the singlet and triplets. Let us first consider the case with no spin orbit $\left(\theta_{\mathrm{SO}}=0\right)$ and one orbital channel. Our formulas yield bunching for singlet and antibunching for the triplets, as previously found in Ref. 6. This bunching and antibunching behavior for the singlet and triplets pairs can be readily understood if we write out the pair wave functions in the outgoing leads $(3,4)$ similarly to Ref. 65. The injected singlet and entangled triplets states $\left|S / T_{e_{2}}\right\rangle$ $=\left(a_{1, \uparrow}^{\dagger} a_{2, \downarrow}^{\dagger} \bar{\mp} a_{1, \downarrow}^{\dagger} a_{2, \uparrow}^{\dagger}\right)|0\rangle / \sqrt{2}$ and $\left|T_{u_{\sigma, i}}\right\rangle=a_{1 \sigma}^{\dagger} a_{2 \sigma}^{\dagger}|0\rangle$ after traversing the beam splitter evolve into

$$
\begin{aligned}
|S\rangle \rightarrow & \sqrt{2} r t b_{3, \uparrow}^{\dagger} b_{3, \downarrow}^{\dagger}|0\rangle+\sqrt{2} r t b_{4, \uparrow}^{\dagger} b_{4, \downarrow}^{\dagger}|0\rangle \\
& +\frac{1}{\sqrt{2}}\left(r^{2}+t^{2}\right)\left(b_{3, \uparrow}^{\dagger} b_{4, \downarrow}^{\dagger}-b_{3, \downarrow}^{\dagger} b_{4, \uparrow}^{\dagger}\right)|0\rangle, \\
\left|T_{e_{z}}\right\rangle \rightarrow & \frac{1}{\sqrt{2}}\left(r^{2}-t^{2}\right)\left(b_{3, \uparrow}^{\dagger} b_{4, \downarrow}^{\dagger}+b_{3, \downarrow}^{\dagger} b_{4, \uparrow}^{\dagger}\right)|0\rangle,
\end{aligned}
$$

and

$$
\left|T_{u_{\sigma, z}}\right\rangle \rightarrow\left(t^{2}-r^{2}\right) b_{4, \sigma}^{\dagger} b_{3, \sigma}^{\dagger},
$$

where the $b_{i, \sigma}^{\dagger}$ 's $(i=3,4)$ represent the creation operators in the outgoing leads, directly related to the $a_{i, \sigma}^{\dagger}$ 's via the beamsplitter scattering matrix $\mathbf{s}$ (note that here the particular quantization axis is irrelevant; this is not true in the presence of the spin-orbit interaction as described below). Interestingly, the incoming electrons in the triplet states in leads 1 and 2 have zero probability of emerging in the same outgoing lead (note that $\left|r^{2}-t^{2}\right|=1$ due to $\mathbf{s}^{\dagger} \mathbf{s}=\mathbf{1}$ ). This is the (full) antibunching we mentioned above and it results in zero shot noise for the injected triplet pairs. Physically, this vanishing of the shot noise means that there is no randomness in the electron flow in the outgoing leads: each electron of the incoming triplet pairs goes-with unity probability-to dis- tinct outgoing leads. For the singlet pairs, on the other hand, the probability for the two electrons to emerge in the same outgoing arm is not zero as for the triplets. This probability is larger by a factor of two than the classical value $|r|^{2}|t|^{2}$. Hence the incoming electrons in a singlet pair tend to bunch, i.e., to go to the same outgoing leads. Full bunching occurs only for the particular case of a 50:50 beam splitter. In this case we have $r= \pm i t$ and opposite spins have a $1 / 2$ probability to emerge in one of the leads 3 or 4 . This randomness in the electron flow in the outgoing leads increases the shot noise as compared to both the triplet and the classical case.

Note that the bunching and antibunching behaviors described above follow from the stringent requirement for antisymmetry (Pauli's principle) of the total wave function of the electron pair. In an unentangled triplet, for instance, the spin part of the wave function is symmetric thus forcing its spatial component to be antisymmetric which results in a strong correlation between the electrons in the triplet pair, i.e., they avoid each other by always going into distinct outgoing leads. The same is true for the entangled triplet pair. This correlation reduces the shot noise, in agreement with the classical notion of shot noise suppression which occurs when the "discreteness" of the electron flow is reduced. For the singlet, on the other hand, the spin part of the pair wave function is antisymmetric which makes the spatial part symmetric. The electrons in a singlet pair can overlap freely in space and hence are less correlated than the triplet case thus giving rise to nonzero shot noise. Interestingly, the shot noise for the singlet is even larger than the classical analog (i.e., a distinguishable pair injected into leads 1 and 2). This also agrees with the classical notion of shot noise as due to the discreteness of the charge flow: because the spatial part of the singlet pair is maximally "uncorrelated" ("negative correlation") the discreteness of the electron charge is larger than the classical case (the singlet electrons can lie completely on top of each other in real space) thus yielding a larger shot noise.

In the presence of spin-orbit coupling, the above ideas can be straightforwardly generalized. In this case the singlet and triplets injected into single-channel leads evolve into

$$
\begin{aligned}
|S\rangle \rightarrow & \sqrt{2} r t \cos \left(\theta_{\mathrm{SO}} / 2\right) b_{3, \uparrow}^{\dagger} b_{3, \downarrow}^{\dagger}|0\rangle+\sqrt{2} t r \cos \left(\theta_{\mathrm{SO}} / 2\right) b_{4, \uparrow}^{\dagger} b_{4, \downarrow}^{\dagger}|0\rangle \\
+ & \left(t^{2}+r^{2}\right) \cos \left(\theta_{\mathrm{SO}} / 2\right) \frac{1}{\sqrt{2}}\left(b_{3, \uparrow}^{\dagger} b_{4, \downarrow}^{\dagger}-b_{3, \downarrow}^{\dagger} b_{4, \uparrow}^{\dagger}\right)|0\rangle+\left(t^{2}\right. \\
- & \left.r^{2}\right) \sin \left(\theta_{\mathrm{SO}} / 2\right) \frac{1}{\sqrt{2}}\left(b_{3, \downarrow}^{\dagger} b_{4, \downarrow}^{\dagger}+b_{3, \uparrow}^{\dagger} b_{4, \uparrow}^{\dagger}\right)|0\rangle, \\
\left|T_{e_{z}}\right\rangle \rightarrow & \left(r^{2}-t^{2}\right) \cos \left(\theta_{\mathrm{SO}} / 2\right) \frac{1}{\sqrt{2}}\left(b_{3, \uparrow}^{\dagger} b_{4, \downarrow}^{\dagger}+b_{3, \downarrow}^{\dagger} b_{4, \uparrow}^{\dagger}\right)|0\rangle \\
& +\left(t^{2}-r^{2}\right) \sin \left(\theta_{\mathrm{SO}} / 2\right) \frac{1}{\sqrt{2}}\left(b_{3, \downarrow}^{\dagger} b_{4, \downarrow}^{\dagger}-b_{3, \uparrow}^{\dagger} b_{4, \uparrow}^{\dagger}\right)|0\rangle,
\end{aligned}
$$

and 


$$
\begin{aligned}
\left|T_{u_{\uparrow}, z}\right\rangle \rightarrow & \cos \left(\theta_{\mathrm{SO}} / 2\right)\left(t^{2}-r^{2}\right) b_{4, \uparrow}^{\dagger} b_{3, \uparrow}^{\dagger}|0\rangle \\
& +\sin \left(\theta_{\mathrm{SO}} / 2\right) r t\left(b_{3, \uparrow}^{\dagger} b_{3, \downarrow}^{\dagger}+b_{4, \uparrow}^{\dagger} b_{4, \downarrow}^{\dagger}\right)|0\rangle \\
& +\sin \left(\theta_{\mathrm{SO}} / 2\right) t^{2} b_{3, \uparrow}^{\dagger} b_{4, \downarrow}^{\dagger}|0\rangle-\sin \left(\theta_{\mathrm{SO}} / 2\right) r^{2} b_{3, \downarrow}^{\dagger} b_{4, \uparrow}^{\dagger}|0\rangle .
\end{aligned}
$$

The unentangled triplet $\left|T_{u_{1}, z}\right\rangle$ evolves similarly to $\left|T_{u_{\uparrow}, z}\right\rangle$. Due to the continuous spin rotation induced by the SO coupling, intermediate degrees of bunching and antibunching are possible as the modulation angle $\theta_{\text {SO }}$ is varied. Note that a variety of entangled and unentangled electron pairs (along distinct quantization axes as well) can be generated according to the above states by sending electron pairs through a SO active region. See also Ref. 66 for entanglement generation using a Mach-Zehnder interferometer.

\section{Coupled-channel case}

Here we assume that electron pairs are injected into channel $a$ of leads 1 and 2 with energies near the crossing of the bands at $k_{c}$, Figs. 1(b) and 2. The case with injection into both channels $a$ and $b$ is discussed at the end of this section. General expressions similar to Eqs. (52) and (53) can, in principle, be derived for this case. Here, however, we generalize the approach outlined at the end of the previous section to the the case of two SO-coupled channels, which is both simpler and more intuitive. An additional heuristic derivation of the noise properties using simple number operators is given in the Appendix C 1.

a. Quantization axis along $z$. The idea is essentially the same as before: for instance, an injected unentangled spin-up triplet along the $z$ direction, with one electron in channel $a$ of lead 1 an the other in channel $a$ of lead 2 evolves into [Eq. (39)]

$$
\begin{aligned}
\left|T_{u_{\uparrow, z}}\right\rangle^{L}= & \frac{1}{2}\left[\cos \left(\theta_{d} / 2\right) e^{-i \theta_{\mathrm{SO}^{2}} / 2}+e^{i \theta_{\mathrm{SO}} / 2}\right]|\uparrow \uparrow\rangle_{a a, z} \\
& +\frac{1}{2} \xi\left[\cos \left(\theta_{d} / 2\right) e^{-i \theta_{\mathrm{SO}} / 2}-e^{i \theta_{\mathrm{SO}} / 2}\right]|\downarrow \uparrow\rangle_{a a, z} \\
& -\frac{1}{2} i \sin \left(\theta_{d} / 2\right) e^{-i \theta_{\mathrm{SO}^{\prime}} / 2}|\uparrow \uparrow\rangle_{b a, z} \\
& +\frac{1}{2} i \xi \sin \left(\theta_{d} / 2\right) e^{-i \theta_{\mathrm{SO}} / 2}|\downarrow \uparrow\rangle_{b a, z},
\end{aligned}
$$

upon traversing the SO region in lead 1 (we dropped an overall phase $\left.e^{i\left(k_{c}+k_{\mathrm{SO}}\right) L}\right)$. Similarly to the uncoupled-channel case, we note that only the portion of the electron pair going through lead 1 is subject to the SO effect. Note that the SO induced interband coupling in lead 1 makes the injected states initially in channel $a$ leak into channel $b$. The expectation value of the noise in the state $\left|T_{u_{\uparrow, z}}\right\rangle^{L}$ is

$$
\begin{aligned}
S_{33}^{T_{u_{\uparrow}, z}\left(\theta_{\mathrm{SO}}, \theta_{d}\right)=} & \frac{1}{4}\left|\cos \left(\theta_{d} / 2\right) e^{-i \theta_{\mathrm{SO}} / 2}+e^{i \theta_{\mathrm{SO}} / 2}\right|^{2} \widetilde{S}_{33 ; a a}^{T_{u_{\uparrow}, z}} \\
& +\frac{|\xi|^{2}}{4}\left|\cos \left(\theta_{d} / 2\right) e^{-i \theta_{\mathrm{SO}} / 2}-e^{i \theta_{\mathrm{SO}} / 2}\right|^{2} \widetilde{S}_{33 ; a a}^{\downarrow \uparrow, z} \\
& +\frac{1}{4} \sin ^{2}\left(\theta_{d} / 2\right) \widetilde{S}_{33 ; b a}^{T_{u_{\uparrow}}}+\frac{1}{4} \sin ^{2}\left(\theta_{d} / 2\right) \tilde{S}_{33 ; b a}^{\downarrow \uparrow, z},
\end{aligned}
$$

where $\widetilde{S}_{33 ; b a}^{\downarrow \uparrow, z}$ denotes the noise expectation value, Eq. (46), for a pair with a spin-down electron in channel $b$ of lead 1 and a spin-up electron in channel $a$ of lead $2,|\downarrow \uparrow\rangle_{b a, z}$. Similarly, $\tilde{S}_{33 ; a a}^{\downarrow \uparrow, z}$ corresponds to the two opposite spin electrons in the channel $a$ of the respective lead. The noise contribution of the pair state $|\downarrow \uparrow\rangle_{a a, z}$ can be determined from Eq. (62) by setting $\theta_{\mathrm{SO}}=\pi$ which makes $\left|T_{u_{\uparrow, z}}\right\rangle^{L}=-|\downarrow \uparrow\rangle_{a a, z}$, see Eq. (71),

$$
\tilde{S}_{33 ; a a}^{\downarrow \uparrow, z}=\frac{2 e^{2}}{h \nu} T(1-T) \text {. }
$$

Note that the pair state $|\downarrow \uparrow\rangle_{a a, z}$ is distinguishable (spin down in lead 1 and spin up in lead 2 , both in channel $a$ ), and therefore yields the classical shot noise. Similarly, for the distinguishable state $|\downarrow \uparrow\rangle_{b a, z}$ we should have $\widetilde{S}_{33 ; b a}^{\downarrow \uparrow, z}=\widetilde{S}_{33 ; a a}^{\downarrow \uparrow, z}$. Hence we find

$$
\begin{aligned}
& S_{33}^{T_{u_{\uparrow, z}}}\left(\theta_{\mathrm{SO}}, \theta_{d}\right)=\frac{e^{2}}{h \nu} T(1-T)\left[1+\sin ^{2}\left(\theta_{d} / 2\right) / 2 \delta_{\varepsilon_{1}, \varepsilon_{2}}\right. \\
& \left.-\cos \left(\theta_{d} / 2\right) \cos \left(\theta_{\mathrm{SO}}\right) \delta_{\varepsilon_{1}, \varepsilon_{2}}\right]
\end{aligned}
$$

and, similarly, $S_{33^{1, z}}^{T_{u}}\left(\theta_{\mathrm{SO}}, \theta_{d}\right)=S_{33_{\uparrow, z}}^{T_{u}}\left(\theta_{\mathrm{SO}}, \theta_{d}\right)$. The noise for the entangled triplet and the singlet are, respectively,

$$
S_{33}^{T} T_{z}\left(\theta_{\mathrm{SO}}, \theta_{d}\right)=\frac{2 e^{2}}{h \nu} T(1-T)\left[1-\frac{1}{2}\left[\cos ^{2}\left(\theta_{d} / 2\right)+1\right] \delta_{\varepsilon_{1}, \varepsilon_{2}}\right]
$$

and

$$
S_{33}^{S}\left(\theta_{\mathrm{SO}}, \theta_{d}\right)=\frac{2 e^{2}}{h \nu} T(1-T)\left[1+\cos \left(\theta_{d} / 2\right) \cos \left(\theta_{\mathrm{SO}}\right) \delta_{\varepsilon_{1}, \varepsilon_{2}}\right]
$$

b. Quantization axis along $y$. We can again straightforwardly find all the shot noise expressions for the quantization axis along $y$ by following the same procedure as above. However, here a spin up (along the $y$ direction) electron injected into the channel $a$ of lead 1 evolves into

$$
\begin{aligned}
|\uparrow\rangle_{a, y}^{L}= & \frac{1}{2 \sqrt{2}}\left\{\left[\left(1+i \xi^{*}\right) \cos \left(\theta_{d} / 2\right) e^{-i \theta_{\mathrm{SO}} / 2}+\left(1-i \xi^{*}\right) e^{i \theta_{\mathrm{SO}} / 2}\right]|\uparrow\rangle_{a, z}\right. \\
& +\left[(\xi+i) \cos \left(\theta_{d} / 2\right) e^{-i \theta_{\mathrm{SO}^{\prime}} / 2}+(i-\xi) e^{i \theta_{\mathrm{SO}} / 2}\right]|\downarrow\rangle_{a, z} \\
& +\left(\xi^{*}-i\right) \sin \left(\theta_{d} / 2\right) e^{-i \theta_{\mathrm{SO}} / 2}|\uparrow\rangle_{b, z} \\
& \left.+(i \xi-1) \sin \left(\theta_{d} / 2\right) e^{-i \theta_{\mathrm{SO}^{\prime}} / 2}|\downarrow\rangle_{b, z}\right\}
\end{aligned}
$$


upon traversing the SO region in lead 1, while a spin down electron (along the $y$ ) evolves according to

$$
\begin{aligned}
|\downarrow\rangle_{a, y}^{L}= & \frac{1}{2 \sqrt{2}}\left\{\left[\left(1-i \xi^{*}\right) \cos \left(\theta_{d} / 2\right) e^{-i \theta_{\mathrm{SO}} / 2}+\left(1+i \xi^{*}\right) e^{i \theta_{\mathrm{SO}} / 2}\right]|\uparrow\rangle_{a, z}\right. \\
& +\left[(\xi-i) \cos \left(\theta_{d} / 2\right) e^{-i \theta_{\mathrm{SO}} / 2}\right. \\
& \left.-(i+\xi) e^{i \theta_{\mathrm{SO}} / 2}\right]|\downarrow\rangle_{a, z}-\left(i+\xi^{*}\right) \sin \left(\theta_{d} / 2\right) e^{-i \theta_{\mathrm{SO}} / 2}|\uparrow\rangle_{b, z} \\
& \left.+(i \xi+1) \sin \left(\theta_{d} / 2\right) e^{-i \theta_{\mathrm{SO}} / 2}|\downarrow\rangle_{b, z}\right\} .
\end{aligned}
$$

For simplicity, we consider below the case with only the Rashba coupling, i.e., $\alpha \neq 0$ and $\beta=0$. In this case, the above equations simplify to

$$
|\uparrow\rangle_{a, y}^{L}=\exp \left(i \theta_{R} / 2\right)|\uparrow\rangle_{a, y}
$$

and

$$
|\downarrow\rangle_{a, y}^{L}=\exp \left(-i \theta_{R} / 2\right)\left[\cos \left(\theta_{d} / 2\right)|\downarrow\rangle_{a, y}-i \sin \left(\theta_{d} / 2\right)|\uparrow\rangle_{b, y}\right],
$$

where $\theta_{\mathrm{SO}} \rightarrow \theta_{R}=2 m \alpha L / \hbar^{2}$ and $|\uparrow\rangle_{a, y}$ and $|\downarrow\rangle_{a, y}$ are the eigenspinors of $\sigma_{y}$ in channel $a$ (similar definitions hold for channel $b$ ). Note that the spin-up and spin-down states in channel $a$ of lead 1 evolve quite differently. In particular, the spin-up state remains in channel $a$ and only picks up a overal phase, while the spin-down one acquires a spin-up component in channel $b$. This distinct evolution of $|\uparrow\rangle_{a, y}^{L}$ and $|\downarrow\rangle_{a, y}^{L}$ can be understood if we recall that the incoming electron state $|\uparrow\rangle_{a, y}$ is essentially an eigenstate of the system at $x=0$ (far away from any level crossing) and hence evolves as such, as the electron traverses the SO region. In contrast, the state $|\downarrow\rangle_{a, y}^{L}$ results from the incoming spin-down state in channel $a$ being injected at the level crossing corresponding to the states $|\downarrow\rangle_{a, y}$ and $|\uparrow\rangle_{b, y}$ and thus evolves as a coherent superposition of these two states along lead 1. Hence, $|\uparrow\rangle_{a, y}^{L}$ is an eigenstate of our Hamiltonian in the approximation we considered in Eq. (26), while $|\downarrow\rangle_{a, y}^{L}$ is not, due to the weak coupling between channels $a$ and $b$ at the crossing.

From the states (93) and (94) we can construct the electron pairs (entangled or not) that we are interested in, e.g., the singlet and the triplet states along the $y$ direction. Let us consider a pair injected into only the channel $a$ of leads 1 and 2. For the spin-up triplet we find

$$
\left|T_{u_{\uparrow, y}}\right\rangle^{L}=\exp \left(i \theta_{R} / 2\right)|\uparrow \uparrow\rangle_{a a, y},
$$

while for the spin down

$$
\left|T_{u_{\downarrow, y}}\right\rangle^{L}=\exp \left(-i \theta_{R} / 2\right)\left[\cos \left(\theta_{d} / 2\right)|\downarrow \downarrow\rangle_{a a, y}-i \sin \left(\theta_{d} / 2\right)|\uparrow \downarrow\rangle_{b a, y}\right] .
$$

The entangled triplet and singlet are found to be

$$
\begin{aligned}
\left|S / T_{e_{y}}\right\rangle^{L}= & \frac{1}{\sqrt{2}}\left[\exp \left(i \theta_{R} / 2\right)|\uparrow \downarrow\rangle_{a a, y} \mp \exp \left(-i \theta_{R} / 2\right) \cos \left(\theta_{d} / 2\right)\right. \\
& \left.\times|\downarrow \uparrow\rangle_{b a, y}\right] \pm \frac{1}{\sqrt{2}} i \exp \left(-i \theta_{R} / 2\right)|\uparrow \uparrow\rangle_{b a, y}
\end{aligned}
$$

where the upper (lower) sign corresponds to the singlet (triplet) state. The noise expression corresponding to the above states are

$$
\begin{gathered}
S_{33^{3}, \nu}^{T_{u_{\uparrow}}}\left(\theta_{d}\right)=\frac{2 e^{2}}{h \nu} T(1-T)\left(1-\delta_{\varepsilon_{1}, \varepsilon_{2}}\right), \\
S_{33^{\prime}}^{T_{u_{1}, \nu}}\left(\theta_{d}\right)=\frac{2 e^{2}}{h \nu} T(1-T)\left[1-\cos ^{2}\left(\theta_{d} / 2\right) \delta_{\varepsilon_{1}, \varepsilon_{2}}\right],
\end{gathered}
$$

and

$$
S_{33}^{S / T} e_{e_{y}}\left(\theta_{R}, \theta_{d}\right)=\frac{2 e^{2}}{h \nu} T(1-T)\left[1 \pm \cos \left(\theta_{d} / 2\right) \cos \left(\theta_{R}\right) \delta_{\varepsilon_{1}, \varepsilon_{2}}\right] .
$$

Interestingly, the above results show that by measuring the noise in lead 3 along the $y$ quantization axis one can distinguish all the electron pairs as they display distinct noise for non-zero SO induced interchannel mixing angles $\left(\theta_{d}\right.$ $\neq 0)$. Figure 3 shows the reduced Fano factor $f$ $=S_{33} /\left[2 e^{2} T(1-T) / h \nu\right]$ for the singlet and triplets along the $y$ axis as a function of $\theta_{\mathrm{SO}}$ and $\theta_{d}$. It clearly shows that the singlet and triplets display distinct shot noise in a wide range of angles. Note that this result holds only for incoming electron pairs injected initially into channel $a$ and with energies near the crossing.

\section{Effect of backscattering in the Rashba lead}

Here we examine how the presence of backscattering in the lead containing the spin-orbit interaction (lead 1) affects our results for the current fluctuations. A small backscattering can indeed be produced by the small band offset between the regions of the lead with and without the Rashba interaction; see Appendix B where we calculate explicitly the transmission and reflection coefficients for a model quantum wire with spin-orbit interaction of both the Dresselhaus and Rahsba types. The situation is depicted in Fig. 4(a).

We emphasize that here the "left" leads $(1,3)$ and "right" leads $(2,4)$ are not equivalent anymore because of the presence of the spin-orbit region in lead 1. This, as we shall see below, yields that $S_{33} \neq S_{44}$. The case in which backscattering is present in both leads 1 and 2 was first addressed in Ref. 67, and is discussed in the Appendix C 1 within a simple heuristic picture [see Fig. 4(b)]. For simplicity, we consider here only the case of uncoupled bands (Sec. II C).

We take into account the backscattering by adding a tunnel barrier with reflection amplitude $a$ to lead 1, described by the scattering matrix 

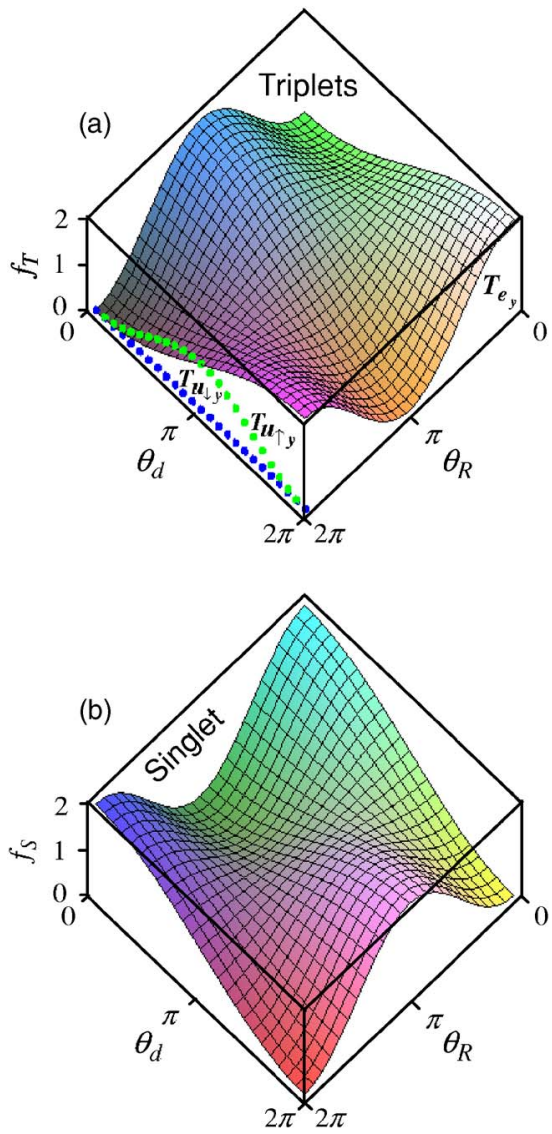

FIG. 3. (Color online) Fano factors as a function of $\theta_{R}$ and $\theta_{d}$ for triplet (a) and singlet (b) pairs (defined along the $y$ axis) with injection energies near the channel anticrossings. We assume the injected pairs to be initially in channel $a$ of the non-Rashba region in lead 1. In addition to the usual Rashba-induced spin rotation $\theta_{R}$, the spin-orbit interaction induces a further modulation on the Fano factors via the coherent transfer of electrons between the channels (mixing angle $\theta_{d}$ ). Interestingly, the triplets display distinct Rashba SO modulations: the unentangled spin-up pair $T_{u_{\uparrow} y}$ is not sensitive to spin-orbit effects and shows full antibunching, the unentangled spin-down triplet $T_{u, y}$ depends on only $\theta_{d}$ and oscillates between the full anti-bunching and the classical value for distinguishable pairs, the entangled triplet $T_{e_{y}}$ displays sizable oscillations between full bunching and antibunching as $\theta_{d}$ and $\theta_{R}$ are varied. For the sake of clarity, we have omitted in (a) the $\theta_{R}$ dependence of Fano factors corresponding to $T_{u_{\uparrow} y}$ and $T_{u_{1} y}$.

$$
\mathbf{S}_{\mathrm{back}}=\left(\begin{array}{cc}
a & b \\
b & a
\end{array}\right)
$$

In order to combine $\mathbf{s}_{\text {back }}$ with the beam-splitter scattering matrix s [Eq. (47)], we match the coefficients of the wave function going out of $\mathbf{s}_{\text {back }}$ with the corresponding ones going in $\mathbf{s}$, and solve the resulting system of equations eliminating the intermediate coefficients. ${ }^{68}$ We obtain the modified scattering matrix
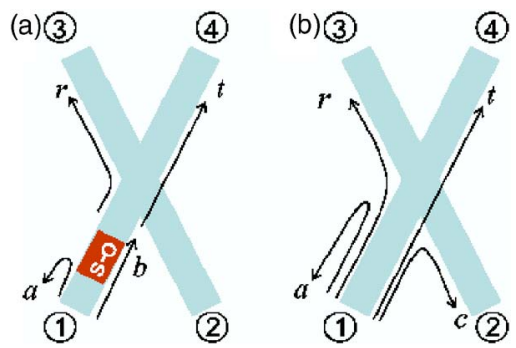

FIG. 4. (Color online) Two cases for a beam splitter with backscattering. (a) Backscattering in arm 1 only, induced by the SO region, with $a$ the backscattering amplitude, and $r$ and $t$ the original amplitudes of the beam splitter. (b) Backscattering present in all leads (amplitude $a$ ), with also cross-backscattering (c). Here $r$ and $t$ are the modified amplitudes satisfying the normalization $|a|^{2}+|c|^{2}$ $+|r|^{2}+|t|^{2}=1$. This case will be discussed in the Appendix C 1 .

$$
\mathbf{s}^{\prime}=\left(\begin{array}{cccc}
a & 0 & b r & b t \\
0 & 0 & t & r \\
b r & t & a r^{2} & a r t \\
b t & r & a r t & a t^{2}
\end{array}\right) .
$$

The probability $A=|a|^{2}$ of backscattering in lead 1 renormalizes the transmission probabilities $R=|r|^{2}$ and $T=|t|^{2}$ by the factor $|b|^{2}=1-A$. It is interesting to note that the constraints of unitarity introduce some backscattering in the leads 3 and 4. These correspond to the possibility for particles injected into leads 3 or 4 to scatter back into leads 3 and 4 after being reflected by the tunnel barrier in lead 1 . We note that the probabilities $R$ and $T$ are the ones of the original beam splitter, and as such satisfy the normalization condition $R=1-T$.

We proceed as before and introduce the spin rotation due to the Rashba coupling by multiplying $s_{13}$ and $s_{14}$ by $\mathbf{U}_{\mathrm{SO}}$, Eqs. (48) and (49). The spin rotation has no effect on $s_{11}$ even if the backscattering occurs after the Rashba region, because the spin of a particle with reversed momentum rotates in the reversed direction. We can now use the general formulas Eqs. (52) and (53) to evaluate the autocorrelation noise in lead 3 . Introducing the renormalized probability $R^{\prime}=R(1-A)$, we find

$$
S_{33}^{S}\left(\theta_{\mathrm{SO}}\right)=\frac{e^{2}}{h \nu}\left[T R+R^{\prime}\left(1-R^{\prime}\right)+2 T R^{\prime} \cos \left(\theta_{\mathrm{SO}}\right) \delta_{\epsilon_{1}, \epsilon_{2}}\right]
$$

$$
\begin{gathered}
S_{33_{z}}^{T_{e_{z}}}=\frac{e^{2}}{h \nu}\left[T R+R^{\prime}\left(1-R^{\prime}\right)-2 T R^{\prime} \delta_{\epsilon_{1}, \epsilon_{2}}\right], \\
S_{33}^{T_{u_{\uparrow, z}}\left(\theta_{\mathrm{SO}}\right)=} \frac{e^{2}}{h \nu}\left[T R+R^{\prime}\left(1-R^{\prime}\right)-2 T R^{\prime} \cos ^{2}\left(\theta_{\mathrm{SO}} / 2\right) \delta_{\epsilon_{1}, \epsilon_{2}}\right],
\end{gathered}
$$

with the average current in lead 3

$$
I_{3}=V \frac{e^{2}}{h}(1-A R)
$$



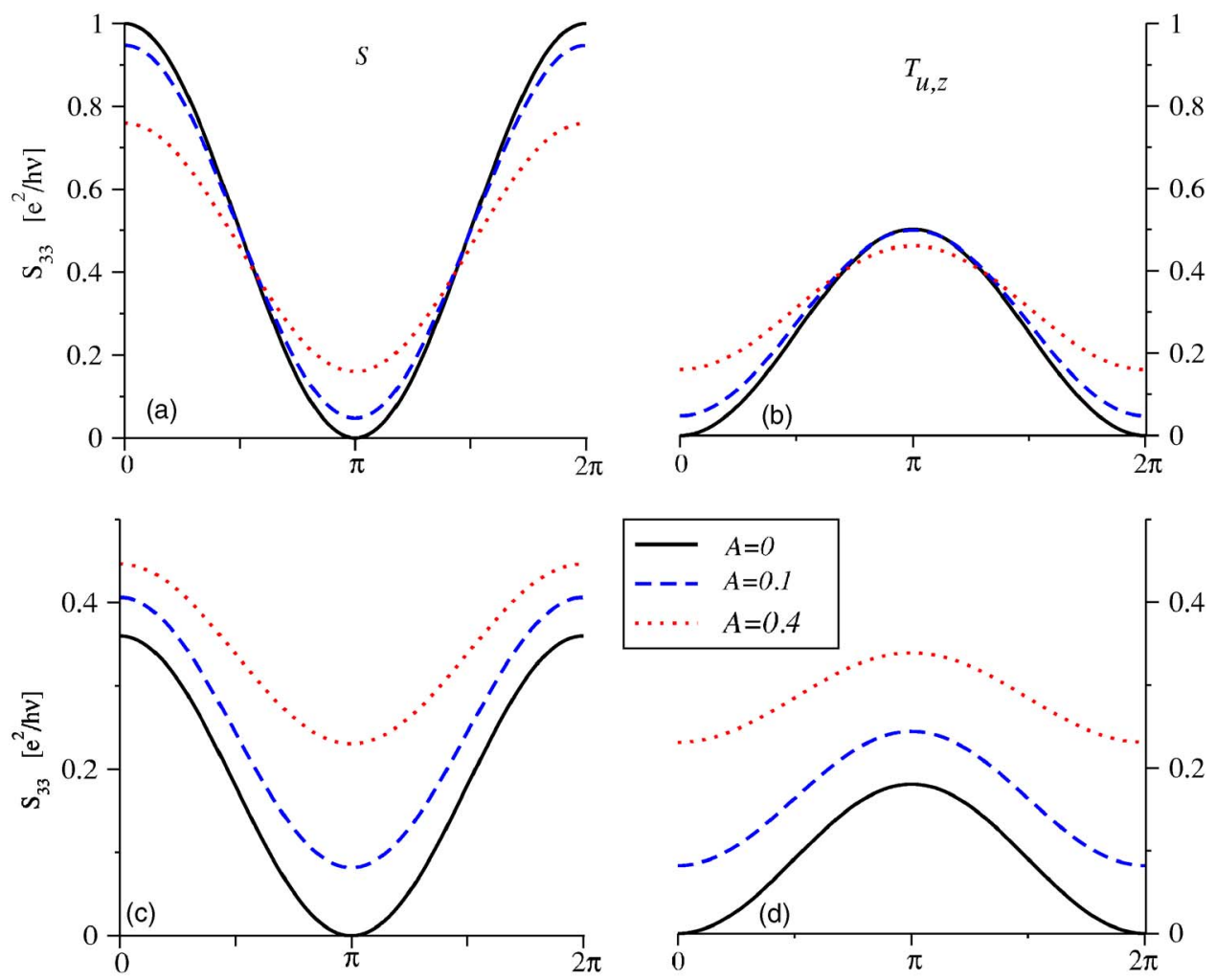

FIG. 5. (Color online) Noise in the presence of backscattering in the lead with SO interaction, for backscattering probabilities given by $A=0,0.1$, and 0.4 . The transmission probabilities are $R=1-T$ $=0.5$ (a), (b); $R=0.9$ (c), (d); and $R=0.1$ (e), (f). The left (right) column shows the case of the singlet $S$ (unentangled triplet $T_{u, z}$ ) with $\epsilon_{1}=\epsilon_{2}$.

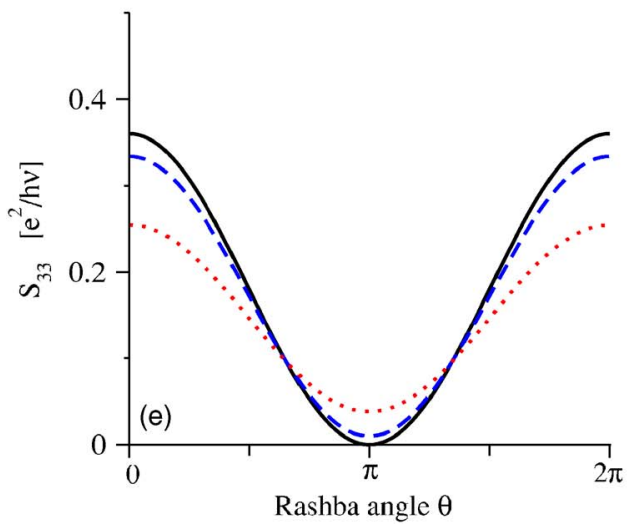

The factor $2 T R$ found in the previous case without backscattering, Eqs. (60)-(62), splits into two contributions, $T R$ $+R^{\prime}\left(1-R^{\prime}\right)$ with the renormalized probability $R^{\prime}$. The backscattering adds a contribution related to the partition noise created by the tunneling barrier. Partition noise (shot noise) corresponds to fluctuations arising from the fact the barrier splits randomly the incident electron flow into transmitted and backscattered flows. On the other hand, the backscattering reduces the transmission probability $R^{\prime}$ for electrons injected in lead 1 , and therefore can decrease the "beamsplitter noise" proportional to $R(1-R)$. For instance, the noise for entangled triplets with same energies is increased from zero (in the absence of backscattering) to a finite value. Similarly, the noise for electrons with different energies is increased in the range $0 \leqslant A \leqslant \max \{2-1 / T, 0\}$, with a maximal increase of $(T-1 / 2)^{2}\left(e^{2} / h \nu\right)$. However, the maximal value for the noise (obtained in the case of singlets with $\epsilon_{1}$ $=\epsilon_{2}$ and $\left.\theta_{\mathrm{SO}}=2 n \pi, n \in \mathbb{Z}\right)$ is not changed, as it corresponds to the maximal value reached in the case of bunching of bosonic-like particles. In the case of spin pairs injected along the $O y$ axis, the result for the singlet remains the same, while $S_{33}^{T_{u, y}}=S_{33}^{T_{e}, z}$ and $S_{33}^{T_{e, y}}(\theta)=S_{33}^{S}\left(\pi-\theta_{\mathrm{SO}}\right)$.

Figure 5 shows the autocorrelation noise $S_{33}$ in the presence of backscattering in lead 1, of the singlet $S$ and for the unentangled triplet $T_{u, z}$ in the case of equal energies $\epsilon_{1}=\epsilon_{2}$. We recall that the noise of the entangled triplet $T_{e, z}$ is independent of $\theta_{\mathrm{SO}}$, as are $S$ and $T_{u, z}$ for different energies. We see that the backscattering can either increase or decrease the noise, depending on the rotation angle $\theta_{\mathrm{SO}}$ as well as the beam-splitter properties (defined by $R$ ). However, in all cases the backscattering reduces the visibility (amplitude) of the $\theta_{\text {SO }}$ oscillations. In the absence of backscattering $(A=0)$, the bunching of singlets for $\theta_{\mathrm{SO}}=0$ is maximal $\left(S_{33}=e^{2} / h \nu\right)$ for a symmetric beam splitter (i.e., $T=R=0.5$ ); this comes from the fact that the randomness is maximal in this configuration. In the presence of backscattering, the effective transmission is decreased via $R \rightarrow R^{\prime}(1-A)$, so that as one moves away from this symmetric point, the randomness of the beam- 
splitter is reduced, and the noise is suppressed. Similarly, the perfect antibunching seen at $\theta_{\mathrm{SO}}=\pi$ (and at $\theta_{\mathrm{SO}}=0$ for the triplets) no longer happens because of the residual partition noise related to the backscattering and the noise is given by $S_{33}^{S}\left(\theta_{\mathrm{SO}}=\pi\right)=S_{33}^{T_{u_{\uparrow}, z}}\left(\theta_{\mathrm{SO}}=0\right)=S_{33^{2}}^{T_{e}}\left(\epsilon_{1}=\epsilon_{2}\right)=\left(e^{2} / h \nu\right) A R(1-A R)$ (see Appendix $\mathrm{C}$, where we present a heuristic derivation of these results via simple calculations of expectation values of number operators).

In the case of an asymmetric beam-splitter with a large value of $R>3 /(4-A)$ (e.g., $R=0.9)$, the noise for the singlets is increased by the backscattering for all $\theta_{\mathrm{SO}}$. For $\theta_{\mathrm{SO}}=0$, one can understand this by noting that the backscattering reduces the value of $R^{\prime}$ which therefore gets closer to the symmetric value of 0.5 related to the maximal noise. On the other, in the case of small $R$ (e.g., $R=0.1$ ), the reduction of $R^{\prime}$ moves even further away from the maximal 0.5 value, and the noise is reduced for small $\theta_{\mathrm{SO}}$. Around $\theta_{\mathrm{SO}}=\pi$ it increases from zero, because of the additional partition noise. For the triplet, the maximal value is reached at $\theta=\pi$, where electrons reach the beam splitter with opposite spins, and hence behave classically. The value $S_{33}=0.5 e^{2} / h \nu$ at $\theta_{\mathrm{SO}}=\pi$ for $A=0$ is modified in the presence of backscattering; it is then increased when $R>1 /(2-A)$.

For the cross correlations of the noise between leads 3 and 4 , we find

$$
\begin{aligned}
& S_{34}^{S}(\theta)=-\frac{e^{2}}{h \nu}\left[T R+T^{\prime} R^{\prime}+2 T R^{\prime} \cos (\theta) \delta_{\epsilon_{1}, \epsilon_{2}}\right], \\
& S_{34}^{T_{e_{z}}}=-\frac{e^{2}}{h \nu}\left[T R+T^{\prime} R^{\prime}-2 T R^{\prime} \delta_{\epsilon_{1}, \epsilon_{2}}\right], \\
& S_{34}^{T_{u_{\uparrow}, z}}(\theta)=-\frac{e^{2}}{h \nu}\left[T R+T^{\prime} R^{\prime}-2 T R^{\prime} \cos ^{2}(\theta / 2) \delta_{\epsilon_{1}, \epsilon_{2}}\right],
\end{aligned}
$$

with $T^{\prime}=T(1-A)$. These expressions are very similar to the ones for the autocorrelations (103)-(105). The main difference is the negative sign of these correlations, which comes from the fact that $S_{33}+S_{34}=0$ when $A=0$. The other difference is that the term $R^{\prime}\left(1-R^{\prime}\right)$ is replaced by $R^{\prime} T^{\prime}$, which is easily understandable since the cross-correlation involves $\left\langle n_{3}\right\rangle\left\langle n_{4}\right\rangle=R^{\prime} T^{\prime}$ for a particle injected in lead 1, while the autocorrelation involves $\left\langle n_{3}\right\rangle\left(1-\left\langle n_{3}\right\rangle\right)=R^{\prime}\left(1-R^{\prime}\right)$, see Appendix C.

\section{Spin-polarized case}

We now turn to the case of injection from a spin-polarized Fermi liquid lead with a continuous energy spectrum. We start from the standard expression for the noise, ${ }^{59}$ which corresponds to the continuous version of Eqs. (44)-(46)

$$
\begin{aligned}
S_{\alpha \beta}= & \frac{e^{2}}{h} \operatorname{Re} \int d \epsilon \sum_{\gamma \delta u v} A_{\gamma \delta}^{u v}(\alpha, \epsilon, \epsilon) A_{\delta \gamma}^{v u}(\beta, \epsilon, \epsilon) \\
& \times f_{\gamma u}(\epsilon)\left[1-f_{\delta v}(\epsilon)\right],
\end{aligned}
$$

where $f_{\alpha u}(\epsilon)=\left\langle a_{\alpha u}^{\dagger} a_{\alpha u}\right\rangle$ and $u=(\sigma, n)$. Here, again, $\sigma=\uparrow, \downarrow$ denotes the spin components and $n=a, b$ the band index. At zero temperature, for the scattering matrix of the beamsplitter with a two-band spin-orbit active region in one of its incoming arms (lead 1), Eqs. (48) and (49), we obtain for the shot noise in the outgoing leads (say, lead 3),

$$
\begin{aligned}
S_{33}= & \frac{e^{2}}{h} \int d \epsilon T(1-T) \sum_{\sigma \sigma^{\prime} n m}\left|U_{\sigma n, \sigma^{\prime} m}^{c c}\right|^{2}\left\{f_{1 \sigma n}(\varepsilon)\left[1-f_{2 \sigma^{\prime} m}(\varepsilon)\right]\right. \\
& \left.+f_{2 \sigma n}(\varepsilon)\left[1-f_{1 \sigma^{\prime} m}(\varepsilon)\right]\right\},
\end{aligned}
$$

where $U_{\sigma n, \sigma^{\prime} m}^{c c}$ denote the matrix elements of $\mathbf{U}_{\text {SO }}^{c c}$ given in Eq. (42).

\section{Injection into one subband}

We consider a (nonequilibrium) spin-polarized injection into leads 1 and 2 in subband $a$ only, which we model with

$$
\begin{gathered}
\mu_{1 \uparrow a}=\mu_{2 \uparrow a}=\varepsilon_{F}+e V, \\
\mu_{1 \downarrow a}=\mu_{2 \downarrow a}=\varepsilon_{F}+\frac{1-p}{1+p} e V, \\
\mu_{1 \sigma b}=\mu_{2 \sigma b}=\varepsilon_{F} \quad(\sigma=\uparrow, \downarrow), \\
\mu_{3 \sigma n}=\mu_{4 \sigma n}=\varepsilon_{F} \quad(\sigma=\uparrow, \downarrow ; n=a, b) .
\end{gathered}
$$

The degree of spin polarization is controlled by the parameter $0 \leqslant p \leqslant 1$. For full polarization $p=1$ there is no voltage drop for spin-down electrons in Eq. (113), which therefore do not contribute to transport. Note that here we only inject electrons in channel $a$, see Eqs. (114) and (115). We then obtain for the current in the outgoing leads

$$
I_{3}=I_{4}=\frac{e^{2}}{h} V \frac{2}{1+p},
$$

which gets halved for full polarization, $p=1$, as compared to the unpolarized case $p=0$. For the autocorrelation noise we find

$$
\begin{aligned}
S_{33}= & \frac{2 e^{2}}{h} T(1-T)\left[\frac{2 p}{1+p}\left|U_{\uparrow a ; \downarrow a}^{c c}\right|^{2}+\left|U_{\uparrow a ; \downarrow b}^{c c}\right|^{2}+\left|U_{\uparrow a ; \uparrow b}^{c c}\right|^{2}\right. \\
& \left.+\frac{1-p}{1+p}\left(\left|U_{\downarrow a ; \uparrow b}^{c c}\right|^{2}+\left|U_{\downarrow a ; \downarrow b}^{c c}\right|^{2}\right)\right] e V .
\end{aligned}
$$

Note that $S_{33}=S_{44}=-S_{34}$. We define the reduced Fano factor $f_{p} \equiv F / T(1-T) \equiv S_{33} / 2 e I_{3} T(1-T)$, and using Eq. (42) and $|\xi|^{2}=1$ we find

$$
f_{p}=\frac{p}{4}\left(\cos ^{2} \frac{\theta_{d}}{2}+1-2 \cos \frac{\theta_{d}}{2} \cos \theta_{\mathrm{SO}}\right)+\frac{1}{2} \sin ^{2} \frac{\theta_{d}}{2} .
$$

This agrees with Ref. 69 for $\beta=0$ and $p=1$. Notice, however, that if $p<1$ there is an additional correction, i.e., the last term in Eq. (118), which is independent of $p$ and thus there is a small noise power even for the unpolarized case $p=0$. This is related to the presence of the additional empty channel $b$, which allows for fluctations in the outgoing leads in the case of finite SO-induced interband coupling, $\theta_{d} \neq 0$. In the 
strictly 1D case (i.e., single channel), this effect disappears as $\theta_{d}=0$ and Eq. (118) reduces to the expression

$$
f_{p}=\frac{p}{2}\left(1-\cos \theta_{\mathrm{SO}}\right)=p \sin ^{2} \frac{\theta_{\mathrm{SO}}}{2} .
$$

This effect also vanishes in the case where both bands are occupied, studied in the next section.

\section{Injection into both leads}

We now consider the more realistic case of injection into both subbands $a$ and $b$ and model it with

$$
\begin{gathered}
\mu_{1 \uparrow}=\mu_{2 \uparrow}=\varepsilon_{F}+e V, \\
\mu_{1 \downarrow}=\mu_{2 \downarrow}=\varepsilon_{F}+\frac{1-p}{1+p} e V, \\
\mu_{3 \sigma}=\mu_{4 \sigma}=\varepsilon_{F} \quad(\sigma=\uparrow, \downarrow),
\end{gathered}
$$

where $\mu_{i, \sigma, a}=\mu_{i, \sigma, b}=\mu_{i, \sigma}$. We obtain the current

$$
I_{3}=I_{4}=\frac{2 e^{2}}{h} V \frac{2}{1+p},
$$

which is twice the current in the case of injection into a single channel given by Eq. (116). The Fano factor reads

$$
F=T(1-T) \frac{p}{2} \sum_{n, m=a, b}\left|U_{\uparrow n, \downarrow m}^{c c}\right|^{2} .
$$

Using Eq. (42), we obtain the reduced Fano factor $f_{p}$ $\equiv F / T(1-T)$,

$$
f_{p}=\frac{p}{2}\left(1-\cos \frac{\theta_{d}}{2} \cos \theta_{\mathrm{SO}}\right) .
$$

This vanishes in the case of unpolarized injection $p=0$, showing that the interband coupling in lead 1 in itself does not give rise to additional noise for two filled channels. For an uncoupled channel $\left(\theta_{d}=0\right), f_{p}$ yields the usual form valid for single channels, i.e., Eq. (119). The above results were derived for a quantization axis along the $z$ direction. For other directions, one can derive similar but distinct formulas. Finally, we note that measuring such Fano factors enables one to quantify the degree of spin polarization $p$ of the reservoirs along different quantization axis.

\section{E. Coherent injection into multiple discrete states}

Here we generalize our previous results by studying the injection of electrons into leads with a discrete spectrum. We show that even in the case of injection into many different discrete states of the lead, we can observe two-particle coherence, e.g., bunching and antibunching, and thus detect entangled states. We will identify six asymptotic regimes separated by the relative magnitude of the level spacing $\delta$ in the leads, the energy mismatch $\Delta$ of the injected electrons, and the energy broadening $\gamma$ of the injected electrons. It turns out that in four of the six regimes, we obtain (asymptotically) full two-particle interference. Only in two regimes,

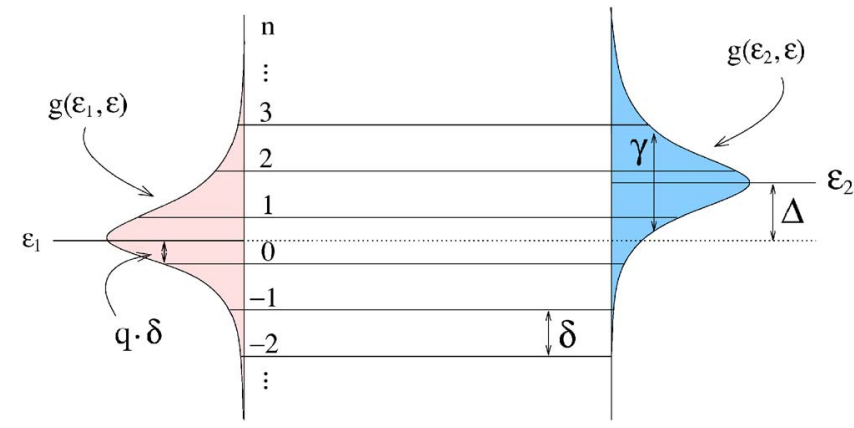

FIG. 6. (Color online) Injection of electrons into equidistant discrete lead states $n=0, \pm 1, \pm 2, \ldots$, with spacing $\delta$ at center energies $\varepsilon_{1,2}$ separated by $\Delta=\varepsilon_{2}-\varepsilon_{1}$ with distributions $g\left(\varepsilon_{1,2}, \varepsilon\right)$ of width $\gamma$. The distributions $g\left(\varepsilon_{1,2}, \varepsilon\right)$ are not drawn to scale, as their normalizations depend on $q$.

namely when the energy mismatch $\Delta$ exceeds both $\delta$ and $\gamma$, we obtain no two-particle interference.

Electron injection into leads 1 and 2 is assumed to be coherent but with a finite width $\gamma$ in energy, such that several energy levels of the lead can be filled. This represents a generalization of both the injection into single discrete levels as discussed in this paper and in earlier works ${ }^{6,28}$ and of recent investigations of the problem in the continuum limit ${ }^{61}$ which will be a special case of the following discussion.

We assume here injection into leads with equidistant levels $\varepsilon_{n}=n \delta+q \delta$, where $n=0, \pm 1, \pm 2, \ldots$ and $0 \leqslant q<1$ is a fixed fractional offset (see Fig. 6). The assumption of equidistance simplifies our calculations because it allows us to perform the discrete sums explicitly (Appendix E). Also, we choose our discrete index $n$ to run from $-\infty$ to $+\infty$, this is done for convenience and merely means that the spectrum is well described within an energy band a few times $\gamma$ wide. We do not expect our results and conclusions to be significantly altered in cases of nonequidistant energy levels in the leads.

Injection of an electron with spin $\sigma=\uparrow, \downarrow$ into lead $\alpha$ and centered about the energy $\varepsilon$ is described by the creation operator

$$
c_{\alpha \sigma}^{\dagger}(\varepsilon)=\sum_{n=-\infty}^{\infty} g\left(\varepsilon, \varepsilon_{n}\right) a_{\alpha \sigma}^{\dagger}\left(\varepsilon_{n}\right),
$$

where $a_{\alpha \sigma}^{\dagger}\left(\varepsilon_{n}\right)$ creates an electron with the sharp energy $\varepsilon_{n}$, see Eq. (44) and below. The weight function $g$ will be assumed to have the Breit-Wigner form

$$
g\left(\varepsilon, \varepsilon^{\prime}\right)=\frac{g_{0}(\varepsilon)}{\varepsilon-\varepsilon^{\prime}+i \gamma},
$$

with the normalization condition $\sum_{n=-\infty}^{\infty}\left|g\left(\varepsilon, \varepsilon^{\prime}\right)\right|^{2}=1$. Unlike for the case of weak tunneling from, e.g., a quantum $\operatorname{dot}^{8}{ }^{8}$ we assume here that the single-electron states are filled with probability 1 , but with uncertain energy. This can be achieved with time-dependent tunnel barriers. Note that for widely spaced levels $\delta \gg \gamma$ and away from symmetric injection into two adjacent levels $(q=1 / 2)$, injection thus takes place into the nearest level with probability $\approx 1$. The two- 
particle injected states we are interested in are

$$
\left.\begin{array}{c}
|S\rangle \\
\left|T_{e_{i}}\right\rangle
\end{array}\right\}=\frac{1}{\sqrt{2}}\left[c_{1 \uparrow}^{\dagger}\left(\varepsilon_{1}\right) c_{2 \downarrow}^{\dagger}\left(\varepsilon_{2}\right) \mp c_{1 \downarrow}^{\dagger}\left(\varepsilon_{1}\right) c_{2 \uparrow}^{\dagger}\left(\varepsilon_{2}\right)\right],
$$

the singlet and entangled triplet states with single-particle energies centered around $\varepsilon_{1}$ and $\varepsilon_{2}$ and smeared over a width $\gamma$. Using Eq. (126), these states can be expressed in terms of the states with sharp energies (50) as $\left|S, T_{e_{i}}\right\rangle$ $=\Sigma_{\varepsilon_{1}^{\prime}, \varepsilon_{2}^{\prime}} g\left(\varepsilon_{1}, \varepsilon_{1}^{\prime}\right) g\left(\varepsilon_{2}, \varepsilon_{2}^{\prime}\right)\left|S, T_{e_{i}}\right\rangle_{\varepsilon_{1}^{\prime}, \varepsilon_{2}^{\prime}}$. Using the normalization condition, we find that the average current in the outgoing leads of the beamsplitter is unaffected by the spread in energy, i.e., $I_{3}=I_{4}=-e / h \nu$. For the Fano factor $F=S_{33} / 2 e I_{3}$, we find

$$
F_{S, T_{e}}=T(1-T)\left[1 \pm\left|h\left(\varepsilon_{1}, \varepsilon_{2}\right)\right|^{2}\right] .
$$

The discrete overlap function $h$ is given by

$$
h\left(\varepsilon_{1}, \varepsilon_{2}\right)=\sum_{\varepsilon} g\left(\varepsilon_{2}, \varepsilon\right)^{*} g\left(\varepsilon_{1}, \varepsilon\right) .
$$

The discrete summations required to evaluate the function $h$ are carried out in Appendix $\mathrm{E}$ and lead to the main result of this section $\left(\Delta \equiv \varepsilon_{2}-\varepsilon_{1}\right)$,

$$
|h|^{2}=\frac{\gamma^{2}}{(\Delta / 2)^{2}+\gamma^{2}} \frac{\cosh ^{2}(2 \pi \gamma / \delta)-\cos ^{2}(\pi \Delta / \delta)}{\sinh ^{2}(2 \pi \gamma / \delta)} .
$$

Note that $|h|^{2}$ appears in Eq. (129) for the Fano factor and generalizes the Kronecker delta for the case of injection into a single level. The function $0<|h(\Delta, \delta, \gamma)|^{2} \leqslant 1$ determines the amount of two-particle interference that can be observed (see Fig. 7). Note that as a consequence of the normalization of $g\left(\varepsilon, \varepsilon_{n}\right)$, the overlap function $|h|^{2}$ is independent of the energy offset $q$ (see Appendix E). For $|h|^{2}=1$, full bunching and antibunching of singlets and entangled triplets can be expected which is ideal for the purpose of discriminating these entangled states from unentangled two-particle states. If $|h|^{2} \rightarrow 0$, then no interference, i.e., no bunching or antibunching will be observed because the two wavepackets centered around $\varepsilon_{1}$ and $\varepsilon_{2}$ have no overlap in energy space.

Since $|h(\Delta, \delta, \gamma)|^{2}$ depends on three parameters with the dimension of an energy, there are $3 !=6$ different asymptotic regimes. We will now discuss all six cases, grouped into three sections with $\Delta, \gamma$, and $\delta$ as the smallest energy, respectively. We also note that since $|h|^{2}$ is a dimensionless number, it is in fact only a function of two dimensionless energy ratios, e.g., $\Delta / \delta$ and $\delta / \gamma$, (see Fig. 7) as can easily be seen from Eq. (131).

\section{Matching energies $\varepsilon_{1}=\varepsilon_{2}(\Delta \ll \gamma, \delta)$}

If $\Delta=\varepsilon_{1}-\varepsilon_{2}=0$, then $|h|^{2}=1$ for arbitrary values of the other three parameters $\delta, \gamma$, and $q$. In other words, if the two electrons are injected with energy distributions whose centers coincide, then the interference is always maximal. This result persists for finite $\Delta$ as long as $\Delta \ll \gamma, \delta$; in this case, the correction is $O\left(\Delta^{2}\right)$. For $\Delta \ll \gamma \ll \delta$, we find $|h|^{2} \simeq 1$ $-\pi^{2} \Delta^{2} / 3 \delta^{2}$. In the case $\Delta \ll \delta \ll \gamma$, the correction is $|h|^{2} \simeq 1$ $-\Delta^{2} / 4 \gamma^{2}$.

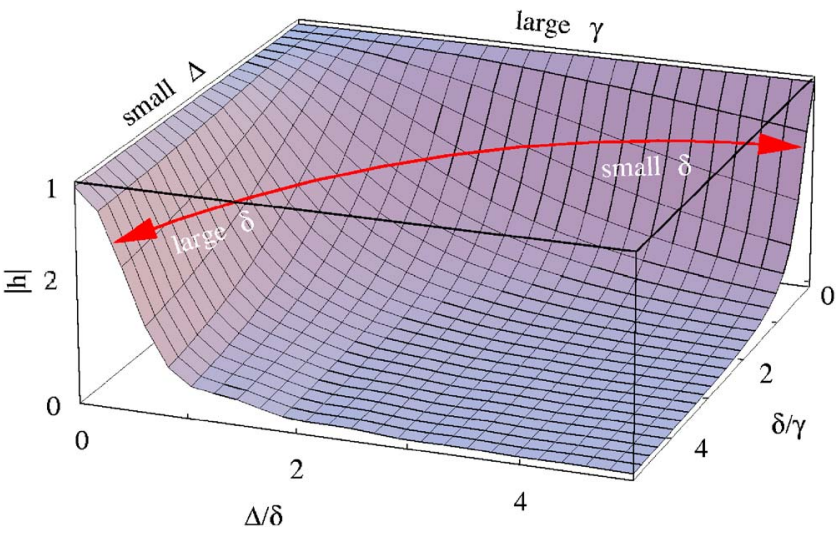

FIG. 7. (Color online) The function $|h(\Delta, \delta, \gamma)|^{2}$ as expressed in Eq. (131), plotted versus the dimensionless quantities $\Delta / \delta$ and $\delta / \gamma$, where $\Delta=\varepsilon_{2}-\varepsilon_{1}$ denotes the mean energy difference between the two injected electrons, $\gamma$ the width of their energy distributions, and $\delta$ the level spacing in the leads. The case of matching energies $\varepsilon_{1}$ $\approx \varepsilon_{2}$ or $\Delta \ll \delta, \gamma($ Sec. III E 1$)$ corresponds to the edge indicated as "small $\Delta$ "; here we find $|h|^{2}=1$, irrespective of the ratio $\gamma / \delta$. The case of a broad energy distribution (fast injection) $\gamma \gg \Delta, \delta$ (Sec. III E 4) corresponds to the edge indicated as "large $\gamma$ "; along this edge $|h|^{2}=1$, regardless of $\Delta / \delta$. The red arrow follows a line of constant $\gamma$ and $\Delta$ with variable $\delta$. For large $\delta$, we find again $|h|^{2}$ $=1$ for all $\Delta / \gamma$, whereas for small $\delta$ (continuum limit, Sec. III E 3), the limit $|h|^{2}=0$ or $|h|^{2}=1$ is reached, depending on whether $\Delta / \gamma$ $\gg 1$ or $\Delta / \gamma \ll 1$.

\section{Sharp energies $\gamma \ll \delta, \Delta$}

In the limit of sharp energies, $\gamma \ll \delta, \Delta$, we obtain

$$
|h|^{2}=\frac{\delta^{2}}{\pi^{2} \Delta^{2}} \sin ^{2} \pi \frac{\Delta}{\delta}+O\left(\gamma^{2}\right)
$$

from Eq. (131). The limit $\gamma \ll \delta \ll \Delta$ describes the situation where the two electrons are injected into two different discrete states and thus $|h|^{2} \rightarrow 0$. In the other limit, $\gamma \ll \Delta \ll \delta$, the two electrons are injected into the same discrete level and $|h|^{2}=1-\pi^{2} \Delta^{2} / 3 \delta^{2}+O\left(\Delta^{4} / \delta^{4}, \gamma^{2}\right)$. The limit $\gamma \ll \delta, \Delta$ discussed here describes injection into single discrete levels discussed throughout this paper and in earlier works, ${ }^{6,28,67}$ where $\varepsilon_{1,2}$ are multiples of $\delta$ and $|h|^{2} \rightarrow \delta_{\varepsilon_{1}, \varepsilon_{2}}$ is given by a Kronecker delta.

\section{Continuum limit $\delta \ll \Delta$, $\gamma$}

In the continuum limit, i.e., the asymptotic case where the single-electron level spacing in the leads becomes small compared to all other relevant energy scales, we find a Lorentzian $^{61}$

$$
|h|^{2} \simeq \frac{\gamma^{2}}{(\Delta / 2)^{2}+\gamma^{2}},
$$

with the usual special limits $|h|^{2} \rightarrow 0$ for $\delta \ll \gamma \ll \Delta$ and $|h|^{2}$ $\rightarrow 1$ for $\delta \ll \Delta \ll \gamma$.

\section{Discussion in terms of largest energy scale}

Above, we have discussed all six asymptotic cases, grouped according to the smallest energy scale. Alterna- 
tively, we can discuss the asymptotic regimes characterized by the largest energy scale. In the detuned case $\Delta \gg \delta, \gamma$, we never see two-particle interference since $|h|^{2}$ $\simeq\left(4 \gamma^{2} / \Delta^{2}\right) f(\Delta ; \delta, \gamma) \rightarrow 0$ where $f(\Delta ; \delta, \gamma)$ is a function which is bounded for fixed $\delta$ and $\gamma$. In the case of a wide distribution, such as, e.g., effected by a fast injection into the lead with injection time $\hbar / \gamma$, we are in the regime $\gamma \gg \delta, \Delta$, and we always find that $|h|^{2}$ exponentially approaches 1 . In the single-level case $\delta \gg \gamma, \Delta$, there is only one level to fill and we obtain $|h|^{2} \rightarrow 1$ irrespective of the relative magnitude of $\Delta$ and $\gamma$.

\section{SUMMARY}

We have carried out a thorough study of current and noise for spin-polarized and spin-entangled electrons in a beamsplitter geometry, including a local spin-orbit interaction (Rashba and Dresselhaus) in one of the incoming arms. We have considered incoming leads with one or two channels, as well as backscattering effects. The channels can be coupled via the SO interaction for incoming energies near the band crossing. We have found that the spin-orbit interaction is a useful mechanism to coherently rotate spin states. Such rotation can be used to modulate noise signals, thus providing unique signatures of spin polarization and spin entanglement.

For spin-polarized electrons, noise measurements can give a direct measure of the degree of polarization along different directions. For electron pairs, the coupling between the channels can play an important role. For pairs with incoming energies near the band crossing injected into one of the channels, we find an additional modulation due to the coherent transfer of electrons between the two channels. In this case, noise measurement allows us to distinguish all the different triplets states defined along the $y$ direction, in addition to the singlet. Furthermore, for equal strengths the combined effect of the Rashba and Dresselhaus interactions can partially cancel out. In this case, the spin and orbital degrees of freedom are separable, the interband coupling essentially disappears, and the propagation of spin states is robust against scattering off nonmagnetic impurities.

We have also considered the influence of backscattering in the beam-splitter with a single channel. The main effect is an additional contribution related to the partition noise due to the tunnel barrier describing the backscattering. This reduces the visibility of the oscillations in the shot noise as the SO rotation angle $\theta_{\mathrm{SO}}$ is varied. It also reduces the maximal noise value found for perfect antibunching of singlets.

We have generalized earlier results for the shot noise of entangled electrons by allowing the injection of wave packets, i.e., coherent superpositions of discrete momentum eigenstates (plane waves). We have found a general analytical formula for the two-particle interference visibility $|h|^{2}$ in terms of all three relevant energy scales $\Delta, \gamma$, and $\delta$. Our new result contains and generalizes both the discrete single-level case and the continuum case.

Finally, we have developed a simple heuristic picture for the noise based on number operators in the different leads and the relevant transmission and reflection probability amplitudes in the beamsplitter. Within this picture we can more intuitively rederive some of the formulas for the noisepreviously derived within the rigorous scattering formalism (Sec. III) - in the presence of spin-orbit interaction and backscattering in the incoming leads.

\section{ACKNOWLEDGMENTS}

The authors acknowledge useful discussions with S. Erlingsson. This work was supported by NCCR Nanoscale Science, CNPq, FAPESP, EU-Spintronics, Swiss NSF, DARPA, $\mathrm{ARO}$, and ONR.

\section{APPENDIX A: BOUNDARY CONDITIONS AT $x=L$}

Here we show in some detail that both the wave function and the velocity operator acting on it are continuous at the exit $(x=L)$ of the SO region in lead 1.

\section{Continuity of $\Psi(x, y)$}

The continuity of the wave function at $x=L$ is trivially satisfied by the two-channel state

$$
\begin{aligned}
\Psi(x, y)= & \left(\begin{array}{c}
\cos \left(\theta_{d} / 2\right) e^{-i \theta_{\mathrm{SO}} / 2}+e^{i \theta_{\mathrm{SO}} / 2} \\
\xi\left[\cos \left(\theta_{d} / 2\right) e^{-i \theta_{\mathrm{SO}} / 2}-e^{i \theta_{\mathrm{SO}} / 2}\right]
\end{array}\right) \frac{1}{2} e^{i\left(k_{c}+k_{\mathrm{SO}}\right) x} \phi_{a}(y) \\
& +\left(\begin{array}{c}
-i \sin \left(\theta_{d} / 2\right) e^{-i \theta_{\mathrm{SO}} / 2} \\
i \xi \sin \left(\theta_{d} / 2\right) e^{-i \theta_{\mathrm{SO}} / 2}
\end{array}\right) \frac{1}{2} e^{i\left(k_{c}-k_{\mathrm{SO}}\right) x} \phi_{b}(y),
\end{aligned}
$$

describing the electron state within the SO region $0 \leqslant x \leqslant L$ and the state

$$
\Phi(x, y)=\left(\begin{array}{l}
A \\
B
\end{array}\right) \frac{1}{2} e^{i\left(k_{c}+k_{\mathrm{SO}}\right) x} \phi_{a}(y)+\left(\begin{array}{l}
C \\
D
\end{array}\right) \frac{1}{2} e^{i\left(k_{c}-k_{\mathrm{SO}}\right) x} \phi_{b}(y),
$$

valid for $x \geqslant L$ in lead 1 , if we choose $A, B, C$, and $D$, equal to the corresponding components of $\Psi(L, y)$.

\section{Continuity of the current flow}

The continuity of the (nondiagonal) velocity operator ${ }^{55}$

$$
\hat{v}_{\mathrm{SO}}=\left(\begin{array}{cccc}
\frac{\hbar}{i m} \partial_{x} & \frac{\beta+i \alpha}{\hbar} & 0 & 0 \\
\frac{\beta-i \alpha}{\hbar} & \frac{\hbar}{i m} \partial_{x} & 0 & 0 \\
0 & 0 & \frac{\hbar}{i m} \partial_{x} & \frac{\beta+i \alpha}{\hbar} \\
0 & 0 & \frac{\beta-i \alpha}{\hbar} & \frac{\hbar}{i m} \partial_{x}
\end{array}\right)
$$

acting on the wave fuctions at $x=L$

$$
\left.\hat{v}_{\mathrm{SO}} \Psi(x, y)\right|_{x \rightarrow L^{-}}=\left.\hat{v}_{\mathrm{SO}} \Phi(x, y)\right|_{x \rightarrow L^{+}},
$$

assures current conservation. The left-hand side of Eq. (A4) yields 


$$
\left.\hat{v}_{\mathrm{SO}} \Psi(x, y)\right|_{x \rightarrow L^{-}}=\frac{\hbar}{4 m}\left[\left(\begin{array}{c}
\left(k_{c}-\Delta / 2+k_{\mathrm{SO}}\right) e^{-i \Delta L / 2} \\
\xi\left(k_{c}-\Delta / 2+k_{\mathrm{SO}}\right) e^{-i \Delta L / 2} \\
\left(k_{c}-\Delta / 2-k_{\mathrm{SO}}\right) e^{-i \Delta L / 2} \\
-\xi\left(k_{c}-\Delta / 2-k_{\mathrm{SO}}\right) e^{-i \Delta L / 2}
\end{array}\right)+\left(\begin{array}{c}
\left(k_{c}+\Delta / 2+k_{\mathrm{SO}}\right) e^{i \Delta L / 2} \\
\xi\left(k_{c}+\Delta / 2+k_{\mathrm{SO}}\right) e^{i \Delta L / 2} \\
-\left(k_{c}+\Delta / 2-k_{\mathrm{SO}}\right) e^{i \Delta L / 2} \\
\xi\left(k_{c}+\Delta / 2-k_{\mathrm{SO}}\right) e^{i \Delta L / 2}
\end{array}\right)\right] e^{i k_{c} L}+\frac{\hbar}{2 m}\left(\begin{array}{c}
\left(k_{2}-k_{\mathrm{SO}}\right) e^{i k_{2} L} \\
-\xi\left(k_{2}-k_{\mathrm{SO}}\right) e^{i k_{2} L} \\
0 \\
0
\end{array}\right)
$$

or

$$
\begin{aligned}
\left.\hat{v}_{\mathrm{SO}} \Psi(x, y)\right|_{x \rightarrow L^{-}}= & \frac{\hbar}{2 m}\left(\begin{array}{c}
\left(k_{c}+k_{\mathrm{SO}}\right)\left[\left(\cos \left(\theta_{d} / 2\right)+i \frac{\Delta}{\left(k_{c}+k_{\mathrm{SO}}\right)} \sin \left(\theta_{d} / 2\right)\right) e^{-i \theta_{\mathrm{SO}} / 2}+e^{i \theta_{\mathrm{SO}} / 2}\right] \\
\xi\left(k_{c}+k_{\mathrm{SO}}\right)\left[\left(\cos \left(\theta_{d} / 2\right)+i \frac{\Delta}{\left(k_{c}+k_{\mathrm{SO}}\right)} \sin \left(\theta_{d} / 2\right)\right) e^{-i \theta_{\mathrm{SO}} / 2}-e^{i \theta_{\mathrm{SO}} / 2}\right]
\end{array}\right) e^{i\left(k_{c}+k_{\mathrm{SO}}\right) L} \phi_{a}(y) \\
& +\frac{\hbar}{2 m}\left(\begin{array}{c}
-i\left(k_{c}-k_{\mathrm{SO}}\right)\left(\sin \left(\theta_{d} / 2\right)+i \frac{\Delta}{\left(k_{c}-k_{\mathrm{SO}}\right)} \cos \left(\theta_{d} / 2\right)\right) e^{i \theta_{\mathrm{SO}} / 2} \\
-i \xi\left(k_{c}-k_{\mathrm{SO}}\right)\left(\sin \left(\theta_{d} / 2\right)+i \frac{\Delta}{\xi\left(k_{c}-k_{\mathrm{SO}}\right)} \cos \left(\theta_{d} / 2\right)\right) e^{i \theta_{\mathrm{SO}} / 2}
\end{array}\right) e^{i\left(k_{c}-k_{\mathrm{SO}}\right) L} \phi_{b}(y),
\end{aligned}
$$

where we used $k_{2}-k_{\mathrm{SO}}=k_{c}+k_{\mathrm{SO}}$. On the other hand, it is straightforward to show that

$$
\begin{aligned}
\left.\hat{v}_{\mathrm{SO}} \Phi(x, y)\right|_{x \rightarrow L^{+}} & \frac{\hbar}{2 m}\left(k_{c}+k_{\mathrm{SO}}\right)\left(\begin{array}{l}
{\left[\cos \left(\theta_{d} / 2\right) e^{-i \theta_{\mathrm{SO}} / 2}+e^{i \theta_{\mathrm{SO}} / 2}\right]} \\
\xi\left[\cos \left(\theta_{d} / 2\right) e^{-i \theta_{\mathrm{SO}} / 2}-e^{i \theta_{\mathrm{SO}} / 2}\right]
\end{array}\right) \\
& \times e^{i\left(k_{c}+k_{\mathrm{SO}}\right) L} \phi_{a}(y)+\frac{\hbar}{2 m}\left(k_{c}-k_{\mathrm{SO}}\right) \\
& \times\left(\begin{array}{c}
-i \sin \left(\theta_{d} / 2\right) e^{i \theta_{\mathrm{SO}} / 2} \\
-i \xi \sin \left(\theta_{d} / 2\right) e^{i \theta_{\mathrm{SO}} / 2}
\end{array}\right) \\
& \times e^{i\left(k_{c}-k_{\mathrm{SO}}\right) L} \phi_{b}(y) .
\end{aligned}
$$

Again, assuming $\Delta \ll k_{c} \sim k_{F}$ we can drop the terms proportional to $\Delta /\left(k_{c} \pm k_{\mathrm{SO}}\right)$ in Eq. (A6) thus arriving at the desired equality $\left.\hat{v}_{\mathrm{SO}} \Psi(x, y)\right|_{x \rightarrow L^{-}}=\left.\hat{v}_{\mathrm{SO}} \Phi(x, y)\right|_{x \rightarrow L^{+}}$which assures current conservation.

\section{APPENDIX B: TRANSPORT THROUGH QUANTUM WIRE AT $|\boldsymbol{\alpha}|=\boldsymbol{\beta}$}

As an example of a backscattering mechanism in quasi-1D channels, we calculate here the transmission and reflection coefficients for a quantum wire of length $a$ in the presence of spin-orbit coupling of both the Rashba and Dresselhaus types with equal strengths $|\alpha|=\beta$. The wire is attached to leads which are modeled as semi-infinite quantum wires without spin-orbit interaction. Again we choose the axis of the wire along the $x$ direction. The central region $0 \leqslant x \leqslant a$ is characterized by an effective mass $m_{2}$ and a confining potential $V_{2}(y)$, whereas the leads $(x<0$ and $x>a)$ have effective mass $m_{1}$ and confining potential $V_{1}(y)$, in general $m_{1} \neq m_{2}, V_{1} \neq V_{2}$. For definiteness let us consider the case $\alpha=-\beta$. Here the spin is independent of the momentum along the wire thus being a good quantum number. The problem of matching the wave functions at the interfaces separates then into these two spin directions. Without loss of generality, we will concentrate on the + state as defined in Eq. (2). Moreover, to analyze ballistic transport through this arrangement we will use the approximation that the reflected and transmitted electrons have the same subband index as the incoming ones. Thus, the incoming and reflected parts of the wave function at $x<0$ read

$$
\psi(x, y)=\left(\lambda e^{i k x}+A e^{-i k x}\right) \phi_{n}^{1}(y),
$$

and for the transmitted part at $x>a$ we have

$$
\psi(x, y)=C e^{i k x} \phi_{n}^{1}(y) .
$$

Here $k$ is the wave vector of the incoming particle along the wire axis with energy $\varepsilon=\varepsilon_{n}^{1}+(\hbar k)^{2} / 2 m_{1}$, where $\varepsilon_{n}^{1}$ is the subband energy of the transverse wave function $\varphi_{n}^{1}(y)$ according to the potential $V_{1}(y)$. In the above equations, $\lambda$ is the amplitude of the incoming wave, and $A$ and $C$ are the amplitudes of the reflected and transmitted wave, respectively. Note that the above form of the transmitted and reflected part of the wave function are a restricted ansatz containing the aforementioned approximation that the subband index is the same as in the incoming part. For the wave function in the region with spin-orbit coupling $(0 \leqslant x \leqslant a)$ we use the general ansatz

$$
\psi(x, y)=\sum_{j}\left[\left(B_{+}^{j} e^{i q_{+}^{j} x}+B_{-}^{j} e^{i q_{-}^{j} x}\right) \phi_{j}^{2}(y) e^{i \sqrt{2} \alpha m_{2} y}\right]
$$

with

$$
q_{ \pm}^{j}=-\sqrt{2} \alpha m_{2} \pm \sqrt{\frac{2 m_{2}}{\hbar^{2}}\left(\varepsilon+\frac{2 m_{2} \alpha^{2}}{\hbar^{2}}-\varepsilon_{j}^{2}\right)},
$$

and $\varepsilon_{j}^{2}$ is the subband energy of the transverse wave function $\varphi_{j}^{2}$ in the absence of spin-orbit coupling. The above ansatz 
contains all subbnads, and the corresponding wave vectors $q_{ \pm}^{j}$ will acquire imaginary parts for large enough subband energies. However, since this region of the setup is finite $(0 \leqslant x \leqslant a)$, the wave functions remain normalizable.

The continuity conditions on the wave functions at the interface $x=0$ lead to

$$
\begin{aligned}
(\lambda+A) \phi_{n}^{1}(y) & =\sum_{j}\left[\left(B_{+}^{j}+B_{-}^{j}\right) \phi_{j}^{2}(y) e^{i \sqrt{2} \alpha m_{2} y}\right], \\
\frac{m_{2}}{m_{1}} k(\lambda-A) \phi_{n}^{1}(y) & =\sum_{j}\left[\left(\eta_{j} B_{+}^{j}-\eta_{j} B_{-}^{j}\right) \phi_{j}^{2}(y) e^{i \sqrt{2} \alpha m_{2} y}\right]
\end{aligned}
$$

with

$$
\eta_{j}=\sqrt{\frac{2 m_{2}}{\hbar^{2}}\left(\varepsilon+\frac{2 m_{2} \alpha^{2}}{\hbar^{2}}-\varepsilon_{j}^{2}\right)},
$$

and at $x=a$ one has

$$
\begin{gathered}
C e^{i k a} \phi_{n}^{1}(y)=\sum_{j}\left[\left(B_{+}^{j} e^{i q_{+}^{j} a}+B_{-}^{j} e^{i q_{-}^{j} a}\right) \phi_{j}^{2}(y) e^{i \sqrt{2} \alpha m_{2} y}\right], \\
\frac{m_{2}}{m_{1}} k C e^{i k a} \phi_{n}^{1}(y)=\sum_{j}\left[\left(\eta_{j} B_{+}^{j} e^{i q_{+}^{j} a}-\eta_{j} B_{-}^{j} e^{i q_{-}^{j} a}\right)\right. \\
\left.\times \phi_{j}^{2}(y) e^{i \sqrt{2} \alpha m_{2} y}\right] .
\end{gathered}
$$

Multiplying the above by $\left[\phi_{j}^{2}(y)\right]^{*} e^{-i \sqrt{2} \alpha m_{2} y}$ and integrating over the transverse direction $y$ gives

$$
\begin{gathered}
(\lambda+A) S_{j n}=B_{+}^{j}+B_{-}^{j}, \\
\frac{m_{2}}{m_{1}} k(\lambda-A) S_{j n}=\eta_{j} B_{+}^{j}-\left[\eta_{j} B_{-}^{j},\right. \\
C e^{i k a} S_{j n}=B_{+}^{j} e^{i q_{+}^{j} a}+B_{-}^{j} e^{i q_{-}^{j} a}, \\
\frac{m_{2}}{m_{1}} k C e^{i k a} S_{j n}=\eta_{j} B_{+}^{j} e^{i q_{+}^{j} a}-\eta_{j} B_{-}^{j} e^{i q_{-}^{j} a},
\end{gathered}
$$

where we have defined the overlap integrals

$$
S_{j n}=\int d y\left[\phi_{j}^{2}(y)\right]^{*} e^{+i \sqrt{2} \alpha m_{2} y} \phi_{n}^{1}(y) .
$$

Eliminating the quantities $A$ and $C$ yields

$$
\begin{aligned}
& 2 \frac{m_{2}}{m_{1}} k \lambda S_{j n}=\left(\frac{m_{2}}{m_{1}} k+\eta_{j}\right) B_{+}^{j}+\left(\frac{m_{2}}{m_{1}} k-\eta_{j}\right) B_{-}^{j}, \\
& 0=\left(\frac{m_{2}}{m_{1}} k-\eta_{j}\right) B_{+}^{j} e^{i q_{+}^{j} a}+\left(\frac{m_{2}}{m_{1}} k+\eta_{j}\right) B_{-}^{j} e^{i q_{-}^{j} a}
\end{aligned}
$$

or, solving for $B_{ \pm}^{j}$,

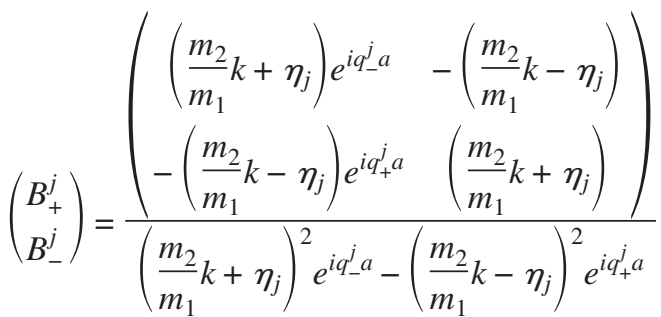

$$
\begin{aligned}
& \times\left(\begin{array}{c}
2 \frac{m_{2}}{m_{1}} k \lambda S_{j n} \\
0
\end{array}\right) \text {. }
\end{aligned}
$$

Moreover, multipying Eqs. (B5)-(B9) by $\left[\phi_{n}^{1}(y)\right]^{*}$ and integrating over $y$ gives

$$
\lambda+A=\sum_{j}\left[\left(B_{+}^{j}+B_{-}^{j}\right) S_{j n}^{*}\right]
$$

$$
\frac{m_{2}}{m_{1}} k(\lambda-A)=\sum_{j}\left[\left(\eta_{j} B_{+}^{j}-\eta_{j} B_{-}^{j}\right) S_{j n}^{*}\right]
$$

$$
C e^{i k a}=\sum_{j}\left[\left(B_{+}^{j} e^{i q_{+}^{j} a}+B_{-}^{j} e^{i q_{-}^{j} a}\right) S_{j n}^{*}\right]
$$

$$
\frac{m_{2}}{m_{1}} k C e^{i k a}=\sum_{j}\left[\left(\eta_{j} B_{+}^{j} e^{i q_{+}^{j} a}-\eta_{j} B_{-}^{j} e^{i q_{-}^{j} a}\right) S_{j n}^{*}\right] .
$$

Inserting the above expressions for $B_{ \pm}^{j}$ into Eq. (B18) yields the following result for the reflection amplitudes

$$
\begin{aligned}
\frac{A}{\lambda}= & -1 \\
& +\sum_{j} \frac{\left[\left(\frac{m_{2}}{m_{1}} k+\eta_{j}\right) e^{-i \eta_{j} a}-\left(\frac{m_{2}}{m_{1}} k-\eta_{j}\right) e^{i \eta_{j} a}\right] 2 \frac{m_{2}}{m_{1}} k\left|S_{j n}\right|^{2}}{\left(\frac{m_{2}}{m_{1}} k+\eta_{j}\right)^{2} e^{-i \eta_{j} a}-\left(\frac{m_{2}}{m_{1}} k-\eta_{j}\right)^{2} e^{i \eta_{j} a}},
\end{aligned}
$$

and from Eq. (B20) one finds for the transmission amplitude

$$
\frac{C}{\lambda}=\sum_{j} \frac{e^{-i k a-i \sqrt{2} \alpha m_{2} a} 4 \eta_{j} \frac{m_{2}}{m_{1}} k\left|S_{j n}\right|^{2}}{\left(\frac{m_{2}}{m_{1}} k+\eta_{j}\right)^{2} e^{-i \eta_{j} a}-\left(\frac{m_{2}}{m_{1}} k-\eta_{j}\right)^{2} e^{i \eta_{j} a}} .
$$

Equations (B22) and (B23) describe the electron reflection and transmission amplitudes, respectively, arising from the potential discontinuities at the entrance and exit of the SO region $(0 \leqslant x \leqslant a)$. It is worthwhile to note that using Eqs. (B19) and (B21) instead of Eqs. (B18) and (B20) leads to an identical expression for the transmission amplitude and to an equivalent result for the reflection amplitude 


$$
\frac{A}{\lambda}=1-\sum_{j} \frac{\left[\left(\frac{m_{2}}{m_{1}} k+\eta_{j}\right) e^{-i \eta_{j} a}+\left(\frac{m_{2}}{m_{1}} k-\eta_{j}\right) e^{i \eta_{j} a}\right] 2 \eta_{j}\left|S_{j n}\right|^{2}}{\left(\frac{m_{2}}{m_{1}} k+\eta_{j}\right)^{2} e^{-i \eta_{j} a}-\left(\frac{m_{2}}{m_{1}} k-\eta_{j}\right)^{2} e^{i \eta_{j} a}}
$$

This is indeed the same as Eq. (B22) as one can see using $\Sigma_{j}\left|S_{j n}\right|^{2}=1$. Thus, Eqs. (B10)-(B13) [from which Eq. (B17) was obtained] are consistent with Eqs. (B18)-(B21). This is a nontrivial finding since our original ansatz for the wave function was a restricted one containing an approximation, and it is not a priori clear that such an ansatz would lead to a consistent system of equations.

\section{APPENDIX C: HEURISTIC PICTURE OF THE NOISE IN A BEAM SPLITTER}

One can obtain a simple physical picture of the expressions for the current noise by considering the fluctuations of the number operator in the outgoing leads when one injects a pair of particles in leads 1 and 2 (i.e., $\left\langle n_{1}\right\rangle=\left\langle n_{2}\right\rangle=1$ ). For instance,

$$
\begin{gathered}
S_{33}\left(\frac{e^{2}}{h \nu}\right)^{-1} \sim\left\langle\Delta n_{3}^{2}\right\rangle=\left\langle n_{3}^{2}\right\rangle-\left\langle n_{3}\right\rangle^{2}, \\
S_{34}\left(\frac{e^{2}}{h \nu}\right)^{-1} \sim\left\langle\Delta n_{3} \Delta n_{4}\right\rangle=\left\langle n_{3} n_{4}\right\rangle-\left\langle n_{3}\right\rangle\left\langle n_{4}\right\rangle .
\end{gathered}
$$

By considering classical, Fermi or Bose particles we can derive formulas which are in direct correspondence with the results for the current fluctations for electron pairs with spin previously obtained in Sec. III using the rigorous scattering formalism. For clarity we discuss separately the different configurations, illustrated in Fig. 4. We first consider a symmetric beam splitter with backscattering and no local SO effect in lead 1; see Fig. 4(b). We then consider the case of backscattering in the SO lead 1 only, shown in Fig. 4(a). Finally, we return to the SO rotation in a beam splitter with no backscattering.

\section{Backscattering at the beam splitter}

In realistic experiments ${ }^{3}$ the beam-splitter is not perfect, and can actually contain a significant amount of backscattering in all input leads (i.e., $s_{11}, s_{22} \neq 0$ ), as well as "crossbackscattering" between the input leads $\left(s_{12} \neq 0\right)$. The most symmetric scattering matrix corresponding to this situation is

$$
\mathbf{s}=\left(\begin{array}{llll}
a & c & r & t \\
c & a & t & r \\
r & t & a & c \\
t & r & c & a
\end{array}\right),
$$

where all backscattering amplitudes are the same $s_{11}=s_{22}$ $=s_{33}=s_{44}=a$ and so are the cross-backscattering amplitudes $s_{12}=s_{34}=c$. Defining $A=|a|^{2}, C=|c|^{2}, R=|r|^{2}$, and $T=|t|^{2}$, the unitarity of $\mathbf{s}$ imposes $A+C+R+T=1$ and $c=-a r / t$, so that $a$ and $c$ are not independent. To have independent $a$ and $c$, one must drop the requirement of symmetry between input and ouput, as is considered in Sec. III C and Appendix C 2 for the case of backscattering in the SO lead only. We now calculate the expectation values of the number operators in lead 3 and 4 , by simply considering the probabilities for the different scattering configurations. Let $P(3), P(3,3)$, and $P(3,4)$ denote the probabilities of finding one and two electrons in lead 3, and one electron in each lead 3 and 4, respectively. We need to determine $\left\langle n_{3}\right\rangle=P(3)+2 P(3,3),\left\langle n_{3}^{2}\right\rangle$ $=P(3)+4 P(3,3)=\left\langle n_{3}\right\rangle+2 P(3,3)$, and $\left\langle n_{3} n_{4}\right\rangle=P(3,4)$.

For classical particles, we have $\left\langle n_{3}\right\rangle_{C}=\left|s_{13}\right|^{2}\left(\left|s_{21}\right|^{2}+\left|s_{22}\right|^{2}\right.$ $\left.+\left|s_{24}\right|^{2}\right)+\left|s_{23}\right|^{2}\left(\left|s_{11}\right|^{2}+\left|s_{12}\right|^{2}+\left|s_{14}\right|^{2}\right)+2\left|s_{13}\right|^{2}\left|s_{23}\right|^{2}=R+T,\left\langle n_{3}^{2}\right\rangle_{C}$ $=R+T+2 R T$, and $\left\langle n_{3} n_{4}\right\rangle_{C}=\left|s_{13}\right|^{2}\left|s_{24}\right|^{2}+\left|s_{14}\right|^{2}\left|s_{23}\right|^{2}=R^{2}+T^{2}$. We find the autocorrelations and cross correlations

$$
\left\langle\Delta n_{3}^{2}\right\rangle_{C}=T(1-T)+R(1-R)=(A+C)(R+T)+2 R T,
$$

$$
\left\langle\Delta n_{3} \Delta n_{4}\right\rangle_{C}=-2 R T .
$$

As we shall see in Appendix C 2 below, these results correspond to the current noise $S_{33}$ and $S_{34}$ for electrons behaving classically (i.e., with different energies and/or with opposite spins). The result (C1) consists of the sum of two terms corresponding to the partition noise for electrons coming from lead 2 and 1, respectively. One can simply add these contributions because the classical particles are independent. In the second equality we can recognize a partition noise term $(A+C)(R+T)$ in addition to the usual "beam-splitter noise" $\sim 2 R T$.

The situation is different for quantum particles obeying Fermi or Bose statistics, in which case one must first add or substract the amplitudes before building the probabilities for indistiguishable events. One can satisfy unitarity by choosing, for convenience, $\operatorname{Re}\left(r^{*} t\right)=\operatorname{Re}\left(a^{*} c\right)=0$, which yields $\left|r^{2} \pm t^{2}\right|=R \mp T$ and $\left|a^{2} \pm c^{2}\right|=A \mp C$. For spinless fermions, one has $P(3,3)=0$ and $\left\langle n_{3}\right\rangle_{F}=\left|s_{13} s_{24}-s_{14} s_{23}\right|^{2}+\mid s_{13} s_{21}$ $-\left.s_{11} s_{23}\right|^{2}+\left|s_{13} s_{22}-s_{12} s_{23}\right|^{2}=R+T, \quad\left\langle n_{3}^{2}\right\rangle_{F}=\left\langle n_{3}\right\rangle_{F}=R+T, \quad$ and $\left\langle n_{3} n_{4}\right\rangle_{F}=\left|s_{13} s_{24}-s_{14} s_{23}\right|^{2}=(R+T)^{2}$. The correlations read

$$
\left\langle\Delta n_{3}^{2}\right\rangle_{F}=T(1-T)+R(1-R)-2 R T=(A+C)(R+T),
$$

$$
\left\langle\Delta n_{3} \Delta n_{4}\right\rangle_{F}=0 .
$$

For the autocorrelation $\left\langle\Delta n_{3}^{2}\right\rangle_{F}$, we see that the zero value found in the absence of backscattering becomes finite, which is a consequence of the partition noise created by the additionnal backscattering channels. One can obtain this result by substracting from the classical result the forbidden case with two electrons in lead 3, $\left\langle\Delta n_{3}^{2}\right\rangle_{F}=\left\langle\Delta n_{3}^{2}\right\rangle_{C}-2 P(3,3)$. The crosscorrelations, on the other hand, remains zero. These results for fermionic particles correspond to electrons with equal energies in a triplet state.

For spinless bosons, we must double $P(3,3)$ thus obtaining $\left\langle n_{3}\right\rangle_{B}=R+T,\left\langle n_{3}^{2}\right\rangle_{B}=R+T+4 R T$, and $\left\langle n_{3} n_{4}\right\rangle_{B}=(R-T)^{2}$. The correlations are 


$$
\left\langle\Delta n_{3}^{2}\right\rangle_{B}=T(1-T)+R(1-R)+2 R T=(A+C)(R+T)+4 R T,
$$

$$
\left\langle\Delta n_{3} \Delta n_{4}\right\rangle_{B}=-4 R T,
$$

and correspond to electrons in a singlet pair with equal energies. We recognize again the sum of a partition-type noise and the beam-splitter noise $4 R T$.

We can establish a connection between the above results and those of Ref. 67 in which the case with no crossbackscattering $s_{12}=0$ was studied. In that work, the backscattering was introduced in the same way as in Sec. III C, namely, by taking the probabilities $R$ and $T$ from the original beam-splitter and adding a tunnel barrier with reflection probability $R_{B}$. Since the cross-backscattering does not play a direct role for the noise in the output leads, one can identify $A+C \rightarrow R_{B}, R \rightarrow R\left(1-R_{B}\right)$, and $T \rightarrow T\left(1-R_{B}\right)$, which establishes the equivalence of Eqs. (C3) and (C4) and Eqs. (5),(6) of Ref. 67.

\section{Backscattering in one lead}

We consider here the case of backscattering in lead 1 only, which was discussed in Sec. III C. The problem is no longer symmetric and the transmission probabilities $T$ and $R$ are not equivalent anymore. See Appendix B where we calculate explicitly the transmission and reflection coefficients for a model quantum wire with spin-orbit interaction of both the Dresselhaus and Rahsba types. We recall that $R$ and $T$ here are the original quantities before adding the backscattering channel, and therefore satisfy $T=1-R$. We calculate the fluctuations of the number operator in lead $3 n_{3}$ by the same procedure as in Appendix C 1.

For classical particles we find $\left\langle n_{3}\right\rangle_{C}=1-A R,\left\langle n_{3}^{2}\right\rangle_{C}=1$ $-A R+2 T R^{\prime}$, which yields

$$
\left\langle\Delta n_{3}^{2}\right\rangle_{C}=T R+R^{\prime}\left(1-R^{\prime}\right) .
$$

This result corresponds to the current noise expressions (103)-(105) with $\epsilon_{1} \neq \epsilon_{2}$. Hence, in this configuration electrons with distinct energies are not affected by the (anti)symmetrization related to their spin state, and behave, effectively, similar to classical particles.

For fermions, one finds $\left\langle n_{3}\right\rangle_{F}=\left\langle n_{3}^{2}\right\rangle_{F}=1-A R$, which gives

$$
\left\langle\Delta n_{3}^{2}\right\rangle_{F}=T R+R^{\prime}\left(1-R^{\prime}\right)-2 T R^{\prime} .
$$

This result corresponds to the case of triplets (104) and (105) with $\epsilon_{1}=\epsilon_{2}$ and $\theta_{\mathrm{SO}}=0$.

For bosons we can proceed likewise to find $\left\langle n_{3}\right\rangle_{B}=1$ $-A R,\left\langle n_{3}^{2}\right\rangle_{B}=1-A R+4 T R^{\prime}$. The result

$$
\left\langle\Delta n_{3}^{2}\right\rangle_{B}=T R+R^{\prime}\left(1-R^{\prime}\right)+2 T R^{\prime},
$$

corresponds to the case of singlets with $\epsilon_{1}=\epsilon_{2}$ and $\theta_{\mathrm{SO}}=0$. This can also be found by doubling the probability to have electrons in the same lead $3,\left\langle\Delta n_{3}^{2}\right\rangle_{B}=\left\langle\Delta n_{3}^{2}\right\rangle_{C}+2 P(3,3)$.

We can also rewrite the results above as

$$
\begin{gathered}
\left\langle\Delta n_{3}^{2}\right\rangle_{C}=A R(1-A R)+2 T R^{\prime}, \\
\left\langle\Delta n_{3}^{2}\right\rangle_{F}=A R(1-A R)=\left\langle n_{3}\right\rangle\left(1-\left\langle n_{3}\right\rangle\right), \\
\left\langle\Delta n_{3}^{2}\right\rangle_{B}=A R(1-A R)+4 T R^{\prime} .
\end{gathered}
$$

This shows how the backscattering induces a partition noise $\sim\left\langle n_{3}\right\rangle\left(1-\left\langle n_{3}\right\rangle\right)$, in addition to renormalizing the beamsplitter noise $\sim T R^{\prime}$ via $R^{\prime}=R(1-A)$.

\section{Spin-orbit rotation}

We can incorporate some of the effects of SO-induced rotation within the heuristic scheme presented above. We neglect backscattering for simplicity. Considering the result for the singlet [Eq. (60)] with $\epsilon_{1}=\epsilon_{2}, \quad S_{33}^{S}=\left(e^{2} / h \nu\right) 2 T R(1$ $+\cos \theta_{\mathrm{SO}}$ ), we see that the SO angle $\theta_{\mathrm{SO}}$ "interpolates" from the bosonic behavior $\left(\theta_{\mathrm{SO}}=0\right)$ to the fermionic behavior $\left(\theta_{\mathrm{SO}}=\pi\right)$. For the angle $\theta_{\mathrm{SO}}=\pi / 2$, we actually recover the same result as in the classical case $\left(\epsilon_{1} \neq \epsilon_{2}\right)$-although one must emphasize that any locally rotated state is still maximally entangled, and therefore is not classical. In order to consider particles that effectively behave in an intermediate way between bosons and fermions, we consider particles that follow intermediate statistics, i.e., anyons that acquire a finite phase $e^{i \theta}$ upon antisymmetrization (with $\theta=0$ for bosons and $\theta=\pi$ for fermions). Indeed, one can easily recover the formula

$$
\left\langle\Delta n_{3}^{2}\right\rangle_{A}=2 \operatorname{TR}(1+\cos \theta),
$$

corresponding to a Rashba-rotated singlet pair with $\epsilon_{1}=\epsilon_{2}$ [Eq. (60)] by the following calculation for anyons:

$$
\begin{aligned}
\left\langle n_{3}\right\rangle_{A} & =P(3)+2 P(3,3) \\
& =\left|s_{13} s_{24}+e^{i \theta} s_{14} s_{23}\right|^{2}+2\left|s_{13} s_{23} \frac{1+e^{i \theta}}{\sqrt{2}}\right|^{2} \\
& =R+T=1 \\
\left\langle n_{3}^{2}\right\rangle_{A} & =P(3)+4 P(3,3) \\
& =\left\langle n_{3}^{2}\right\rangle_{A}+4\left|s_{13} s_{23} \frac{1+e^{i \theta}}{\sqrt{2}}\right|^{2} \\
& =1+2 T R(1+\cos \theta) .
\end{aligned}
$$

The factor $\left(1+e^{i \theta}\right) / \sqrt{2}$ interpolates between the Pauli exclusion principle and the bosonic bunching occuring for two particles in the same lead 3 .

\section{APPENDIX D: EVALUATION OF THE NOISE MATRIX ELEMENT}

Here we sketch the derivation of general formulas for the relevant matrix elements in the noise calculation for injected electron pairs. From the noise definition [Eq. (46)] it is clear that we need to evaluate objets of the form 


$$
\begin{aligned}
D= & \langle 0| a_{\mu, \sigma_{\mu}}\left(\varepsilon_{\mu}\right) a_{\nu, \sigma_{\nu}}\left(\varepsilon_{\nu}\right) a_{\alpha, \sigma}^{\dagger}(\varepsilon) \\
& \times a_{\beta, \sigma^{\prime}}\left(\varepsilon^{\prime}\right) a_{\gamma, \sigma_{\gamma}}^{\dagger}\left(\varepsilon_{\gamma}\right) a_{\eta, \sigma_{\eta}}^{\dagger}\left(\varepsilon_{\eta}\right)|0\rangle
\end{aligned}
$$

and

$$
\begin{aligned}
Q= & \langle 0| a_{\mu, \sigma_{\mu}}\left(\varepsilon_{\mu}\right) a_{\nu, \sigma_{\nu}}\left(\varepsilon_{\nu}\right) a_{\alpha, \sigma^{\dagger}}^{\dagger}(\varepsilon) a_{\beta, \sigma^{\prime}}\left(\varepsilon^{\prime}\right) \\
& \times a_{\alpha^{\prime}, \sigma^{\prime \prime}}^{\dagger}\left(\varepsilon^{\prime \prime}\right) a_{\beta^{\prime}, \sigma^{\prime \prime \prime}}\left(\varepsilon^{\prime \prime \prime}\right) a_{\gamma, \sigma_{\gamma}}^{\dagger}\left(\varepsilon_{\gamma}\right) a_{\eta, \sigma_{\eta}}^{\dagger}\left(\varepsilon_{\eta}\right)|0\rangle .
\end{aligned}
$$

Wick's theorem tells us that we can express the above matrix elements in terms of all possible pairings of the fermionic operators. Here the possible nonzero pairings involve only one creation and one destruction operator. For the first case we have

$$
\begin{aligned}
D= & +\left\langle 0\left|a_{\alpha, \sigma}^{\dagger}(\varepsilon) a_{\beta, \sigma^{\prime}}\left(\varepsilon^{\prime}\right)\right| 0\right\rangle\left\langle 0\left|a_{\nu, \sigma_{\nu}}\left(\varepsilon_{\nu}\right) a_{\gamma, \sigma_{\gamma}}^{\dagger}\left(\varepsilon_{\gamma}\right)\right| 0\right\rangle \\
& \times\left\langle 0\left|a_{\mu, \sigma_{\mu}}\left(\varepsilon_{\mu}\right) a_{\eta, \sigma_{\eta}}^{\dagger}\left(\varepsilon_{\eta}\right)\right| 0\right\rangle-\left\langle 0\left|a_{\alpha, \sigma^{\dagger}}^{\dagger}(\varepsilon) a_{\beta, \sigma^{\prime}}\left(\varepsilon^{\prime}\right)\right| 0\right\rangle \\
& \times\left\langle 0\left|a_{\nu, \sigma_{\nu}}\left(\varepsilon_{\nu}\right) a_{\eta, \sigma_{\eta}}^{\dagger}\left(\varepsilon_{\eta}\right)\right| 0\right\rangle\left\langle 0\left|a_{\mu, \sigma_{\mu}}\left(\varepsilon_{\mu}\right) a_{\gamma, \sigma_{\gamma}}^{\dagger}\left(\varepsilon_{\gamma}\right)\right| 0\right\rangle \\
& +\left\langle 0\left|a_{\nu, \sigma_{\nu}}\left(\varepsilon_{\nu}\right) a_{\alpha, \sigma}^{\dagger}(\varepsilon)\right| 0\right\rangle\left\langle 0\left|a_{\beta, \sigma^{\prime}}\left(\varepsilon^{\prime}\right) a_{\gamma, \sigma_{\gamma}}^{\dagger}\left(\varepsilon_{\gamma}\right)\right| 0\right\rangle
\end{aligned}
$$

$$
\begin{aligned}
& \times\left\langle 0\left|a_{\mu, \sigma_{\mu}}\left(\varepsilon_{\mu}\right) a_{\eta, \sigma_{\eta}}^{\dagger}\left(\varepsilon_{\eta}\right)\right| 0\right\rangle-\left\langle 0\left|a_{\nu, \sigma_{\nu}}\left(\varepsilon_{\nu}\right) a_{\alpha, \sigma}^{\dagger}(\varepsilon)\right| 0\right\rangle \\
& \times\left\langle 0\left|a_{\beta, \sigma^{\prime}}\left(\varepsilon^{\prime}\right) a_{\eta, \sigma_{\eta}}^{\dagger}\left(\varepsilon_{\eta}\right)\right| 0\right\rangle\left\langle 0\left|a_{\mu, \sigma_{\mu}}\left(\varepsilon_{\mu}\right) a_{\gamma, \sigma_{\gamma}}^{\dagger}\left(\varepsilon_{\gamma}\right)\right| 0\right\rangle \\
& +\left\langle 0\left|a_{\mu, \sigma_{\mu}}\left(\varepsilon_{\mu}\right) a_{\alpha, \sigma}^{\dagger}(\varepsilon)\right| 0\right\rangle\left\langle 0\left|a_{\nu, \sigma_{\nu}}\left(\varepsilon_{\nu}\right) a_{\gamma, \sigma_{\gamma}}^{\dagger}\left(\varepsilon_{\gamma}\right)\right| 0\right\rangle \\
& \times\left\langle 0\left|a_{\beta, \sigma_{z}^{\prime}}\left(\varepsilon^{\prime}\right) a_{\eta, \sigma_{\eta}}^{\dagger}\left(\varepsilon_{\eta}\right)\right| 0\right\rangle-\left\langle 0\left|a_{\mu, \sigma_{\mu}}\left(\varepsilon_{\mu}\right) a_{\alpha, \sigma}^{\dagger}(\varepsilon)\right| 0\right\rangle \\
& \times\left\langle 0\left|a_{\beta, \sigma^{\prime}}\left(\varepsilon^{\prime}\right) a_{\gamma, \sigma_{\gamma}}^{\dagger}\left(\varepsilon_{\gamma}\right)\right| 0\right\rangle\left\langle 0\left|a_{\nu, \sigma_{\nu}}\left(\varepsilon_{\nu}\right) a_{\eta, \sigma_{\eta}}^{\dagger}\left(\varepsilon_{\eta}\right)\right| 0\right\rangle .
\end{aligned}
$$

The first two terms are zero since they both have a destruction operator acting on the vacuum state. Hence, we have

$$
\begin{aligned}
D= & \delta_{\nu \alpha} \delta_{\sigma_{\nu} \sigma} \delta_{\varepsilon_{\nu} \varepsilon}\left(\delta_{\beta \gamma} \delta_{\mu \eta} \delta_{\sigma^{\prime} \sigma_{\gamma}} \delta_{\sigma_{\mu} \sigma_{\eta}} \delta_{\varepsilon^{\prime} \varepsilon_{\gamma}} \delta_{\varepsilon_{\mu} \varepsilon_{\eta}}\right. \\
& \left.-\delta_{\beta \eta} \delta_{\mu \gamma} \delta_{\sigma^{\prime} \sigma_{\eta}} \delta_{\sigma_{\mu} \sigma_{\gamma}} \delta_{\varepsilon^{\prime} \varepsilon_{\eta}} \delta_{\varepsilon_{\mu} \varepsilon_{\gamma}}\right) \\
& +\delta_{\mu \alpha} \delta_{\sigma_{\mu} \sigma} \delta_{\varepsilon_{\mu} \varepsilon}\left(\delta_{\nu \gamma} \delta_{\beta \eta} \delta_{\sigma_{\nu} \sigma_{\gamma}} \delta_{\sigma^{\prime} \sigma_{\eta}} \delta_{\varepsilon_{\nu} \varepsilon_{\gamma}} \delta_{\varepsilon^{\prime} \varepsilon_{\eta}}\right. \\
& \left.-\delta_{\beta \gamma} \delta_{\nu \eta} \delta_{\sigma^{\prime} \sigma_{\gamma}} \delta_{\sigma_{\nu} \sigma_{\eta}} \delta_{\varepsilon^{\prime} \varepsilon_{\gamma}} \delta_{\varepsilon_{\nu} \varepsilon_{\eta}}\right) .
\end{aligned}
$$

Similarly, we find

$$
\begin{aligned}
& Q=+\delta_{\alpha \beta} \delta_{\alpha^{\prime} \beta^{\prime}} \delta_{\sigma \sigma^{\prime}} \delta_{\sigma^{\prime \prime} \sigma^{\prime \prime \prime}} \delta_{\varepsilon \varepsilon^{\prime}} \delta_{\varepsilon^{\prime \prime} \varepsilon^{\prime \prime \prime}}\left[\delta_{\nu \gamma} \delta_{\mu \eta} \delta_{\sigma_{\nu} \sigma_{\gamma}} \delta_{\sigma_{\mu} \sigma_{\eta}} \delta_{\varepsilon_{\nu^{\varepsilon}} \varepsilon_{\gamma}} \delta_{\varepsilon_{\mu} \varepsilon_{\eta}}-\delta_{\mu \gamma} \delta_{\nu \eta} \delta_{\sigma_{\mu} \sigma_{\gamma}} \delta_{\sigma_{\nu} \sigma_{\eta}} \delta_{\varepsilon_{\mu} \varepsilon_{\gamma}} \delta_{\varepsilon_{\nu^{\varepsilon}} \varepsilon^{\prime}}\right] \\
& -\delta_{\alpha^{\prime} \beta^{\prime}} \delta_{\mu \eta} \delta_{\sigma^{\prime \prime} \sigma^{\prime \prime \prime}} \delta_{\sigma_{\mu} \sigma_{\eta}} \delta_{\varepsilon^{\prime \prime} \varepsilon^{\prime \prime \prime}} \delta_{\varepsilon_{\mu} \eta_{\eta}}\left[\delta_{\nu \gamma} \delta_{\beta \alpha} \delta_{\sigma_{\nu} \sigma_{\gamma}} \delta_{\sigma^{\prime} \sigma^{\prime}} \delta_{\varepsilon_{\nu} \varepsilon_{\gamma}} \delta_{\varepsilon^{\prime} \varepsilon}-\delta_{\nu \alpha} \delta_{\beta \gamma} \delta_{\sigma_{\nu} \sigma} \delta_{\sigma^{\prime} \sigma_{\gamma}} \delta_{\varepsilon_{\nu} \varepsilon} \delta_{\varepsilon^{\prime} \varepsilon_{\gamma}}\right] \\
& +\delta_{\alpha^{\prime} \beta^{\prime}} \delta_{\mu \gamma} \delta_{\sigma^{\prime \prime} \sigma^{\prime \prime \prime}} \delta_{\sigma_{\mu} \sigma_{\gamma}} \delta_{\varepsilon^{\prime \prime} \varepsilon^{\prime \prime \prime}} \delta_{\varepsilon_{\mu} \varepsilon}\left[\delta_{\nu \eta} \delta_{\beta \alpha} \delta_{\sigma_{\nu} \sigma_{\eta}} \delta_{\sigma^{\prime} \sigma^{\prime}} \delta_{\varepsilon_{\nu} \varepsilon_{\eta}} \delta_{\varepsilon^{\prime} \varepsilon}-\delta_{\nu \alpha} \delta_{\beta \eta} \delta_{\sigma_{\nu} \sigma} \delta_{\sigma^{\prime} \sigma_{\eta}} \delta_{\varepsilon_{\nu} \varepsilon} \delta_{\varepsilon^{\prime} \varepsilon_{\eta}}\right] \\
& -\delta_{\alpha^{\prime} \beta^{\prime}} \delta_{\mu \alpha} \delta_{\sigma^{\prime \prime} \sigma^{\prime \prime \prime}} \delta_{\sigma_{\mu} \sigma} \delta_{\varepsilon^{\prime \prime} \varepsilon^{\prime \prime \prime}} \delta_{\varepsilon_{\mu} \varepsilon}\left[\delta_{\nu \eta} \delta_{\beta \gamma} \delta_{\sigma_{\nu} \sigma_{\eta}} \delta_{\sigma^{\prime} \sigma_{\gamma}} \delta_{\varepsilon_{\nu^{\varepsilon}} \varepsilon_{\eta}} \delta_{\varepsilon^{\prime} \varepsilon_{\gamma}}-\delta_{\nu \gamma} \delta_{\beta \eta} \delta_{\sigma_{\nu} \sigma_{\gamma}} \delta_{\sigma^{\prime} \sigma_{\eta}} \delta_{\varepsilon_{\nu} \varepsilon_{\gamma}} \delta_{\varepsilon^{\prime} \varepsilon_{\eta}}\right] \\
& -\delta_{\nu \alpha} \delta_{\mu \eta} \delta_{\sigma_{\nu} \sigma} \delta_{\sigma_{\mu} \sigma_{\eta}} \delta_{\varepsilon_{\nu} \varepsilon} \delta_{\varepsilon_{\mu} \varepsilon}\left[\delta_{\beta \gamma} \delta_{\beta^{\prime} \alpha^{\prime}} \delta_{\sigma^{\prime} \sigma_{\gamma}} \delta_{\sigma^{\prime \prime \prime} \sigma^{\prime \prime}} \delta_{\varepsilon^{\prime} \varepsilon_{\gamma}} \delta_{\varepsilon^{\prime \prime \prime} \varepsilon^{\prime \prime}}-\delta_{\beta \alpha^{\prime}} \delta_{\beta^{\prime} \gamma} \delta_{\sigma^{\prime} \sigma^{\prime \prime}} \delta_{\sigma^{\prime \prime \prime} \sigma_{\gamma}} \delta_{\varepsilon^{\prime} \varepsilon^{\prime \prime}} \delta_{\varepsilon^{\prime \prime \prime} \varepsilon_{\gamma}}\right] \\
& +\delta_{\nu \alpha} \delta_{\mu \gamma} \delta_{\sigma_{\nu} \sigma} \delta_{\sigma_{\mu} \sigma_{\gamma}} \delta_{\varepsilon_{\nu} \varepsilon^{\varepsilon}} \delta_{\varepsilon_{\mu} \varepsilon}\left[\delta_{\beta \eta} \delta_{\beta^{\prime} \alpha^{\prime}} \delta_{\sigma^{\prime} \sigma_{\eta}} \delta_{\sigma^{\prime \prime \prime} \sigma^{\prime \prime}} \delta_{\varepsilon^{\prime} \varepsilon_{\eta}} \delta_{\varepsilon^{\prime \prime \prime} \varepsilon^{\prime \prime}}-\delta_{\beta \alpha^{\prime}} \delta_{\sigma^{\prime} \sigma^{\prime \prime}} \delta_{\varepsilon^{\prime} \varepsilon^{\prime \prime}} \delta_{\beta^{\prime} \eta} \delta_{\sigma^{\prime \prime \prime} \sigma_{\eta}} \delta_{\varepsilon^{\prime \prime \prime} \varepsilon_{\eta}}\right] \\
& -\delta_{\nu \alpha} \delta_{\mu \alpha^{\prime}} \delta_{\sigma_{\nu} \sigma} \delta_{\sigma_{\mu} \sigma^{\prime \prime}} \delta_{\varepsilon_{\nu} \varepsilon} \delta_{\varepsilon_{\mu} \varepsilon^{\varepsilon^{\prime}}}\left[\delta_{\beta \eta} \delta_{\beta^{\prime} \gamma} \delta_{\sigma^{\prime} \sigma_{\eta}} \delta_{\sigma^{\prime \prime \prime} \sigma_{\gamma}} \delta_{\varepsilon^{\prime} \varepsilon_{\eta}} \delta_{\varepsilon^{\prime \prime \prime} \varepsilon_{\gamma}}-\delta_{\beta \gamma} \delta_{\beta^{\prime} \eta} \delta_{\sigma^{\prime} \sigma_{\gamma}} \delta_{\sigma^{\prime \prime \prime} \sigma_{\eta}} \delta_{\varepsilon^{\prime} \varepsilon_{\gamma}} \delta_{\varepsilon^{\prime \prime \prime} \varepsilon_{\eta}}\right] \\
& +\delta_{\mu \alpha} \delta_{\nu \eta} \delta_{\sigma_{\mu} \sigma} \delta_{\sigma_{\nu} \sigma_{\eta}} \delta_{\varepsilon_{\mu} \varepsilon} \delta_{\varepsilon_{\nu} \varepsilon_{\eta}}\left[\delta_{\beta \gamma} \delta_{\sigma^{\prime} \sigma_{\gamma}} \delta_{\varepsilon^{\prime} \varepsilon_{\gamma}} \delta_{\beta^{\prime} \alpha^{\prime}} \delta_{\sigma^{\prime \prime \prime} \sigma^{\prime \prime}} \delta_{\varepsilon^{\prime \prime \prime} \varepsilon^{\prime \prime}}-\delta_{\beta \alpha^{\prime}} \delta_{\sigma^{\prime} \sigma^{\prime \prime}} \delta_{\varepsilon^{\prime} \varepsilon^{\prime \prime}} \delta_{\beta^{\prime} \gamma} \delta_{\sigma^{\prime \prime \prime} \sigma_{\gamma}} \delta_{\varepsilon^{\prime \prime \prime} \varepsilon_{\gamma}}\right] \\
& -\delta_{\mu \alpha} \delta_{\nu \gamma} \delta_{\sigma_{\mu} \sigma} \delta_{\sigma_{\nu} \sigma_{\gamma}} \delta_{\varepsilon_{\mu} \varepsilon} \delta_{\varepsilon_{\nu} \varepsilon}\left[\delta_{\beta \eta} \delta_{\sigma^{\prime} \sigma_{\eta}} \delta_{\varepsilon^{\prime} \varepsilon_{\eta}} \delta_{\beta^{\prime} \alpha^{\prime}} \delta_{\sigma^{\prime \prime \prime} \sigma^{\prime \prime}} \delta_{\varepsilon^{\prime \prime \prime} \varepsilon^{\prime \prime}}-\delta_{\beta \alpha^{\prime}} \delta_{\sigma^{\prime} \sigma^{\prime \prime}} \delta_{\varepsilon^{\prime} \varepsilon^{\prime \prime}} \delta_{\beta^{\prime} \eta} \delta_{\sigma^{\prime \prime \prime} \sigma_{\eta}} \delta_{\varepsilon^{\prime \prime \prime} \varepsilon_{\eta}}\right] \\
& +\delta_{\mu \alpha} \delta_{\nu \alpha^{\prime}} \delta_{\sigma_{\mu} \sigma} \delta_{\sigma_{\nu} \sigma^{\prime \prime}} \delta_{\varepsilon_{\mu} \varepsilon} \delta_{\varepsilon_{\nu^{\prime}} \prime^{\prime \prime}}\left[\delta_{\beta \eta} \delta_{\sigma^{\prime} \sigma_{\eta}} \delta_{\varepsilon^{\prime} \varepsilon_{\eta}} \delta_{\beta^{\prime} \gamma} \delta_{\sigma^{\prime \prime \prime} \sigma_{\gamma}} \delta_{\varepsilon^{\prime \prime \prime} \varepsilon_{\gamma}}-\delta_{\beta \gamma} \delta_{\sigma^{\prime} \sigma_{\gamma}} \delta_{\varepsilon^{\prime} \varepsilon_{\gamma}} \delta_{\beta^{\prime} \eta} \delta_{\sigma^{\prime \prime \prime} \sigma_{\eta}} \delta_{\varepsilon^{\prime \prime \prime} \varepsilon_{\eta}}\right] \text {. }
\end{aligned}
$$

With the help of Eqs. (D4) and (D5) we can systematically determine all the relevant matrix elements appearing in the noise calculation for a particular type of injected electron pair (singlet, triplets, Bell states, etc.).

\section{APPENDIX E: DISCRETE SUMS FOR $h\left(\varepsilon_{1}, \varepsilon_{2}\right)$}

We choose $\varepsilon_{1}$ as our reference energy and define $\Delta=\varepsilon_{2}-\varepsilon_{1}$. We further assume an equidistant discrete single-particle spectrum of the leads, $\varepsilon=\varepsilon_{1}+(q+n) \delta$, where $n$ is an integer, $q$ a real number between 0 and 1 , and $\delta$ is the level spacing of the leads, see Fig. 6. We obtain $h(q)=g_{0}(q-\Delta / \delta) g_{0}(q) h_{0}(q)$ with the discrete sums

$h_{0}(q)=\sum_{n=-\infty}^{\infty} \frac{1}{(n \delta+q \delta-\Delta+i \gamma)(n \delta+q \delta-i \gamma)}=\frac{-2 \pi}{\delta(\Delta-2 i \gamma)} \frac{\sin \pi\left(q+i \frac{\gamma}{\delta}\right)\left[\cos \pi\left(q-3 i \frac{\gamma}{\delta}\right)-\cos \pi\left(q+i \frac{\gamma}{\delta}-2 \frac{\Delta}{\delta}\right)\right]}{\left(\cosh 2 \pi \frac{\gamma}{\delta}-\cos 2 \pi q\right)\left[\cosh 2 \pi \frac{\gamma}{\delta}-\cos 2 \pi\left(q-\frac{\Delta}{\delta}\right)\right]}$, 


$$
\frac{1}{g_{0}(q)^{2}}=\sum_{n=-\infty}^{\infty} \frac{1}{(n \delta+q \delta)^{2}+\gamma^{2}}=\frac{\pi}{\gamma \delta} \frac{\sinh 2 \pi \frac{\gamma}{\delta}}{\cosh 2 \pi \frac{\gamma}{\delta}-\cos 2 \pi q}
$$

Taking the modulus squared of the complex function $h$, we obtain $|h(q)|^{2}=g_{0}(q)^{2} g_{0}(q-\Delta / \delta)^{2}\left|h_{0}(q)\right|^{2}=A B(q)$, with a manifestly $q$-independent factor

$$
A=\frac{\gamma^{2}}{(\Delta / 2)^{2}+\gamma^{2}} \frac{1}{2 \sinh ^{2} 2 \pi \gamma / \delta},
$$

and an apparently $q$-dependent factor

$$
B(q)=\frac{|\cos \pi(q-3 i \gamma / \delta)-\cos \pi(q-2 \Delta / \delta+i \gamma / \delta)|^{2}}{\cosh 2 \pi \gamma / \delta-\cos 2 \pi(q-\Delta / \delta)} .
$$

Inspection of Eq. (E4) shows that the dependence of $B(q)$ on the offset $q$ drops out completely, and we are left with $B \equiv B(q)=\cosh (4 \pi \gamma / \delta)-\cos (2 \pi \Delta / \delta)=\cosh ^{2}(2 \pi \gamma / \delta)$ $-\cos ^{2}(\pi \Delta / \delta)$, where we have used $\cos ^{2} x=(1+\cos x) / 2$ and $\cosh ^{2} x=(1+\cosh x) / 2$. Combining $A$ and $B$, we finally obtain Eq. (131). We note that the cancellation of $q$ from the interference function $|h|^{2}$ is a consequence of our normalization condition, i.e., that electrons are injected with unit probability.
${ }^{1}$ Semiconductor Spintronics and Quantum Computation, edited by D. D. Awschalom, D. Loss, and N. Samarth (Springer, Berlin, 2002).

${ }^{2}$ I. Zutic, J. Fabian, and S. Das Sarma, Rev. Mod. Phys. 76, 323 (2004).

${ }^{3}$ R. C. Liu, B. Odom, Y. Yamamoto, and S. Tarucha, Nature (London) 391, 263 (1998).

${ }^{4}$ J. C. Egues, P. Recher, D. S. Saraga, V. N. Golovach, G. Burkard, E. V. Sukhorukov, and D. Loss, in Quantum Noise in Mesoscopic Physics, edited by Y. V. Nazarov (Kluwer, Dordrecht, The Netherlands, 2003), p. 241-274.

${ }^{5}$ D. P. DiVincenzo and D. Loss, J. Magn. Magn. Mater. 200, 202 (1999).

${ }^{6}$ G. Burkard, D. Loss, and E. V. Sukhorukov, Phys. Rev. B 61, R16303 (2000).

${ }^{7}$ M. S. Choi, C. Bruder, and D. Loss, Phys. Rev. B 62, 13569 (2000).

${ }^{8}$ P. Recher, E. V. Sukhorukov, and D. Loss, Phys. Rev. B 63, 165314 (2001).

${ }^{9}$ G. B. Lesovik, T. Martin, and G. Blatter, Eur. Phys. J. B 24, 287 (2001).

${ }^{10}$ G. Falci, D. Feinberg, and F. W. J. Hekking, Europhys. Lett. 54, 255 (2001).

${ }^{11} \mathrm{R}$. Mérlin, cond-mat/0105073.

${ }^{12}$ A. T. Costa, Jr. and S. Bose, Phys. Rev. Lett. 87, 277901 (2001).

${ }^{13}$ W. D. Oliver, F. Yamaguchi, and Y. Yamamoto, Phys. Rev. Lett. 88, 037901 (2002).

${ }^{14}$ S. Bose and D. Home, Phys. Rev. Lett. 88, 050401 (2002).

${ }^{15}$ P. Recher and D. Loss, Phys. Rev. B 65, 165327 (2002).

${ }^{16}$ C. Bena, S. Vishveshwara, L. Balents, and M. P. A. Fisher, Phys. Rev. Lett. 89, 037901 (2002).

${ }^{17}$ D. S. Saraga, B. L. Altshuler, D. Loss, and R. M. Westervelt, Phys. Rev. Lett. 92, 246803 (2004)

${ }^{18}$ V. Bouchiat, N. Chtchelkatchev, D. Feinberg, G. B. Lesovik, T. Martin, and J. Torrès, Nanotechnology 14, 77 (2003).

${ }^{19}$ P. Recher and D. Loss, Phys. Rev. Lett. 91, 267003 (2003).

${ }^{20}$ C. W. J. Beenakker, C. Emary, M. Kindermann, and J. L. van Velsen, Phys. Rev. Lett. 91, 147901 (2003).

${ }^{21}$ P. Samuelsson, E. V. Sukhorukov, and M. Büttiker, Phys. Rev. Lett. 91, 157002 (2003).

${ }^{22}$ D. S. Saraga, B. L. Altshuler, D. Loss, and R. M. Westervelt,
Phys. Rev. Lett. 92, 246803 (2004).

${ }^{23}$ P. Samuelsson, E. V. Sukhorukov, and M. Büttiker, Phys. Rev. Lett. 92, 026805 (2004).

${ }^{24}$ O. Sauret, T. Martin, and D. Feinberg, Phys. Rev. B 72, 024544 (2005).

${ }^{25}$ E. Dupont and K. Le Hur, cond-mat/0507282 (unpublished).

${ }^{26}$ F. Taddei and R. Fazio, Phys. Rev. B 65, 075317 (2002); L. Faoro, F. Taddei, and R. Fazio, ibid. 69, 125326 (2004).

${ }^{27}$ E. I. Rashba, Fiz. Tverd. Tela (Leningrad) 2, 1224 (1960) [Sov. Phys. Solid State 2, 1109 (1960)]; Y. A. Bychkov and E. I. Rashba, J. Phys. C 17, 6039 (1984); Yu. A. Bychkov and E. I. Rashba, JETP Lett. 39, 78 (1984).

${ }^{28}$ J. C. Egues, G. Burkard, and D. Loss, Phys. Rev. Lett. 89, 176401 (2002).

${ }^{29}$ S. Datta and B. Das, Appl. Phys. Lett. 56, 665 (1990); see also J. C. Egues, G. Burkard, and D. Loss, ibid. 82, 2658 (2003) for a spin FET proposal with additional spin control due to spin-orbit induced interband coupling. G. Meier, T. Matsuyama, and U. Merkt, Phys. Rev. B 65, 125327 (2002) and C.-M. Hu, J. Nitta, A. Jensen, J. B. Hansen, H. Takayanagi, T. Matsuyama, D. Heitmann, and U. Merkt, J. Appl. Phys. 91, 7251 (2002) describe some experimental efforts towards the Datta-Das transistor. See M. G. Pala, M. Governale, J. König, and U. Zülicke, Europhys. Lett. 65, 850 (2004) for a spin-transistor proposal with holes.

${ }^{30}$ A. V. Moroz and C. H. W. Barnes, Phys. Rev. B 60, 14272 (1999).

${ }^{31}$ F. Mireles and G. Kirczenow, Phys. Rev. B 64, 024426 (2001).

${ }^{32}$ L. W. Molenkamp, G. Schmidt, and G. E. W. Bauer, Phys. Rev. B 64, 121202(R) (2001).

${ }^{33}$ M. H. Larsen, A. M. Lunde, and K. Flensberg, Phys. Rev. B 66, 033304 (2002).

${ }^{34}$ M. Governale and U. Zülicke, Phys. Rev. B 66, 073311 (2002).

${ }^{35}$ P. Středa and P. Šeba, Phys. Rev. Lett. 90, 256601 (2003).

${ }^{36}$ E. A. de Andrada e Silva and G. C. La Rocca, Phys. Rev. B 67, 165318 (2003).

${ }^{37}$ X. F. Wang, Phys. Rev. B 69, 035302 (2004).

${ }^{38}$ R. Winkler, Phys. Rev. B 69, 045317 (2004).

${ }^{39}$ S. Q. Shen, Appl. Phys. Lett. 84, 996 (2004).

${ }^{40}$ E. G. Mishchenko, A. Brataas, and Y. Tserkovnyak, Phys. Rev. B 69, 073305 (2004).

${ }^{41}$ G. Usaj and C. A. Balseiro, Phys. Rev. B 70, 041301(R) (2004). 
${ }^{42}$ C. L. Romano, S. E. Ulloa, and P. I. Tamborenea, Phys. Rev. B 71, 035336 (2005).

${ }^{43}$ J. Knobbe and Th. Schäpers, Phys. Rev. B 71, 035311 (2005).

${ }^{44}$ S. Debald and B. Kramer, Phys. Rev. B 71, 115322 (2005).

${ }^{45}$ Yu-Xian Li, Y. Guo, and Bo-Zang Li, Phys. Rev. B 71, 012406 (2005).

${ }^{46}$ G. Dresselhaus, Phys. Rev. 100, 580 (1955).

${ }^{47}$ The separation of both the Dresselhaus and Rashba contributions has recently been investigated experimentally via photocurrent measurements, see S. D. Ganichev, V. V. Bel'kov, L. E. Golub, E. L. Ivchenko, Petra Schneider, S. Giglberger, J. Eroms, J. De Boeck, G. Borghs, W. Wegscheider, D. Weiss, and W. Prettl, Phys. Rev. Lett. 92, 256601 (2004).

${ }^{48}$ Gate control of the SO coupling in 2DEGs was realized by G. Engels, J. Lange, Th. Schäpers, and H. Lüth, Phys. Rev. B 55, R1958 (1997) and J. Nitta, T. Akazaki, H. Takayanagi, and T. Enoki, Phys. Rev. Lett. 78, 1335 (1997). The relevance of band nonparabolicity effects on the SO coupling is discussed by C.-M. Hu, J. Nitta, T. Akazaki, H. Takayanagai, J. Osaka, P. Pfeffer, and W. Zawadzki, Phys. Rev. B 60, 7736 (1999); see also D. Grundler, Phys. Rev. Lett. 84, 6074 (2000) for electric control with front and back gates. Y. Sato, T. Kita, S. Gozu, and S. Yamada, J. Appl. Phys. 89, 8017 (2001) have reported large values $\left(\sim 3 \times 10^{-11} \mathrm{eV} \mathrm{m}\right)$ for the $\mathrm{SO}$ coupling constant in an InAs-based interface. The Rashba coupling in multimode quantum wires was investigated by Th. Schäpers, J. Knobbe, and V. A. Guzenko, Phys. Rev. B 69, 235323 (2004).

${ }^{49}$ J. Schliemann, J. C. Egues, and D. Loss, Phys. Rev. Lett. 90, 146801 (2003).

${ }^{50}$ R. Fiederling, M. Keim, G. Reuscher, W. Ossau, G. Schmidt, A. Waag, and L. W. Molenkamp, Nature (London) 402, 787 (1999); Y. Ohno, D. K. Young, B. Beschoten, F. Matsukura, H. Ohno, and D. D. Awschalom, ibid. 402, 790 (1999).

${ }^{51}$ See J. C. Egues, Phys. Rev. Lett. 80, 4578 (1998) and J. C. Egues, C. Gould, G. Richter, and L. W. Molenkamp, Phys. Rev. B 64, 195319 (2001) for ballistic spin filtering in semimagnetic heterostructures.

${ }^{52}$ For a study of spin-dephasing in multimode wires, see: W. Häusler, Phys. Rev. B 70, 115313 (2004) and B. K. Nikolić and S. Souma, ibid. 71, 195328 (2005).

${ }^{53}$ The band offset due to the gate-induced Rashba term arises from the energy shift $\epsilon_{R} \equiv \hbar^{2} k_{R}^{2} / 2 m$ and is very small since $\epsilon_{R} \ll \varepsilon_{F}$. We can estimate the transmission amplitude from $t=2(1$ $\left.+\epsilon_{R} / \varepsilon_{F}\right)^{1 / 4} /\left[1+\left(1+\epsilon_{R} / \varepsilon_{F}\right)^{1 / 2}\right]$ (see Refs. 32 and 33). Typically (Ref. 48) $\epsilon_{R} / \varepsilon_{F}=0.1$, which yields $|t|^{2}=0.99943$.

${ }^{54}$ N. W. Ashcroft and N. D. Mermin, Solid State Physics (Holt-
Saunders International, New York, 1976), Chap. 9.

${ }^{55}$ See Molenkamp, Schmidt, and Bauer (Ref. 32) and U. Zulicke and C. Schrol, Phys. Rev. Lett. 88, 029701 (2001) for a discussion of the relevance of the proper matching of the velocity operator for transport across hybrid ferromagnetic/ semiconductor junctions. E. A. de Andrada e Silva, G. C. La Rocca, and F. Bassani, Phys. Rev. B 55, 16293 (1997), discuss boundary conditions for the confinement direction in SO coupled systems.

${ }^{56}$ G. Lommer, F. Malcher, and U. Rössler, Phys. Rev. Lett. 60, 728 (1988).

${ }^{57}$ B. Jusserand, D. Richards, H. Peric, and B. Etienne, Phys. Rev. Lett. 69, 848 (1992).

${ }^{58}$ B. Jusserand, D. Richards, G. Allan, C. Priester, and B. Etienne, Phys. Rev. B 51, R4707 (1995).

${ }^{59}$ M. Büttiker, Phys. Rev. B 46, 12485 (1992); Ya. M. Blanter and M. Büttiker, Phys. Rep. 336, 1 (2000). See also G. Feve, W. D. Oliver, M. Aranzana, and Y. Yamamoto, Phys. Rev. B 66, 155328 (2002) for a formulation of the scattering formalism in terms of the Rashba states for a beam-splitter configuration with "global" spin orbit and single-moded incoming leads.

${ }^{60}$ H. Zhao, X. Zhao, and Y.-Q. Li, cond-mat/0502390 (unpublished).

${ }^{61}$ P. Samuelsson, E. V. Sukhorukov, and M. Büttiker, Phys. Rev. B 70, 115330 (2004).

${ }^{62}$ X. Hu and S. Das Sarma, Phys. Rev. B 69, 115312 (2004).

${ }^{63}$ It is crucial that subsequent pairs of entangled electrons are well separated in time and space. This requires that the "delay time" $\tau_{d}$ between two electrons within an entangled pair an electron pair is negligible compared to the "transit time" $\tau_{t}$ for an electron to cross the beam-splitter structure. For typical parameters we find ${ }^{4} \tau_{d} \sim 0.6 \mathrm{ps} \ll \tau_{t} \sim 10-100$ ps for the entanglers of Refs. 8 and 17.

${ }^{64}$ For a recent proposal on how to perform nondestructive projective measurements on Bell states using quantum dots, relevant for measurement-based quantum computation, see: H. A. Engel and D. Loss, Science 309, 586 (2005) and J. Carlos Egues, ibid. 309, 565 (2005) (Perspectives).

${ }^{65}$ R. Loudon, Phys. Rev. A 58, 4904 (1998).

${ }^{66}$ A. I. Signal and U. Zülicke, cond-mat/0505049 (unpublished).

${ }^{67}$ G. Burkard and D. Loss, Phys. Rev. Lett. 91, 087903 (2003).

${ }^{68}$ S. Datta, Electronic Transport in Mesoscopic Systems (Cambridge University Press, Cambridge, 1995).

${ }^{69}$ G. Burkard, J. C. Egues, and D. Loss, J. Supercond. 16, 237 (2003). 